The Astrophysical Journal Supplement Series, 35:359-393, 1977 November

(C) 1977. The American Astronomical Society. All rights reserved. Printed in U.S.A.

\title{
OPTICAL IDENTIFICATION OF 664 OHIO SOURCES USING ACCURATE RADIO AND OPTICAL POSITIONS MEASURED BY THE TEXAS INTERFEROMETERS
}

\author{
FRANK D. GHIGo \\ The University of Texas at Austin \\ Received 1977 April 4; accepted 1977 May 23
}

\begin{abstract}
Results of optical identification work are reported for 664 radio sources selected from the Ohio $1415 \mathrm{MHz}$ survey. Radio positions were measured at $365 \mathrm{MHz}$ to an accuracy of about 2" with the Texas broad-band synthesis interferometer. Forty previously unpublished radio positions are given. Optical positions of 0.5 to 0".7 accuracy were measured on the Palomar Sky Survey (PSS) plates with a two-coordinate laser-interferometer measuring machine. These errors are consistent with those derived by comparing the positions with other accurate optical positions. Background objects were counted on the PSS plates and were used along with counts of objects within $20^{\prime \prime}$ of the radio sources to estimate the radial distribution of the radio-optical offsets of the true identifications. This analysis showed that about $30 \%$ of the identifications have radio-optical separations in excess of those expected from the position errors. This effect appears to be primarily due to resolution of large-angular-size sources by the interferometer system. The completeness and reliability of the identifications are discussed. A total of 319 identifications are suggested. Galaxies and probable galaxies with $m_{v} \leq 19.5$ number $102(15 \%)$. Quasars, probable quasars, and BL Lacertae-type objects with $m_{v} \leq 19.5$ number $136(20 \%)$. One hundred eighteen new or revised identifications are found. Thirteen identification candidates, found to have stellar spectra, are probably random coincidences. Two probable BL Lacertae-type objects $(2207+020$ and $2217+$ 018) appear to have steep radio spectra.
\end{abstract}

Subject headings: galaxies: general — quasars — radio sources: general

\section{INTRODUCTION}

The Ohio radio source survey (Rinsland, Dixon, and Kraus 1975 , and references therein) covers virtually the whole area between declinations $-36^{\circ}$ and $+63^{\circ}$, and contains almost 20,000 sources, making it by far the most extensive survey at $1415 \mathrm{MHz}$ or any higher frequency. Clearly, a completely identified Ohio survey would be of great value. Unfortunately, its position uncertainties $\left(1^{\prime}\right.$ to $\left.10^{\prime}\right)$ are too great to permit reliable identifications, and its flux densities, for sources fainter than $1 \mathrm{Jy},{ }^{1}$ are too uncertain $(\geqslant 50 \%$; Jauncey and Niell 1971; Bridle et al. 1972) for statistically useful samples to be defined. The present work is a step toward alleviating the first problem by measuring positions accurately. The sample presented here is a representative selection of Ohio sources in three areas of sky covering 0.842 steradians. These data will be suitable for statistical studies of quasars and radio galaxies when accurate $1415 \mathrm{MHz}$ flux densities become available for the Ohio sources in these regions.

The selection effects in this sample are discussed in $\S$ II. The Texas radio observations are summarized in $\S$ III. In $\S I V$ the Texas and Ohio positions are compared.

The optical measurements are described in $\S \mathrm{V}$, in which the table of optical data is also found. Comments on individual objects are in $\S \mathrm{Vg}$. In $\S \mathrm{VI}$, the

\footnotetext{
${ }^{1} 1$ Jansky $(J y)=10^{-26} \mathrm{~W} \mathrm{~m}^{-2} \mathrm{~Hz}^{-1}$.
}

Texas optical positions are compared with other accurate optical positions. The results of counting background objects on the PSS plates are given in $\S$ VII. The effect of resolved sources and the completeness and reliability of the sample are discussed in $\S$ VIII.

The identification content of the sample is summarized in $\S$ IX. A discussion of the neutral stellar object (NSO) identifications and the possibility of radio stars in the sample is given. Among the NSOs, two probable BL Lacertae types appear to have steep radio spectra, although these objects more typically have flat spectra.

\section{SELECTION EFFECTS IN THE SAMPLE}

The regions of sky observed are the three areas listed in Table 1. Objects in these regions were scheduled for observing in accordance with the requirements of the broad-band synthesis interferometer (BSI) of the University of Texas Radio Astronomy Observatory (UTRAO), which takes data on the meridian. The instrument is discussed by Douglas et al. (1973). A 3 minute observation of each source was made, so no more than about 200 sources could be observed in a single night. A complete observing pass is five nights of observation, to allow scans to be made on all eight interferometer baselines. Five passes were made through each of the three regions. Eighty percent of the Ohio sources could be scheduled in five 
TABLE 1

SUMmaRy OF RADIO OBSERVATIONS

\begin{tabular}{|c|c|c|c|}
\hline & \multicolumn{3}{|c|}{ REGION } \\
\hline & 1 & 2 & 3 \\
\hline $\begin{array}{l}\text { Right Ascension } \ldots \ldots \ldots \ldots \ldots \ldots \\
\text { Declination } \ldots \ldots \ldots \ldots \ldots \ldots \\
\text { Dates of Observations } \ldots \ldots \ldots \ldots \\
\text { Area (steradians) } \ldots \ldots \ldots \ldots \ldots \ldots\end{array}$ & $\begin{array}{l}8^{\mathrm{h}} \text { to } 15^{\mathrm{h}} \\
23^{\circ} \text { to } 33^{\circ} \\
1971 \text { March-April } \\
0.282\end{array}$ & $\begin{array}{l}12^{\mathrm{h}} \text { to } 18{ }^{\mathrm{h}} 5 \\
10^{\circ} \text { to } 20^{\circ} \\
1971 \text { May } \\
0.287\end{array}$ & $\begin{array}{l}20 \mathrm{~h} .5 \text { to } 1 \frac{\mathrm{h} .5}{2^{\circ}} \\
-2^{\circ} \text { to } 10^{\circ} \\
1971 \text { Aug.-Sept. } \\
0.273\end{array}$ \\
\hline
\end{tabular}

passes. The remaining $20 \%$ were missed because they were in areas where more than five sources occurred in a 3 minute interval of right ascension.

Incompleteness also occurs because the Ohio survey is confusion-limited. Stull (1973) has found that about $30 \%$ of Ohio sources are blends of two or more sources. About one-third of the blended sources which could be scheduled on the BSI were observed and good positions measured. The remainder were missed because no source could be seen within several arc minutes of the listed Ohio catalog position.

The loss of sources from the final sample because of the scheduling procedure and the effect of Ohio catalog confusion means that sources are preferentially lost from crowded regions. This should not seriously bias the final sample since there appears to be no evidence of radio source clustering (e.g., Webster 1976).

The present sample is also biased by the selection in spectral index (by observing a $1415 \mathrm{MHz}$ survey at $365 \mathrm{MHz}$ ) and in angular size (since the BSI fringe size is about $1^{\prime}$, whereas the Ohio beam is about $\left.10^{\prime} \times 40^{\prime}\right)$. The number of sources that would be missed from a complete sample at $1415 \mathrm{MHz}$ was estimated from the spectral index distribution found for the $1400 \mathrm{MHz}$ NRAO survey (Bridle et al. 1972), and from the distribution of angular sizes in the 3CR catalog (Macdonald, Kenderdine, and Neville 1968; Elsmore and Mackay 1969; Mackay 1969). These estimates show that about $5 \%$ of the Ohio catalog was lost due to the spectral index distribution, and about $13 \%$ were lost because they had angular sizes $\geqslant 1^{\prime}$.

One thousand two hundred forty-seven Ohio sources occur in the selected regions with $S_{1415} \geq$ $0.3 \mathrm{Jy}$. The estimates of combined losses due to scheduling, blending of sources, angular size being too large, and radio spectrum being too flat appear to be adequate to account for our having found only 532 sources having $\gamma S_{365} \geq 0.5 \mathrm{Jy}$ and $S_{1415} \geq 0.3 \mathrm{Jy}$ in the present sample $[\gamma$ is the BSI fringe visibility. The BSI fringe separation is $52^{\prime \prime} \sec (30.1-\delta)$ in declination, and 56".7 in right ascension].

A number of sources with flux densities below the aforementioned limits are included in the data to be presented, bringing the total number of sources listed in this paper to 664 .

\section{RADIO OBSERVATIONS}

The BSI and its data reduction are described by Douglas et al. (1973). The radio positions of most sources in this sample are given by Douglas et al. (1973), Ghigo and Owen (1973), and Sharp and Bash (1975). Twenty-one new positions, 19 corrections of published lobe-shifted positions, and 177 positions improved by averaging positions from two or more BSI observing programs are presented in Table 2 . The median radio position errors for the sample of 664 sources are 2 .2. in right ascension and $1^{\prime \prime} .5$ in declination. The true position errors will be somewhat in excess of the listed errors for resolved sources, which are indicated by having $R$ or $R R$ appended to the lobeshift class, which appears in column (8) of Table 2. For a position $(\alpha, \delta)$, the allowed lobe-shift positions $\left(\alpha^{*}, \delta^{*}\right)$ are given by

$$
\left(\alpha^{*}, \delta^{*}\right)=\left(\alpha+k_{\alpha} 1_{\alpha}, \delta+k_{\delta} 1_{\delta}\right),
$$

where

$$
\begin{aligned}
& 1_{\alpha}=3.782 \sec \delta \\
& 1_{\delta}=52^{\prime \prime} .22 \sec (30.1-\delta)
\end{aligned}
$$

and

$$
\left(k_{\alpha}+k_{\delta}\right)=\text { an even integer . }
$$

The key to Table 2 is as follows:

Column (1): Source name in Parkes notation.

Column (2): Notes regarding the position: \$ means the position is the mean of two or more positions from different BSI observing programs; $L$ means that a lobe-shift position was previously published but is corrected here; $N$ means that no BSI position has previously been published for this source.

Column (3): The 1950.0 right ascension (h, m, s) and its error (s).

Column (4): The 1950.0 declination (degrees, minutes, seconds) and its error (arcsec).

Column (5): $N$ and $M$ are the number of independent observations in right ascension and declinations, respectively. $N=3$ and $M=5$ for a complete, single-pass observation (all eight baselines).

Column (6): Galactic coordinates, $l^{\text {II }}$ and $b^{\text {II }}$ (degrees).

Column (7): Measured flux density $\left(\gamma S_{365}\right.$, where $S_{365}$ is the source's total flux density) and its error, in Jy, at the observed centroid.

Column (8): Lobe-shift and resolution class (these are more fully described in Ghigo and Owen 1973): class 1 -probability of lobe shift less than $1 \%$; classes 
TABLE 2

New and Revised Radio Positions From the TeXas INTERferometer

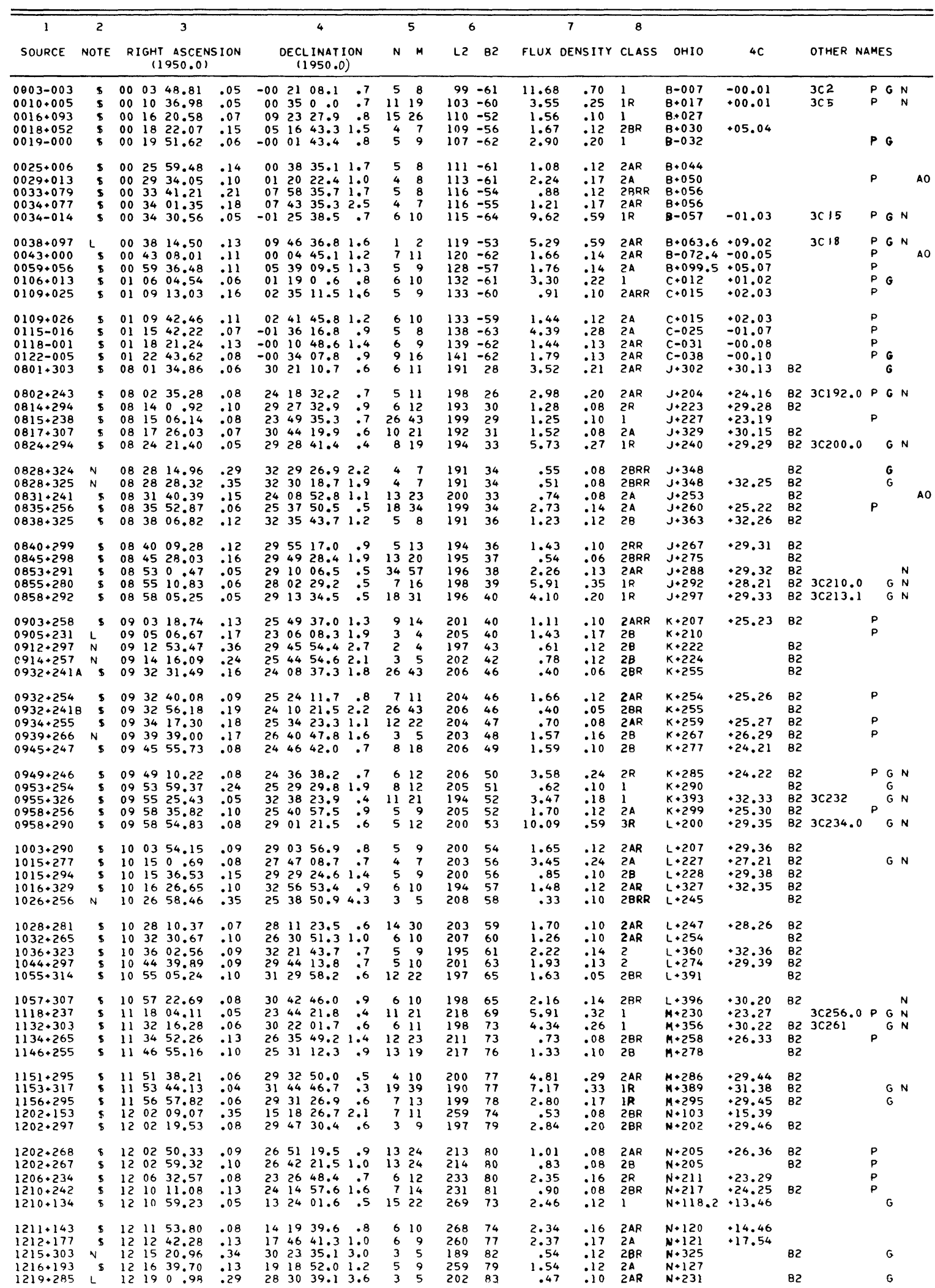


TABLE 2-Continued

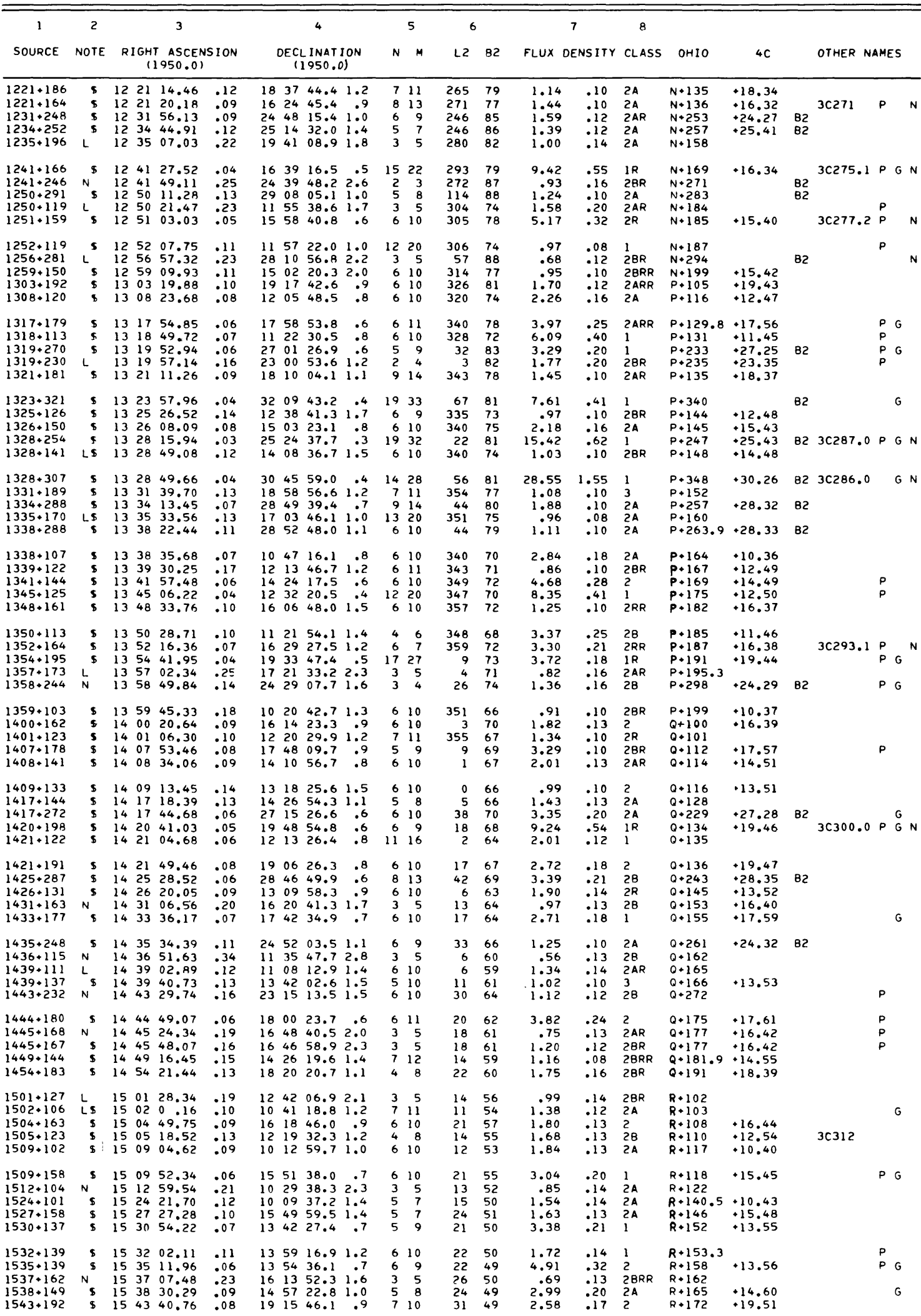


TABLE 2-Continued

\begin{tabular}{|c|c|c|c|c|c|c|c|c|c|c|c|c|c|c|c|c|c|c|c|c|c|}
\hline \multirow{3}{*}{$\begin{array}{c}1 \\
\text { SOURCE } \\
1544+118 \\
1551+179 \\
1554+165 \\
1602+178 \\
1616+138\end{array}$} & \multirow{3}{*}{$\begin{array}{c}2 \\
\text { Note } \\
{ }_{5}^{5} \\
{ }_{5}^{5}\end{array}$} & \multirow{2}{*}{\multicolumn{4}{|c|}{$\begin{array}{c}3 \\
\text { RIGHT ASCENSION } \\
(1950.0)\end{array}$}} & \multirow{2}{*}{\multicolumn{4}{|c|}{$\begin{array}{c}4 \\
\text { DECLINATION } \\
(1950.0) \\
\end{array}$}} & \multicolumn{2}{|r|}{5} & \multicolumn{2}{|c|}{6} & \multicolumn{2}{|r|}{7} & \multirow{2}{*}{$\begin{array}{c}8 \\
\text { CLASS }\end{array}$} & \multirow[b]{2}{*}{ OHIO } & \multirow[b]{2}{*}{$4 C$} & \multirow{2}{*}{\multicolumn{2}{|c|}{ OTHER NAME }} & \multirow[b]{2}{*}{ AES } \\
\hline & & & & & & & & & & N & M & L2 & 82 & FLUX 0 & DENSITY & & & & & & \\
\hline & & $\begin{array}{l}15 \\
15 \\
15 \\
16 \\
16\end{array}$ & $\begin{array}{l}44 \\
51 \\
54 \\
02 \\
16\end{array}$ & $\begin{array}{l}48.57 \\
03.94 \\
34.00 \\
54.21 \\
42.70\end{array}$ & $\begin{array}{l}.25 \\
.13 \\
.44 \\
.14 \\
.07\end{array}$ & $\begin{array}{l}11 \\
17 \\
16 \\
17 \\
13\end{array}$ & $\begin{array}{l}52 \\
58 \\
32 \\
51 \\
48\end{array}$ & $\begin{array}{l}53.6 \\
34.2 \\
28.2 \\
47.9 \\
42.9\end{array}$ & $\begin{array}{l}2.9 \\
1.5 \\
3.3 \\
2.0 \\
.8\end{array}$ & $\begin{array}{l}3 \\
8 \\
3 \\
6 \\
6\end{array}$ & $\begin{array}{r}5 \\
12 \\
5 \\
8 \\
10\end{array}$ & $\begin{array}{l}21 \\
30 \\
29 \\
31 \\
28\end{array}$ & $\begin{array}{l}46 \\
47 \\
46 \\
44 \\
40\end{array}$ & $\begin{array}{r}.80 \\
1.12 \\
.52 \\
1.18 \\
2.55\end{array}$ & $\begin{array}{l}.14 \\
.10 \\
.13 \\
.12 \\
.17\end{array}$ & $\begin{array}{l}2 B R \\
2 B R \\
2 B R R \\
2 A R \\
2 A\end{array}$ & $\begin{array}{l}R+174 \\
R+185.5 \\
R+191 \\
S+106 \\
S+130\end{array}$ & $\begin{array}{r}+17.64 \\
+17.66 \\
+13.58\end{array}$ & & & \\
\hline $\begin{array}{l}1621+132 \\
1641+173 \\
1645+174 \\
1650+111 \\
1653+163\end{array}$ & $L^{L} N^{5}$ & $\begin{array}{l}16 \\
16 \\
16 \\
16 \\
16\end{array}$ & $\begin{array}{l}21 \\
41 \\
45 \\
50 \\
53\end{array}$ & $\begin{array}{l}11.78 \\
34.69 \\
28.03 \\
41.11 \\
29.36\end{array}$ & $\begin{array}{l}.15 \\
.05 \\
.05 \\
.52 \\
.31\end{array}$ & $\begin{array}{l}13 \\
17 \\
17 \\
11 \\
16\end{array}$ & $\begin{array}{l}13 \\
21 \\
25 \\
10 \\
18\end{array}$ & $\begin{array}{l}02.8 \\
21.1 \\
27.7 \\
46.7 \\
40.7\end{array}$ & $\begin{array}{r}1.4 \\
.6 \\
.5 \\
3.0 \\
2.1\end{array}$ & $\begin{array}{r}3 \\
12 \\
24 \\
2 \\
3\end{array}$ & $\begin{array}{r}5 \\
19 \\
40 \\
4 \\
5\end{array}$ & $\begin{array}{l}28 \\
35 \\
36 \\
29 \\
35\end{array}$ & $\begin{array}{l}39 \\
36 \\
35 \\
31 \\
33\end{array}$ & $\begin{array}{l}1.56 \\
9.23 \\
3.77 \\
.53 \\
.78\end{array}$ & $\begin{array}{l}.18 \\
.60 \\
.24 \\
.16 \\
.14\end{array}$ & $\begin{array}{l}2 A \\
1 \\
1 \\
2 B R \\
2 B R\end{array}$ & $\begin{array}{l}S+135 \\
S+170 \\
S+176 \\
S+185 \\
S+188\end{array}$ & $\begin{array}{r}+17.70 \\
+17.71\end{array}$ & $3 C 346.0$ & $P$ & $\begin{array}{l}G \\
G N \\
G N\end{array}$ \\
\hline $\begin{array}{l}1707+117 \\
1722+105 \\
1725+167 \\
1727+174 \\
1732+160\end{array}$ & $\begin{array}{l}N_{\text {s }} \\
\text { s } \\
\text { s } \\
\text { S }\end{array}$ & $\begin{array}{l}17 \\
17 \\
17 \\
17 \\
17\end{array}$ & $\begin{array}{l}07 \\
22 \\
25 \\
27 \\
32\end{array}$ & $\begin{array}{l}14.75 \\
19.90 \\
21.67 \\
53.98 \\
28.08\end{array}$ & $\begin{array}{l}.16 \\
.25 \\
.14 \\
.09 \\
.05\end{array}$ & $\begin{array}{l}11 \\
10 \\
16 \\
17 \\
16\end{array}$ & $\begin{array}{l}44 \\
33 \\
46 \\
26 \\
02\end{array}$ & $\begin{array}{l}13.6 \\
33.2 \\
54.2 \\
37.3 \\
27.3\end{array}$ & $\begin{array}{l}2.2 \\
1.4 \\
1.7 \\
1.1 \\
.7\end{array}$ & $\begin{array}{l}3 \\
5 \\
5 \\
6 \\
6\end{array}$ & $\begin{array}{r}5 \\
9 \\
7 \\
10 \\
10\end{array}$ & $\begin{array}{l}32 \\
33 \\
39 \\
40 \\
39\end{array}$ & $\begin{array}{l}28 \\
24 \\
26 \\
25 \\
24\end{array}$ & $\begin{array}{l}1.12 \\
1.22 \\
1.42 \\
1.98 \\
5.54\end{array}$ & $\begin{array}{l}.16 \\
.14 \\
.16 \\
.16 \\
.35\end{array}$ & $\begin{array}{l}2 A R \\
2 A \\
2 A R \\
2 B R \\
1\end{array}$ & $\begin{array}{l}T+112.1 \\
T+136.8 \\
T+143 \\
T+146 \\
T+154\end{array}$ & $\begin{array}{l} \\
+16.48 \\
+17.76 \\
+16.49\end{array}$ & & $P$ & \\
\hline $\begin{array}{l}1739+107 \\
1740+162 \\
1743+173 \\
1744+114 \\
1751+153\end{array}$ & $\begin{array}{l}L^{S} \\
L \\
L\end{array}$ & $\begin{array}{l}17 \\
17 \\
17 \\
17 \\
17\end{array}$ & $\begin{array}{l}39 \\
40 \\
43 \\
44 \\
51\end{array}$ & $\begin{array}{l}20.11 \\
36.46 \\
22.57 \\
36.61 \\
59.45\end{array}$ & $\begin{array}{l}.12 \\
.15 \\
.24 \\
.19 \\
.22\end{array}$ & $\begin{array}{l}10 \\
16 \\
17 \\
11 \\
15\end{array}$ & $\begin{array}{l}45 \\
14 \\
21 \\
28 \\
21\end{array}$ & $\begin{array}{l}40.5 \\
08.5 \\
10.3 \\
22.1 \\
06.6\end{array}$ & $\begin{array}{l}1.6 \\
1.4 \\
2.3 \\
2.0 \\
3.2\end{array}$ & $\begin{array}{l}9 \\
2 \\
3 \\
3 \\
6\end{array}$ & $\begin{array}{r}14 \\
4 \\
5 \\
5 \\
10\end{array}$ & $\begin{array}{l}35 \\
40 \\
42 \\
36 \\
41\end{array}$ & $\begin{array}{l}20 \\
22 \\
22 \\
19 \\
19\end{array}$ & $\begin{array}{r}1.15 \\
1.72 \\
.92 \\
1.08 \\
.49\end{array}$ & $\begin{array}{l}.12 \\
.16 \\
.16 \\
.16 \\
.10\end{array}$ & $\begin{array}{l}2 B R R \\
2 B R \\
2 A \\
2 A R \\
2 B R\end{array}$ & $\begin{array}{l}T+165.7 \\
T+168 \\
T+172 \\
T+175 \\
T+186\end{array}$ & $\begin{array}{r}+10.53 \\
+16.52\end{array}$ & & & $G$ \\
\hline $\begin{array}{l}1754+159 \\
1755+153 \\
1758+111 \\
1759+138 \\
1810+170\end{array}$ & $\begin{array}{l}\mathrm{N} \\
\mathrm{N} \\
\text { S } \\
\text { S } \\
\mathrm{S}\end{array}$ & $\begin{array}{l}17 \\
17 \\
17 \\
17 \\
18\end{array}$ & $\begin{array}{l}54 \\
55 \\
58 \\
59 \\
10\end{array}$ & $\begin{array}{l}19.31 \\
53.56 \\
08.82 \\
21.67 \\
45.43\end{array}$ & $\begin{array}{l}.19 \\
.23 \\
.18 \\
.06 \\
.13\end{array}$ & $\begin{array}{l}15 \\
15 \\
11 \\
13 \\
17\end{array}$ & $\begin{array}{l}54 \\
18 \\
06 \\
51 \\
05\end{array}$ & $\begin{array}{l}06.5 \\
12.0 \\
12.8 \\
22.8 \\
13.8\end{array}$ & $\begin{array}{l}1.9 \\
1.9 \\
1.2 \\
.7 \\
2.0\end{array}$ & $\begin{array}{l}3 \\
5 \\
8 \\
6 \\
7\end{array}$ & $\begin{array}{r}5 \\
9 \\
13 \\
10 \\
11\end{array}$ & $\begin{array}{l}41 \\
41 \\
37 \\
40 \\
44\end{array}$ & $\begin{array}{l}19 \\
18 \\
16 \\
17 \\
16\end{array}$ & $\begin{array}{l}1.02 \\
.74 \\
1.53 \\
7.34 \\
1.03\end{array}$ & $\begin{array}{l}.16 \\
.12 \\
.14 \\
.45 \\
.12\end{array}$ & $\begin{array}{l}2 A \\
2 A \\
2 A \\
1 \\
2 A\end{array}$ & $\begin{array}{l}T+190 \\
T+193 \\
T+197 \\
T+198 \\
U+119\end{array}$ & $\begin{array}{r}+10.54 \\
+13.66 \\
+17.78\end{array}$ & & $P$ & $\mathbf{G}$ \\
\hline $\begin{array}{l}1822+127 \\
2042+045 \\
2045+068 \\
2055+055 \\
2108+039\end{array}$ & $\begin{array}{l}\text { s } \\
\text { s } \\
\text { s } \\
\text { s } \\
\text { s }\end{array}$ & $\begin{array}{l}18 \\
20 \\
20 \\
20 \\
21\end{array}$ & $\begin{array}{l}22 \\
42 \\
45 \\
55 \\
08\end{array}$ & $\begin{array}{l}42.73 \\
15.70 \\
44.35 \\
59.65 \\
40.32\end{array}$ & $\begin{array}{l}.10 \\
.11 \\
.04 \\
.09 \\
.09\end{array}$ & $\begin{array}{l}12 \\
04 \\
06 \\
05 \\
03\end{array}$ & $\begin{array}{l}46 \\
33 \\
50 \\
31 \\
58\end{array}$ & $\begin{array}{l}39.3 \\
09.5 \\
10.8 \\
12.1 \\
42.8\end{array}$ & $\begin{array}{r}1.0 \\
1.2 \\
.5 \\
.9 \\
1.1\end{array}$ & $\begin{array}{r}5 \\
5 \\
17 \\
4 \\
5\end{array}$ & $\begin{array}{r}9 \\
9 \\
30 \\
7 \\
8\end{array}$ & $\begin{array}{l}41 \\
51 \\
54 \\
54 \\
55\end{array}$ & $\begin{array}{r}11 \\
-22 \\
-22 \\
-25 \\
-28\end{array}$ & $\begin{array}{l}1.96 \\
1.65 \\
7.42 \\
2.56 \\
2.68\end{array}$ & $\begin{array}{l}.16 \\
.14 \\
.41 \\
.21 \\
.20\end{array}$ & $\begin{array}{l}2 A \\
2 A \\
1 \\
2 A R \\
2 A\end{array}$ & $\begin{array}{l}u+139 \\
w+071 \\
w+076 \\
w+093 \\
x+014\end{array}$ & $\begin{array}{r}+12.64 \\
+04.71 \\
+06.68 \\
+05.78 \\
+03.50\end{array}$ & 30424.0 & $P$ & G N \\
\hline $\begin{array}{l}2115+056 \\
2120+044 \\
2128+048 \\
2128+089 \\
2134+004\end{array}$ & $\begin{array}{l}5 \\
\text { s } \\
5 \\
5 \\
5\end{array}$ & $\begin{array}{l}21 \\
21 \\
21 \\
21 \\
21\end{array}$ & $\begin{array}{l}15 \\
20 \\
28 \\
28 \\
34\end{array}$ & $\begin{array}{l}11.06 \\
50.79 \\
02.55 \\
54.24 \\
05.06\end{array}$ & $\begin{array}{l}.17 \\
.12 \\
.05 \\
.06 \\
.09\end{array}$ & $\begin{array}{l}05 \\
04 \\
04 \\
08 \\
00\end{array}$ & $\begin{array}{l}39 \\
27 \\
49 \\
59 \\
28\end{array}$ & $\begin{array}{l}35.8 \\
03.4 \\
04.1 \\
19.4 \\
25.8\end{array}$ & $\begin{array}{r}1.6 \\
1.4 \\
.6 \\
.7 \\
1.2\end{array}$ & $\begin{array}{r}5 \\
5 \\
17 \\
8 \\
6\end{array}$ & $\begin{array}{r}8 \\
8 \\
28 \\
13 \\
10\end{array}$ & $\begin{array}{l}57 \\
57 \\
59 \\
63 \\
55\end{array}$ & $\begin{array}{l}-29 \\
-30 \\
-32 \\
-29 \\
-35\end{array}$ & $\begin{array}{l}1.14 \\
1.62 \\
3.43 \\
3.23 \\
1.95\end{array}$ & $\begin{array}{l}.13 \\
.16 \\
.20 \\
.22 \\
.16\end{array}$ & $\begin{array}{l}2 A R \\
2 A \\
1 \\
1 \\
1 R\end{array}$ & $\begin{array}{l}x+027 \\
x+035 \\
x+046 \\
x+049 \\
x+057\end{array}$ & $\begin{array}{l}+05.79 \\
+04.74 \\
+08.62\end{array}$ & & & $\begin{array}{l}\mathbf{G} \\
\mathbf{G} \\
\mathbf{G}\end{array}$ \\
\hline $\begin{array}{l}2142+077 \\
2142+042 \\
2145+067 \\
2150+053 \\
2152+017\end{array}$ & $\begin{array}{l}\text { s } \\
\text { s } \\
\text { s } \\
\text { s } \\
\text { s }\end{array}$ & $\begin{array}{l}21 \\
21 \\
21 \\
21 \\
21\end{array}$ & $\begin{array}{l}42 \\
42 \\
45 \\
50 \\
52\end{array}$ & $\begin{array}{l}39.42 \\
46.99 \\
36.11 \\
54.34 \\
0.95\end{array}$ & $\begin{array}{l}.12 \\
.07 \\
.04 \\
.07 \\
.31\end{array}$ & $\begin{array}{l}07 \\
04 \\
06 \\
05 \\
01\end{array}$ & $\begin{array}{l}46 \\
17 \\
43 \\
22 \\
44\end{array}$ & $\begin{array}{l}31.5 \\
40.7 \\
40.6 \\
08.9 \\
06.7\end{array}$ & $\begin{array}{r}1.2 \\
.8 \\
.4 \\
.8 \\
2.4\end{array}$ & $\begin{array}{r}5 \\
6 \\
22 \\
5 \\
4\end{array}$ & $\begin{array}{r}9 \\
11 \\
37 \\
9 \\
7\end{array}$ & $\begin{array}{l}64 \\
61 \\
64 \\
63 \\
60\end{array}$ & $\begin{array}{l}-33 \\
-35 \\
-34 \\
-36 \\
-38\end{array}$ & $\begin{array}{l}1.46 \\
3.40 \\
3.95 \\
3.80 \\
1.06\end{array}$ & $\begin{array}{l}.13 \\
.24 \\
.18 \\
.25 \\
.12\end{array}$ & $\begin{array}{l}2 A \\
2 A \\
1 \\
2 A \\
2 A R\end{array}$ & $\begin{array}{l}x+071 \\
x+072 \\
x+076.1 \\
x+085 \\
x+089\end{array}$ & $\begin{array}{l}+07.56 \\
+04.75 \\
+06.69 \\
+05.81\end{array}$ & & & $\begin{array}{l}\mathbf{G} \\
\mathbf{G}\end{array}$ \\
\hline $\begin{array}{l}2203+056 \\
2207+020 \\
2209+080 \\
2226+089 \\
2241+013\end{array}$ & $\begin{array}{l}5 \\
5 \\
5 \\
5 \\
5\end{array}$ & $\begin{array}{l}22 \\
22 \\
22 \\
22 \\
22\end{array}$ & $\begin{array}{l}03 \\
07 \\
09 \\
26 \\
41\end{array}$ & $\begin{array}{l}50.25 \\
0.21 \\
32.04 \\
38.34 \\
16.83\end{array}$ & $\begin{array}{l}.08 \\
.06 \\
.06 \\
.05 \\
.09\end{array}$ & $\begin{array}{l}05 \\
02 \\
08 \\
08 \\
01\end{array}$ & $\begin{array}{l}41 \\
03 \\
04 \\
59 \\
20\end{array}$ & $\begin{array}{l}32.4 \\
57.4 \\
25.6 \\
0.02 \\
40.7\end{array}$ & $\begin{array}{r}1.0 \\
.8 \\
.6 \\
.6 \\
.9\end{array}$ & $\begin{array}{r}6 \\
7 \\
6 \\
10 \\
7\end{array}$ & $\begin{array}{l}10 \\
12 \\
11 \\
16 \\
13\end{array}$ & $\begin{array}{l}66 \\
63 \\
70 \\
74 \\
71\end{array}$ & $\begin{array}{l}-38 \\
-41 \\
-37 \\
-40 \\
-48\end{array}$ & $\begin{array}{l}2.51 \\
3.08 \\
4.71 \\
4.04 \\
1.75\end{array}$ & $\begin{array}{l}.18 \\
.20 \\
.30 \\
.21 \\
.13\end{array}$ & $\begin{array}{l}2 A \\
2 A \\
1 \\
1 \\
2 A\end{array}$ & $\begin{array}{l}Y+007 \\
x+011 \\
Y+015.8 \\
y+045 \\
y+069\end{array}$ & $\begin{array}{r}+05.82 \\
+02.54 \\
+08.64 \\
+08.67 \\
+01.72\end{array}$ & & $\begin{array}{l}P \\
P \\
P \\
P\end{array}$ & G \\
\hline $\begin{array}{l}2308+017 \\
2309+090 \\
2314+038 \\
2320+079 \\
2330+015\end{array}$ & $\begin{array}{l}5 \\
5 \\
5 \\
5 \\
5\end{array}$ & $\begin{array}{l}23 \\
23 \\
23 \\
23 \\
23\end{array}$ & $\begin{array}{l}08 \\
09 \\
14 \\
20 \\
30\end{array}$ & $\begin{array}{l}17.55 \\
56.52 \\
02.31 \\
03.95 \\
43.27\end{array}$ & $\begin{array}{l}.12 \\
.05 \\
.04 \\
.07 \\
.11\end{array}$ & $\begin{array}{l}01 \\
09 \\
03 \\
07 \\
01\end{array}$ & $\begin{array}{l}45 \\
03 \\
48 \\
55 \\
33\end{array}$ & $\begin{array}{l}07.2 \\
08.9 \\
54.9 \\
33.4 \\
52.2\end{array}$ & $\begin{array}{r}1.2 \\
.6 \\
.6 \\
.8 \\
1.2\end{array}$ & $\begin{array}{r}5 \\
8 \\
11 \\
17 \\
5\end{array}$ & $\begin{array}{r}8 \\
14 \\
18 \\
28 \\
8\end{array}$ & $\begin{array}{l}79 \\
86 \\
83 \\
88 \\
87\end{array}$ & $\begin{array}{l}-52 \\
-46 \\
-51 \\
-49 \\
-55\end{array}$ & $\begin{array}{r}1.59 \\
8.31 \\
17.97 \\
1.50 \\
1.60\end{array}$ & $\begin{array}{r}.14 \\
.41 \\
1.18 \\
.10 \\
.14\end{array}$ & $\begin{array}{l}2 A \\
1 \\
1 \\
1 \\
2 A\end{array}$ & $\begin{array}{l}z+014.2 \\
z+016 \\
z+024 \\
z+033 \\
z+051.2\end{array}$ & $\begin{array}{r}+01.73 \\
.09 .73 \\
.03 .57\end{array}$ & $\begin{array}{l}3<456.0 \\
3 C 459.0\end{array}$ & $\begin{array}{l}P \\
P \\
P \\
P\end{array}$ & $\begin{array}{ll}G & N \\
G & N \\
G & \end{array}$ \\
\hline $\begin{array}{l}2344+092 \\
2345+048 \\
2349-014 \\
2350+057\end{array}$ & $\begin{array}{l}\mathbf{s} \\
\mathbf{s} \\
\mathbf{5} \\
\mathbf{s}\end{array}$ & $\begin{array}{l}23 \\
23 \\
23 \\
23\end{array}$ & $\begin{array}{l}44 \\
45 \\
49 \\
50\end{array}$ & $\begin{array}{l}03.90 \\
58.45 \\
22.39 \\
21.11\end{array}$ & $\begin{array}{l}.07 \\
.10 \\
.05 \\
.06\end{array}$ & $\begin{array}{r}09 \\
04 \\
-01 \\
05\end{array}$ & $\begin{array}{l}14 \\
50 \\
25 \\
43\end{array}$ & $\begin{array}{l}05.2 \\
53.6 \\
57.5 \\
51.1\end{array}$ & $\begin{array}{r}.7 \\
1.2 \\
.7 \\
.7\end{array}$ & $\begin{array}{l}8 \\
6 \\
8 \\
5\end{array}$ & $\begin{array}{l}13 \\
11 \\
14 \\
9\end{array}$ & $\begin{array}{l}97 \\
95 \\
92 \\
98\end{array}$ & $\begin{array}{l}-50 \\
-54 \\
-60 \\
-54\end{array}$ & $\begin{array}{l}2.51 \\
1.47 \\
4.11 \\
3.56\end{array}$ & $\begin{array}{l}.16 \\
.12 \\
.26 \\
.22\end{array}$ & $\begin{array}{l}1 \\
2 A R \\
1 R \\
2 A\end{array}$ & $\begin{array}{l}z+073.5 \\
z+077 \\
z-082 \\
z+084\end{array}$ & $\begin{array}{l}+09.74 \\
+04.83 \\
-01.61 \\
+05.86\end{array}$ & & $\begin{array}{l}P \\
P \\
P\end{array}$ & G \\
\hline
\end{tabular}

2, 2A, 2B-lobe-shift probability $\sim 5 \%$; class 3 -lobeshift probability about $20 \%$. An " $R$ " appended to these class numbers means that there is evidence of resolution. "RR" indicates severe resolution. The lobe-shift rate appears to be about $50 \%$ for classes $3 R$ and $3 \mathrm{RR}$.

Column (9): Other names. B2 $=$ Bologna, $\mathbf{P}=$ Parkes, $\mathrm{G}=$ Green Bank, $\mathrm{AO}=$ Arecibo, $\mathrm{N}=$ NRAO.

\section{TEXAS-OHIO POSITION COMPARISON}

The distribution of position differences between the Ohio positions and the BSI positions is shown in Figure 1 for three flux density ranges. This gives an indication only of the Ohio position errors, since the BSI position errors are much smaller. The extended tails of the distributions are caused primarily by the presence of those blend components which were seen by the BSI as separate sources. If one ignores the tails of the distributions, the position differences are consistent with the Ohio position errors as given by Brundage et al. (1971), namely $\sigma_{\alpha} \approx 10^{\text {s }}$ and $\sigma_{\delta} \approx 8^{\prime}$ for $S_{1415}=0.2 \mathrm{Jy}, \sigma_{\alpha} \approx 7^{\mathrm{s}}$ and $\sigma_{\delta} \approx 5^{\prime}$ for $S_{1415}=$ $0.4 \mathrm{Jy}$, and $\sigma_{\alpha} \approx 4^{\mathrm{s}}$ and $\sigma_{\delta} \approx 3^{\prime}$ for $S_{1415}=1.0 \mathrm{Jy}$.

\section{OPTICAL DATA}

Accurate positions were determined on the National Geographic Society-Palomar Observatory Sky Survey (PSS) plates for all objects within 25" of each radio source in the sample, and also for a few (typically three) stars within $4^{\prime}$ of each radio source for use as secondary position standards.

\section{a) Machine Measurements}

Measurements were made with a two-coordinate laser-interferometer measuring machine, built for the 


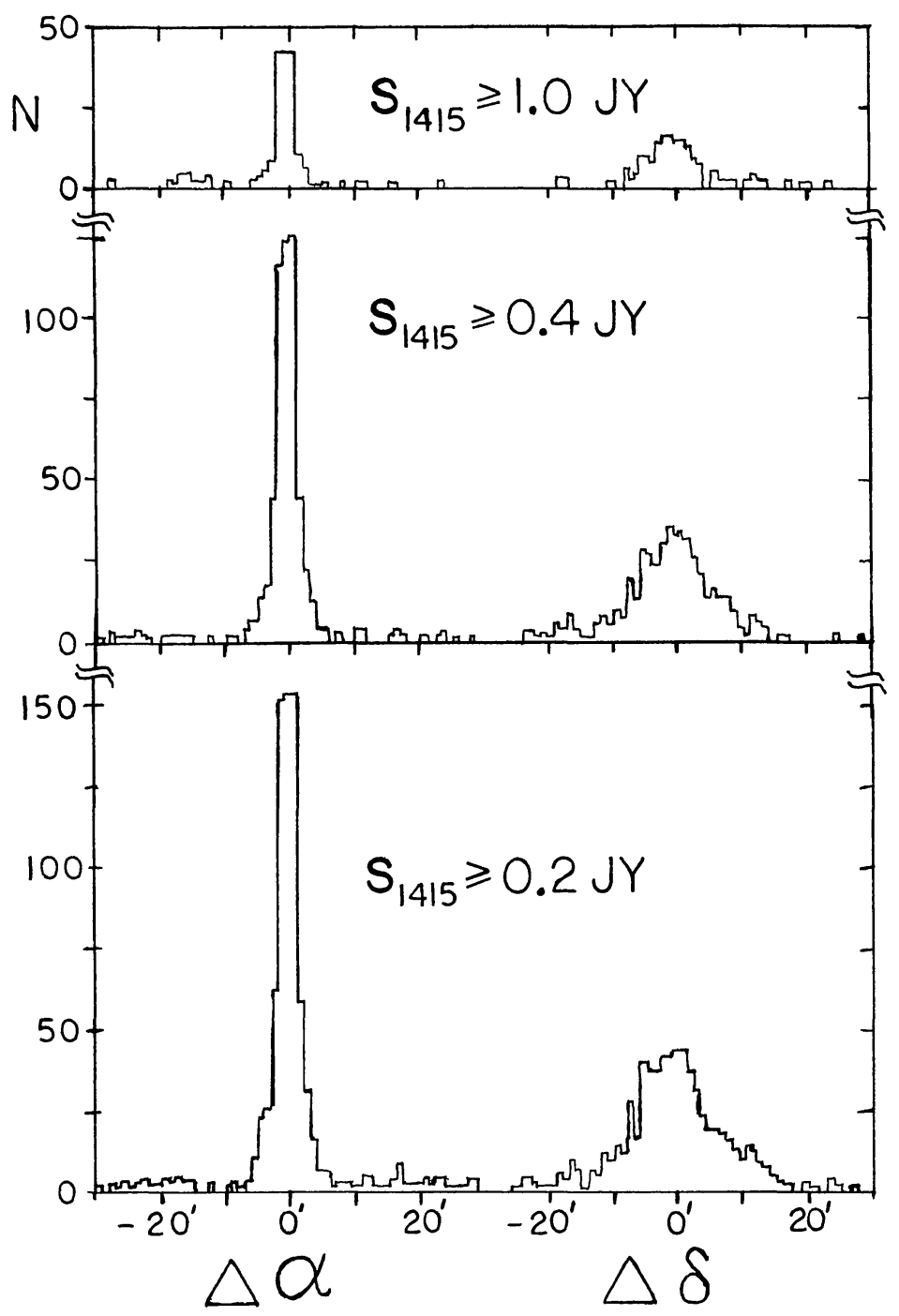

FIG. 1.-Ohio-Texas position differences

University of Texas Radio Astronomy Observatory by Opto Mechanisms, Inc. Position standards were from the Smithsonian Astrophysical Observatory (SAO) catalog. A local second-order least-squares fit was done in a $2^{\circ}$ square centered on each radio source. About 10 to 12 reference stars determined each fit. The equations of condition were

$$
\begin{aligned}
\alpha-\alpha_{0}= & G_{1}+G_{2} x+G_{3} y+G_{4} x^{2}+G_{5} y^{2} \\
& +G_{6} x y, \\
\delta-\delta_{0}= & D_{1}+D_{2} x+D_{3} y+D_{4} x^{2}+D_{5} y^{2} \\
& +D_{6} x y,
\end{aligned}
$$

where $G_{i}$ and $D_{i}(i=1,2, \ldots, 6)$ are parameters to be determined, $(\alpha, \delta)$ are the equatorial coordinates, $\left(\alpha_{0}, \delta_{0}\right)$ denote the field center, and $(x, y)$ are rec- tangular coordinates on the plate relative to the plate center. If an insufficient number of stars were available in the $2^{\circ}$ square area, a linear fit was done, involving only the first three terms in the above expressions.

For each field, measurements were made on both red and blue PSS plates. Positions were determined separately on the two plates. The mean of the two positions is quoted in Table 3 for objects which occurred on both plates.

The internal standard errors of the least-squares fit were in the range 0.2 to 0.4 . Estimates of the measurers' personal equation effects were also in this range. As yet undiagnosed problems with the measuring apparatus appear to contribute an additional uncertainty of \pm 0 ". 3 to \pm 0 ". 4 . Thus the overall standard errors in the resulting optical positions given in Table 3 are 0.5 to 0 ".7. In $\S$ VI we shall see that these estimates are consistent with comparisons with other accurate optical positions. 
TABLE 3

Optical Data for 664 Radio Sources

In POSITION(1950.0)

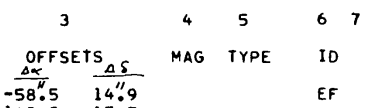

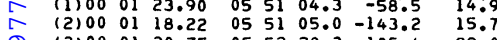
$\operatorname{lil}_{\text {Ifl }}(3) 00 \quad 01 \quad 20.75 \quad 05 \quad 52 \quad 28.3 \quad-105.4 \quad 99.0$

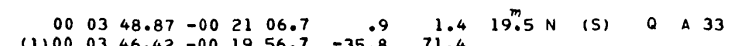

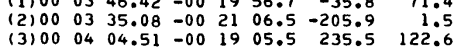

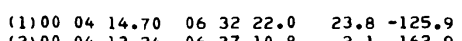

(2) $0004 \quad 04 \begin{array}{llllll}13.24 & 06 & 37 & 10.8 & 2.1 & 162.9\end{array}$

$\begin{array}{llllllllllll}00 & 04 & 33.20 & 05 & 19 & 27.7 & 4.5 & -3.4 & 20.0 & 8 & D & \text { PG B } 38\end{array}$

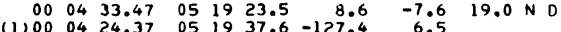

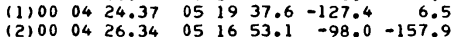

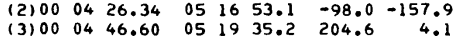

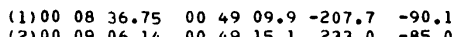

EF

$\begin{array}{lllllllllll}00 & 09 & 01.11 & 08 & 07 & 14.7 & .2 & 1.4 & 20.0 & \text { B } & \text { (S) PO A } 38\end{array}$

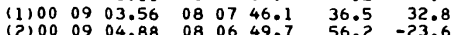

(1)

$\begin{array}{llllllll}00 & 10 & 37 & 00 & 34.8 & 13 \mathrm{SF} & (20.0) & \mathrm{EF}\end{array}$

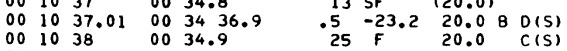

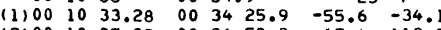

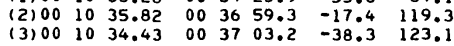

(1) $001126.88 \quad 0527 \quad 48.8 \quad-9.4 \quad-43.5$

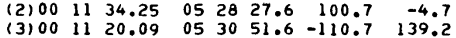

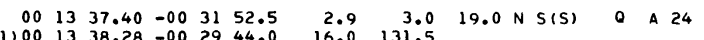

(1) $100 \quad 13 \quad 38.28-002944.0 \quad 16.0 \quad 131.5$

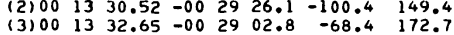

$\begin{array}{llllllllllll}00 & 14 & 39.83 & 08 & 09 & 28.6 & -12.8 & -4.1 & 17.5 & R & S(S) & E F\end{array}$

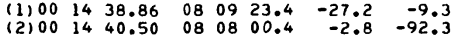

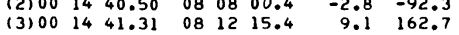

(1) $00 \quad 16 \quad 23.68 \quad 0922 \quad 52.7 \quad 45.8 \quad-35.2$

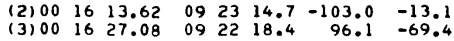

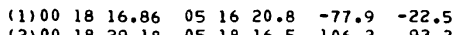

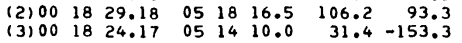

(1) $00 \quad 18 \quad 53.58 \quad-01 \quad 1234.6 \quad 27.7 \quad-19.0$

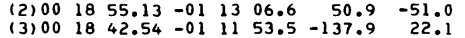

$\begin{array}{llllllllll}00 & 19 & 51.63 & -00 & 01 & 42.5 & .1 & .9 & 20.0 & R\end{array}$ PG A 28

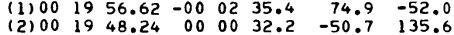

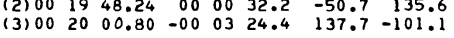

$\begin{array}{llllllllllll}00 & 20 & 46.30 & 04 & 12 & 09.9 & 16.4 & 3.0 & 19.0 & B & \text { (S) } & \text { EF }\end{array}$

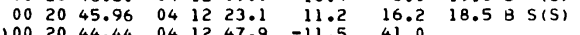

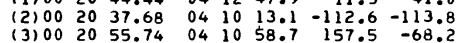

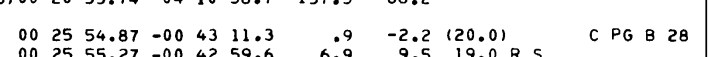

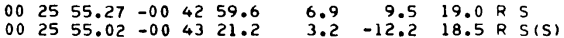

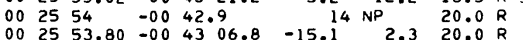

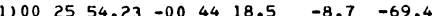

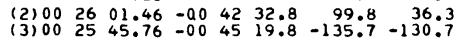

$\begin{array}{llllllllllll}00 & 25 & 59.63 & 00 & 38 & 35.9 & 2.1 & .8 & 17.0 \mathrm{~N} \mathrm{~S}(\mathrm{~S}) & G & A & 41\end{array}$

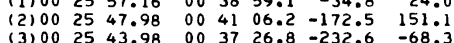

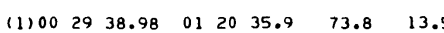

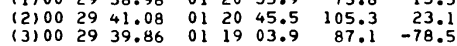

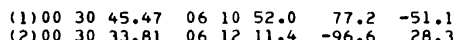

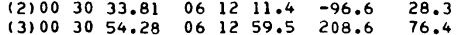

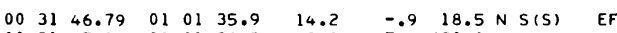

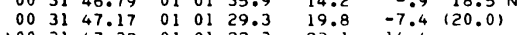

(2) $00 \begin{array}{lllllll}31 & 38.26 & 01 & 00 & 45.9 & -113.8 & -50.9\end{array}$

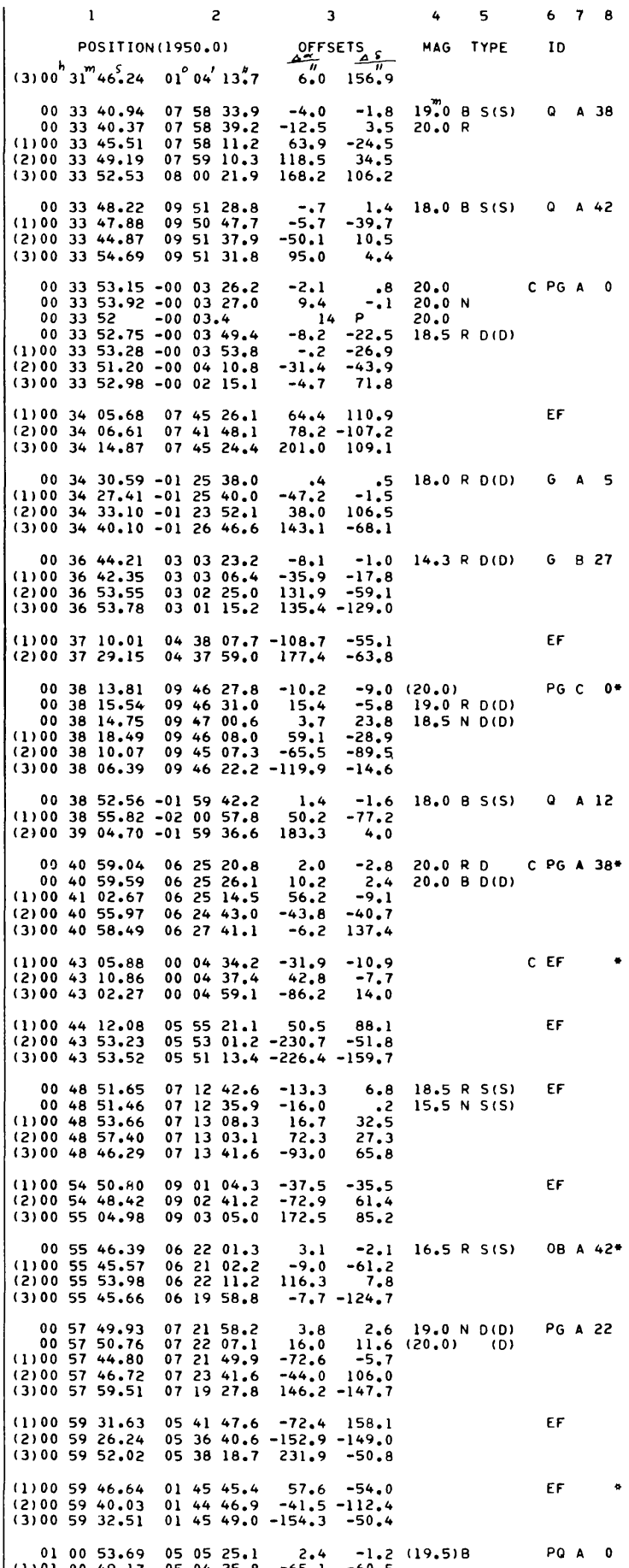

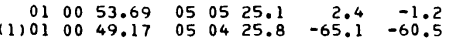

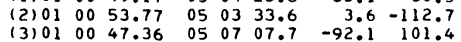


TABLE 3-Continued

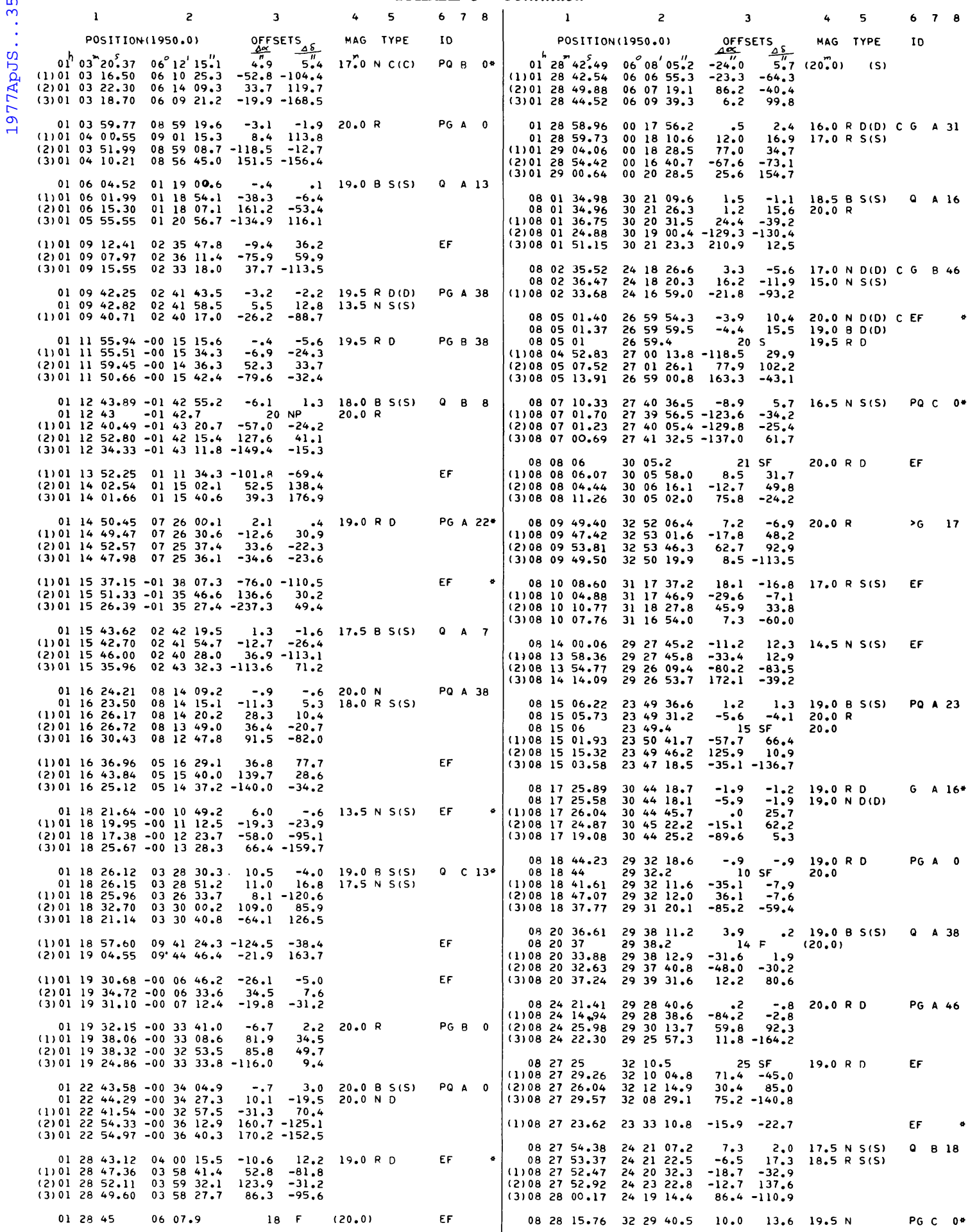

$012845 \quad 06 \quad 07.9$

$18 \mathrm{~F} \quad(20.0)$

$\begin{array}{llllll}08 & 28 & 15.76 & 32 & 29 & 40.5\end{array}$ 
TABLE 3-Continued

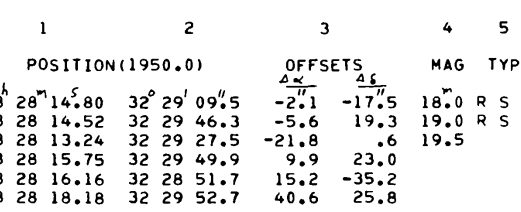

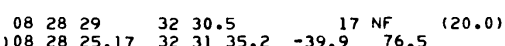

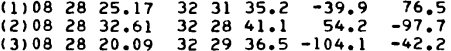

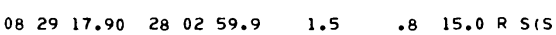

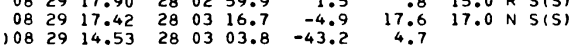

(2) $0829 \begin{array}{llllll}13.96 & 28 & 02 & 56.0 & -50.7 & -3.1\end{array}$

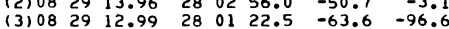

$\begin{array}{lllllllll}08 & 31 & 39.86 & 24 & 08 & 47.0 & -7.3 & -5.9 & 20.0\end{array}$

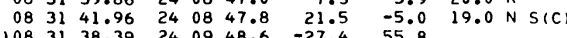

$\begin{array}{llllllll}\text { (1) } 08 & 31 & 38.39 & 24 & 09 & 48.6 & -27.4 & 55.8\end{array}$

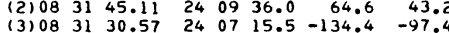

$\begin{array}{lllllllll}08 & 32 & 11.54 & 26 & 44 & 56.7 & 8.1 & -1.4 & 20.0\end{array}$

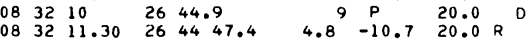

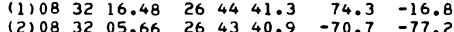

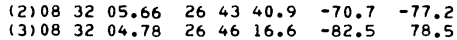

$\begin{array}{lllllllllllll}08 & 32 & 30.63 & 26 & 26 & 12.5 & -15.2 & -19.6 & 20.0 & R & 0\end{array}$

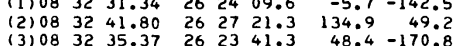

$\begin{array}{lllllllllll}08 & 34 & 42.35 & 25 & 04 & 54.2 & -6.1 & -4.4 & 18.0 & \text { B } & S(S)\end{array}$

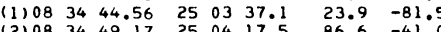

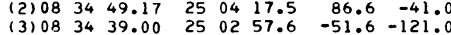

$\begin{array}{lllllllll}08 & 35 & 52.80 & 25 & 37 & 49.9 & -.9 & -.6 & 20.0\end{array}$

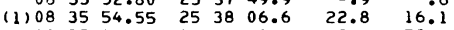

$\begin{array}{rlllllrr}(2) 08 & 35 & 57.09 & 25 & 36 & 58.1 & 57.1 & -52.4 \\ 13) 08 & 35 & 48.13 & 25 & 36 & 20.7 & -64.1 & -89.8\end{array}$

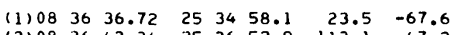

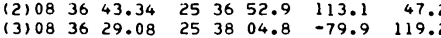

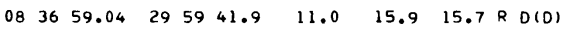

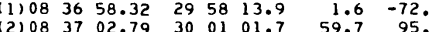

$\begin{array}{lllllllllll}08 & 38 & 06.74 & 32 & 35 & 43.0 & -1.0 & -.7 & 15.0 & R & D(0)\end{array}$

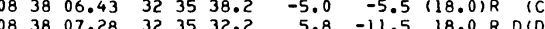

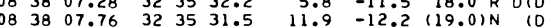

$\begin{array}{lllllllllll}08 & 38 & 06.92 & 32 & 36 & 06.7 & 1.2 & 23.0 & 19.0 & R & 0\end{array}$

$\begin{array}{llllll}1108 & 38 & 01.05 & 32 & 36 & 36.0\end{array}$

(2) $08 \quad 3800.68 \quad 32 \quad 33 \quad 56.0 \quad-77.7-107.7$

$\begin{array}{lllll}32 & 34 & 27.4 & -210.1 & -76.3\end{array}$

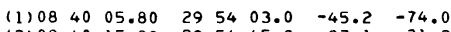

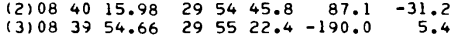

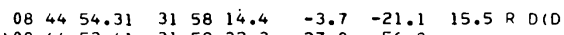

(1) $0844 \quad 52.41 \quad 31 \quad 59 \quad 32.3 \quad-27.8 \quad 56.8$

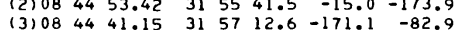

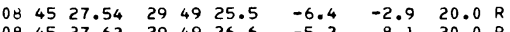

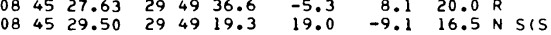

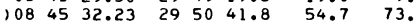

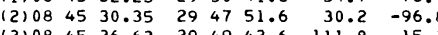

(3) $08 \quad 45 \quad 36.63 \quad 29 \quad 4943.6 \quad 111.8 \quad 15.1$

$\begin{array}{llllllllllll}08 & 46 & 37.26 & 31 & 37 & 22.4 & 15.7 & -5.6 & 20.0 & 8 & \text { (D) } & E F\end{array}$

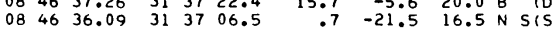

$\begin{array}{llllllrr}(1) 08 & 46 & 39.96 & 31 & 37 & 16.0 & 50.2 & -12.1 \\ (2) 08 & 46 & 24.91 & 31 & 35 & 11.4 & -142.1 & -136.7\end{array}$

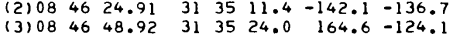

$\begin{array}{lllllllll}11 & 08 & 51 & 23.35 & 30 & 20 & 05.3 & -100.4 & -82.6\end{array}$

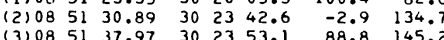

$\begin{array}{llllllllllllll}08 & 53 & 00.59 & 29 & 10 & 06.3 & 1.6 & -.3 & 19.0 & B & C(S) & 0 & A & 15\end{array}$

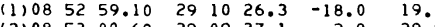

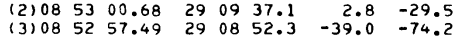

$\begin{array}{lllllllllll}08 & 55 & 11.37 & 28 & 02 & 14.6 & 7.2 & -14.5 & 20.0 & R\end{array}$

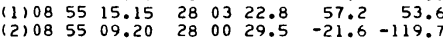

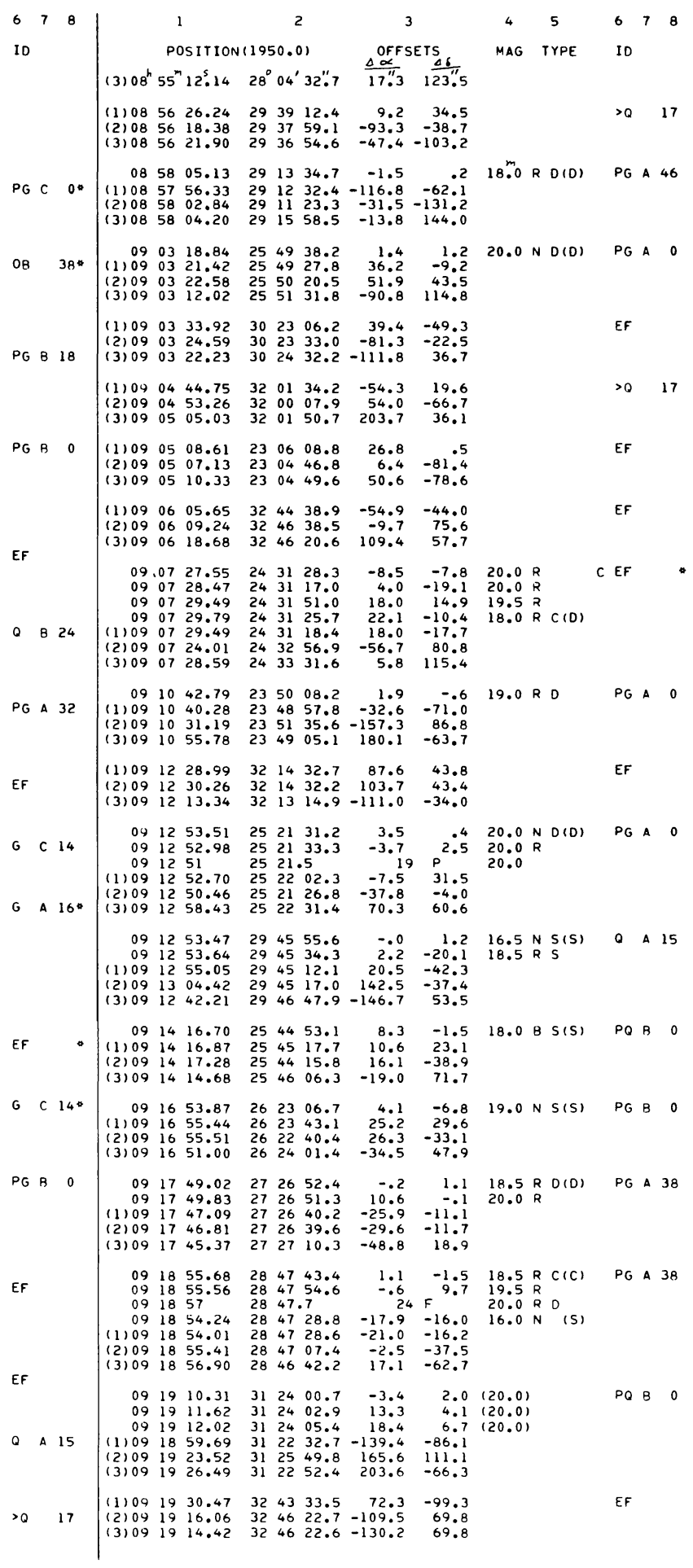


TABLE 3-Continued

\begin{tabular}{|c|c|c|c|c|c|c|c|c|}
\hline 1 & 2 & 3 & 3 & 4 & 5 & 6 & 7 & \\
\hline POSITION & $(1950.0)$ & OFFS & SETS & MAG & TYPE & 10 & & \\
\hline $\begin{array}{ll}20 & 00^{\mathrm{m}} .00 \\
20 & 07.10 \\
20 & 01.86 \\
20 & 06.33 \\
20 & 16.84\end{array}$ & $\begin{array}{lll}31^{\circ} & 12^{\prime} & 18^{\prime \prime} .4 \\
31 & 12 & 29.7 \\
31 & 13 & 31.2 \\
31 & 10 & 31.8 \\
31 & 11 & 54.2\end{array}$ & $\begin{array}{r}2.6 \\
-8.9 \\
-76.2 \\
-18.9 \\
116.1\end{array}$ & $\begin{array}{r}-10.6 \\
10.6 \\
72.1 \\
-107.2 \\
-24.8\end{array}$ & $\begin{array}{l}19.0 \\
19.5\end{array}$ & $\begin{array}{l}B \\
B\end{array}$ & $P Q$ & A 16 & \\
\hline $\begin{array}{ll}20 & 48.46 \\
20 & 53.56\end{array}$ & $\begin{array}{lll}31 & 20 & 48.7 \\
31 & 20 & 16.3\end{array}$ & $\begin{array}{r}-.1 \\
65.3\end{array}$ & $\begin{array}{r}3.5 \\
-29.0\end{array}$ & 18.0 & B $S(S)$ & 0 & A 41 & \\
\hline
\end{tabular}

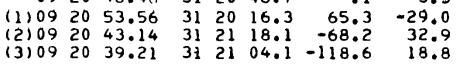

$\begin{array}{llllllllll}09 & 22 & 29.80 & 31 & 00 & 19.8 & -11.9 & -5.7 & (19.0) & \text { EF }\end{array}$

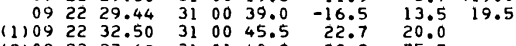

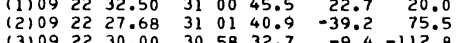

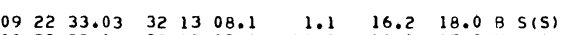

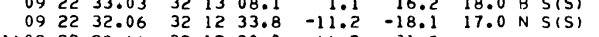

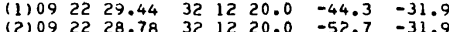

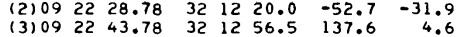

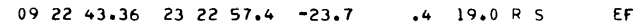

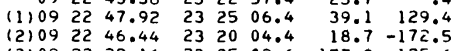

(3) $0922 \quad 32.16 \quad 23 \quad 25 \quad 02.6 \quad-177.9 \quad 125.6$

$\begin{array}{lllllllr}\text { (1) } 09 & 23 & 14.51 & 26 & 12 & 03.2 & 108.2 & -48.4 \\ \text { (2) } 09 & 23 & 16.84 & 26 & 14 & 28.0 & 139.6 & 96.4\end{array}$

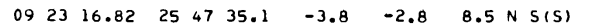

$\begin{array}{rrrrrrrr}\text { (1) } 09 & 23 & 16.82 & 25 & 47 & 35.1 & -3.8 & -2.8 \\ \text { (2) } 09 & 23 & 05.84 & 25 & 47 & 56.2 & 64.1 & 18.4 \\ \text { (2) } & 25 & 49 & 06.5 & -163.1 & 88.6\end{array}$

$\begin{array}{lllllll}\text { (1) } 0925 & 22.02 & 29 & 16 & 17.6 & 49.7 & -64.2\end{array}$

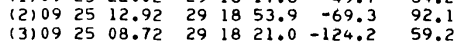

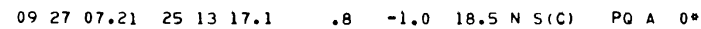

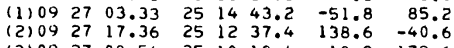

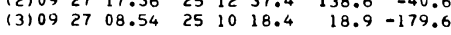

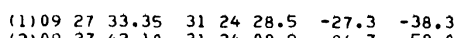

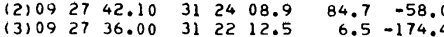

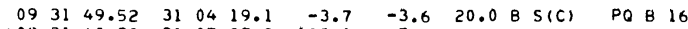

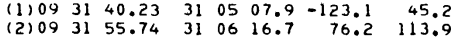

(3) $093201.22 \quad 3104 \quad 40.7 \quad 146.6 \quad 18.0$

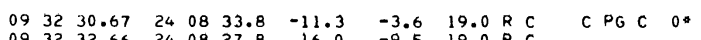

$22.8 \quad 15.0 R \quad S(S)$

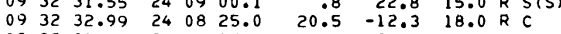

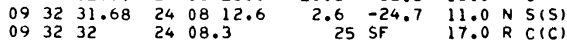

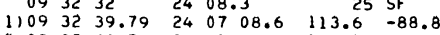

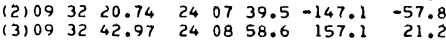

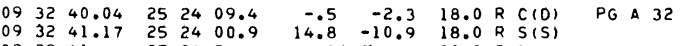

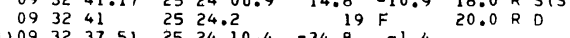

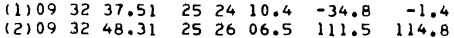

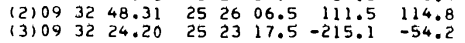

$\begin{array}{llllllll}0432 & 55 & 24 & 10.3 & 7 & 5 P & 20.0 & \text { PQ }\end{array}$

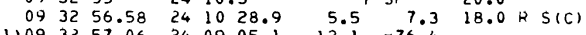

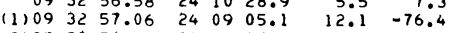

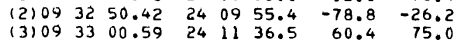

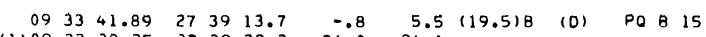

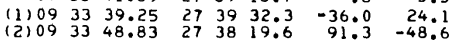

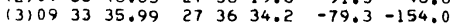

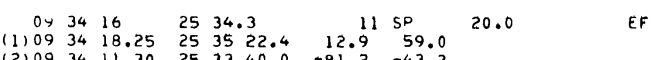

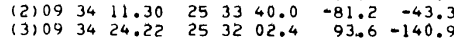

$\begin{array}{llllllllll}04 & 36 & 01.88 & 26 & 12 & 45.7 & -10.3 & -18.8 & 16.0 \mathrm{~N} \mathrm{~S}(\mathrm{~S})\end{array}$

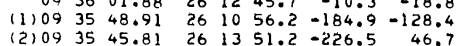

$\begin{array}{llllllllll}09 & 36 & 42.16 & 24 & 42 & 28.4 & 2.1 & 2.5 & 20.0 & R\end{array}$

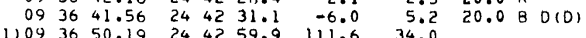

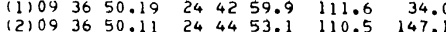

(3) $0936 \quad 30.90 \quad 24 \quad 44 \quad 39.4-151.2 \quad 133.5$

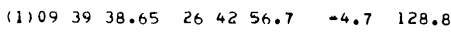

\section{1}

POSITION $(1950.01$ $\begin{array}{llllll}3 & 4 & 5 & 6 & 7 & 8\end{array}$

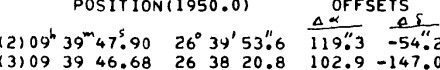

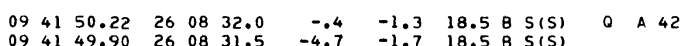

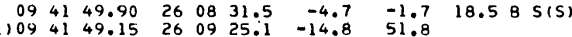

(2) $0941 \quad 42.22 \quad 26 \quad 0921.3 \quad-108.1 \quad 48.0$

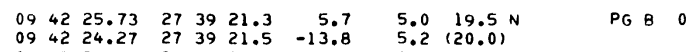

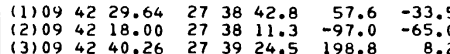

(1) $0942 \quad 43.88 \quad 26 \quad 54 \quad 05.9 \quad 74.0 \quad-38.5$

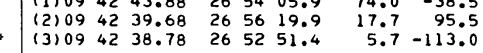

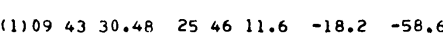

(2) $0943 \quad 19.33 \quad 2549 \quad 06.6-168.8 \quad 116$.

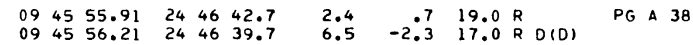

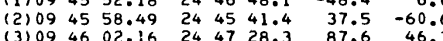

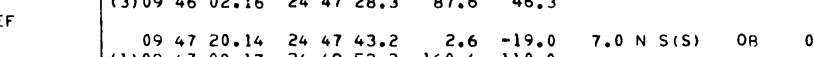

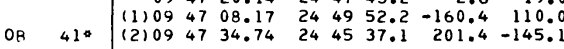

$\begin{array}{lllllllllllll}09 & 49 & 10.01 & 24 & 36 & 36.0 & -2.9 & -2.2 & 18.5 \text { R S } & \text { PG A 7* } \\ 09 & 49 & 09.48 & 24 & 36 & 29.8 & -10.1 & -8.4 & 19.5\end{array}$

$\begin{array}{lllllll}\text { (1) } 0949 & 05.54 & 24 & 35 & 53.0 & -63.8 & -45.2\end{array}$

(2) $094902.87 \quad 243758.2 \quad-100.3 \quad 80.0$

$\begin{array}{lllllllllll}09 & 49 & 12.70 & 28 & 42 & 37.1 & 2.5 & -3.1 & (20.0) & \text { (D) PG A 17* }\end{array}$ (1) $0949 \quad 02.17 \quad 28 \quad 44 \quad 41.5 \quad-136.2 \quad 121.4$

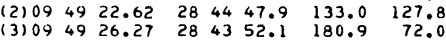

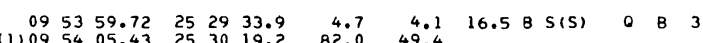

(2) $095352.9925 \quad 3122.4 \quad-86.5 \quad 112.6$

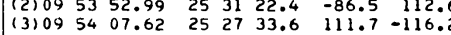

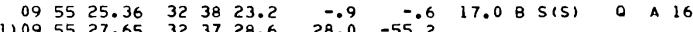

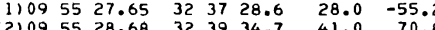

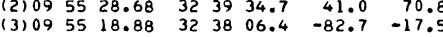

$\begin{array}{lllllll}\text { (1) } 0958 & 33.33 & 25 & 41 & 22.9 & -33.6 & 25.4\end{array}$

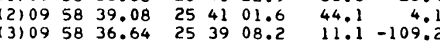

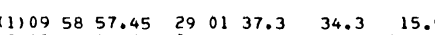

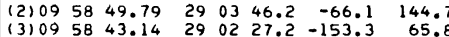

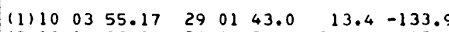

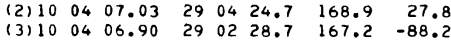

$\begin{array}{lllllllllllll}10 & 04 & 30.82 & 23 & 06 & 07.7 & .9 & -2.7 & 20.0 & B & \text { (s) PQ } A & 0\end{array}$

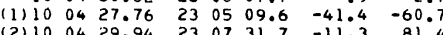

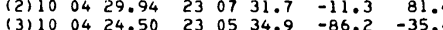

$\begin{array}{llllllllll}10 & 05 & 52.06 & 31 & 16 & 50.0 & -11.5 & 3.7 & (20.0) & E F\end{array}$

(2) $10 \begin{array}{lllllll}0 & 01.78 & 31 & 15 & 57.2 & 113.2 & -49.1\end{array}$

$\begin{array}{llllllll}\text { (2) } 10 & 05 & 43.44 & 31 & 17 & 53.0 & -121.9 & 66.7 \\ \text { (3) } 10 & 05 & 41.18 & 31 & 17 & 34.7 & -150.9 & 48.5\end{array}$

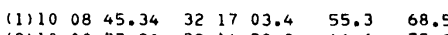

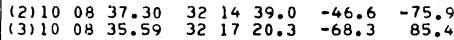

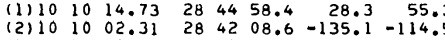

(3) $10 \quad 10 \quad 00.50 \quad 28 \quad 42 \quad 35.3 \quad-158.8 \quad-87.7$

(1) $10 \begin{array}{rrrrrrrrrrr}10 & 10 & 14.68 & 25 & 03 & 28.0 & 16.8 & -1.0 & 20.0 & R & 0\end{array}$

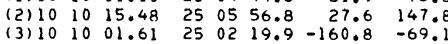

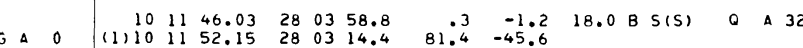

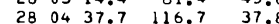

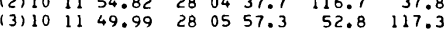

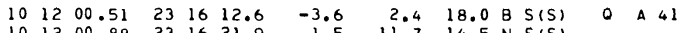

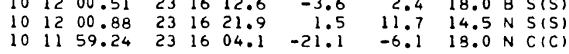


TABLE 3-Continued

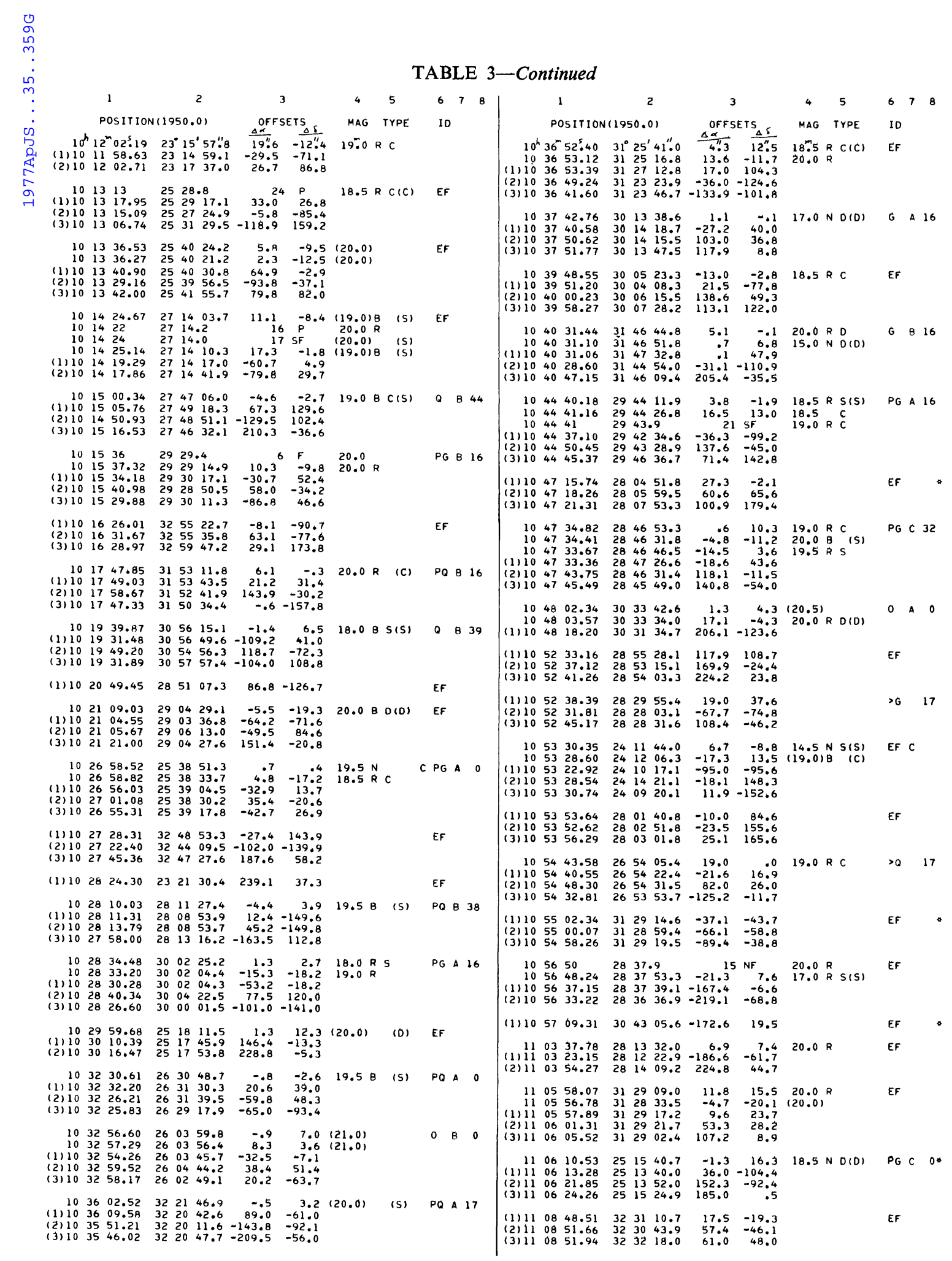


TABLE 3-Continued

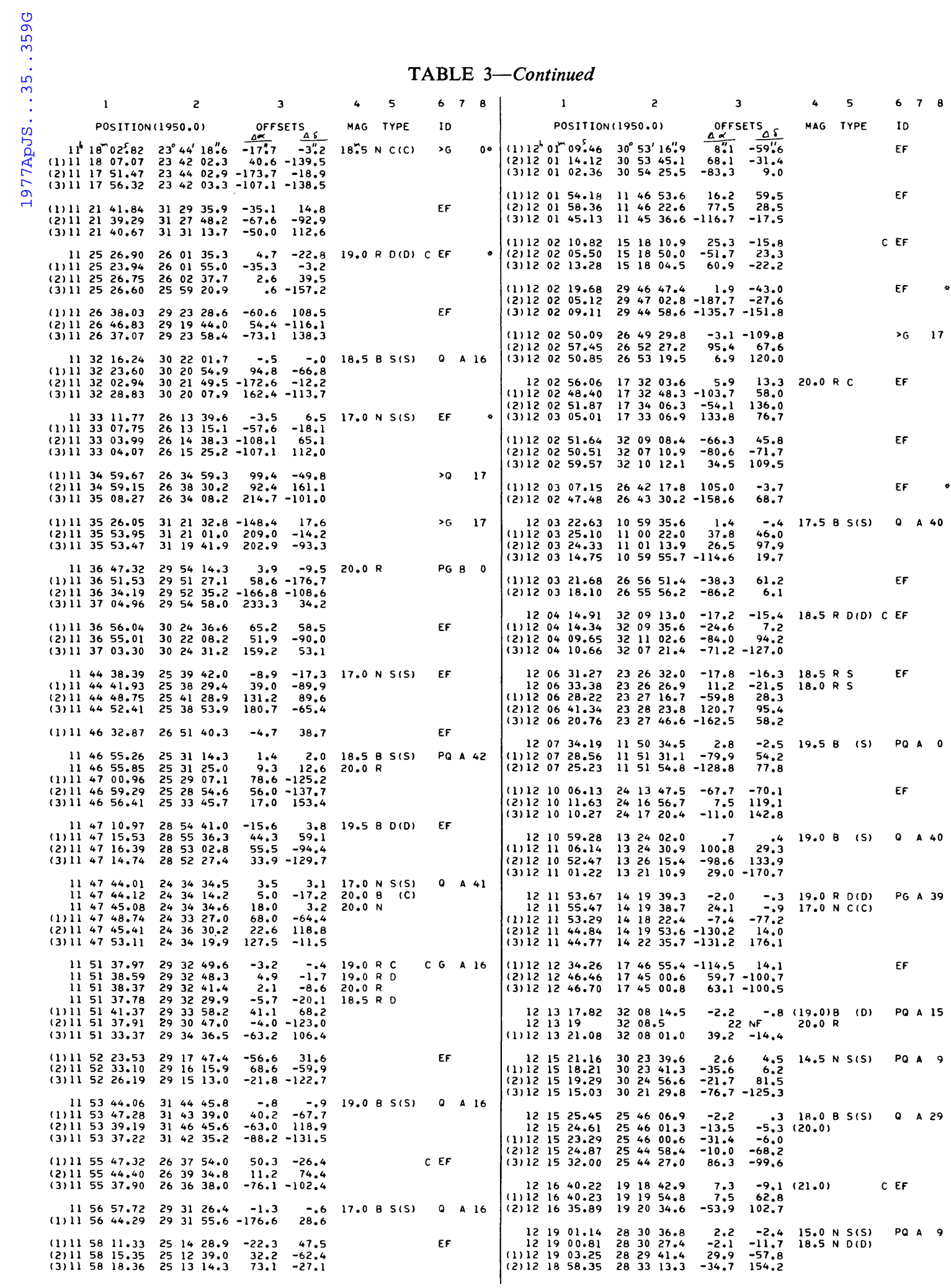


TABLE 3-Continued

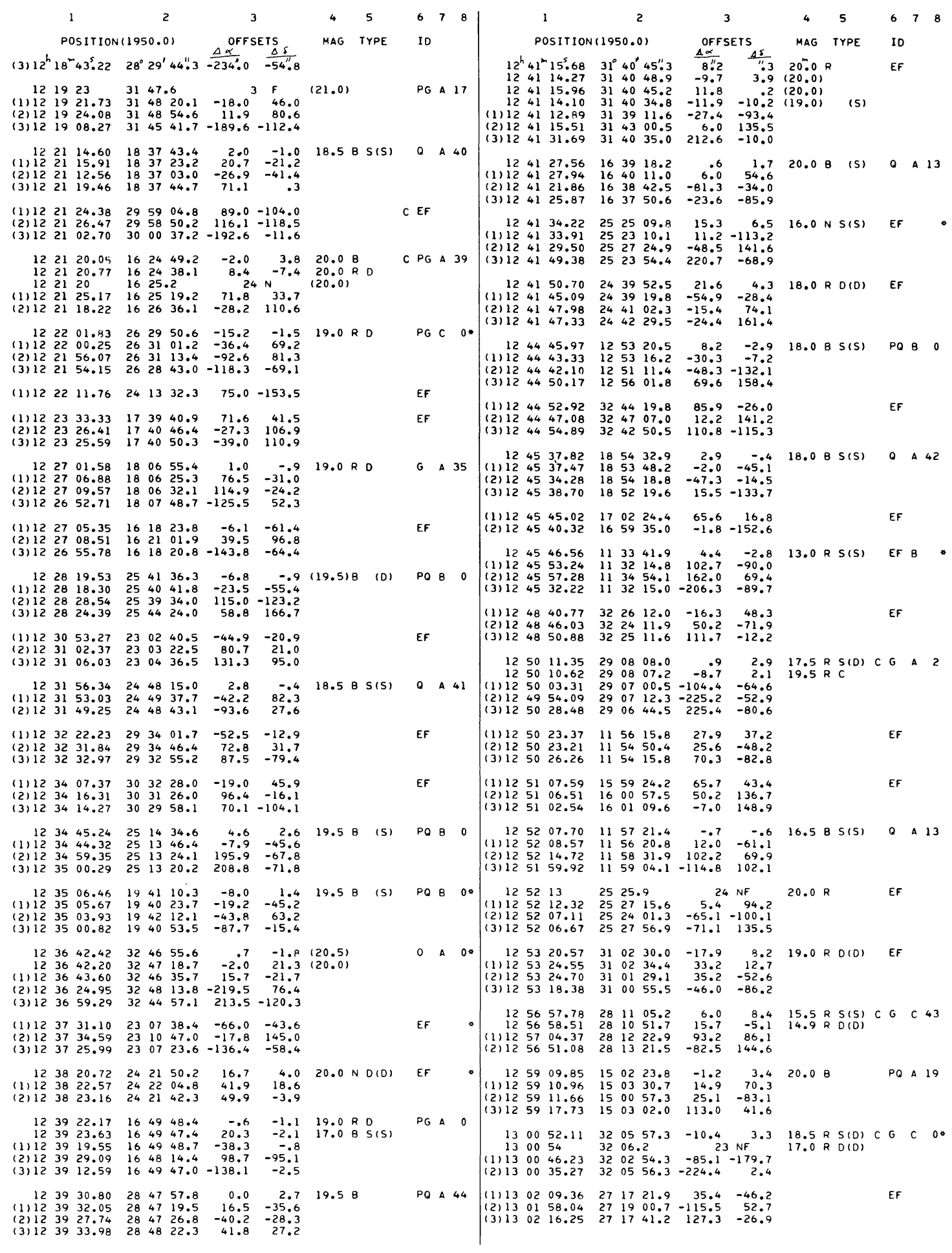




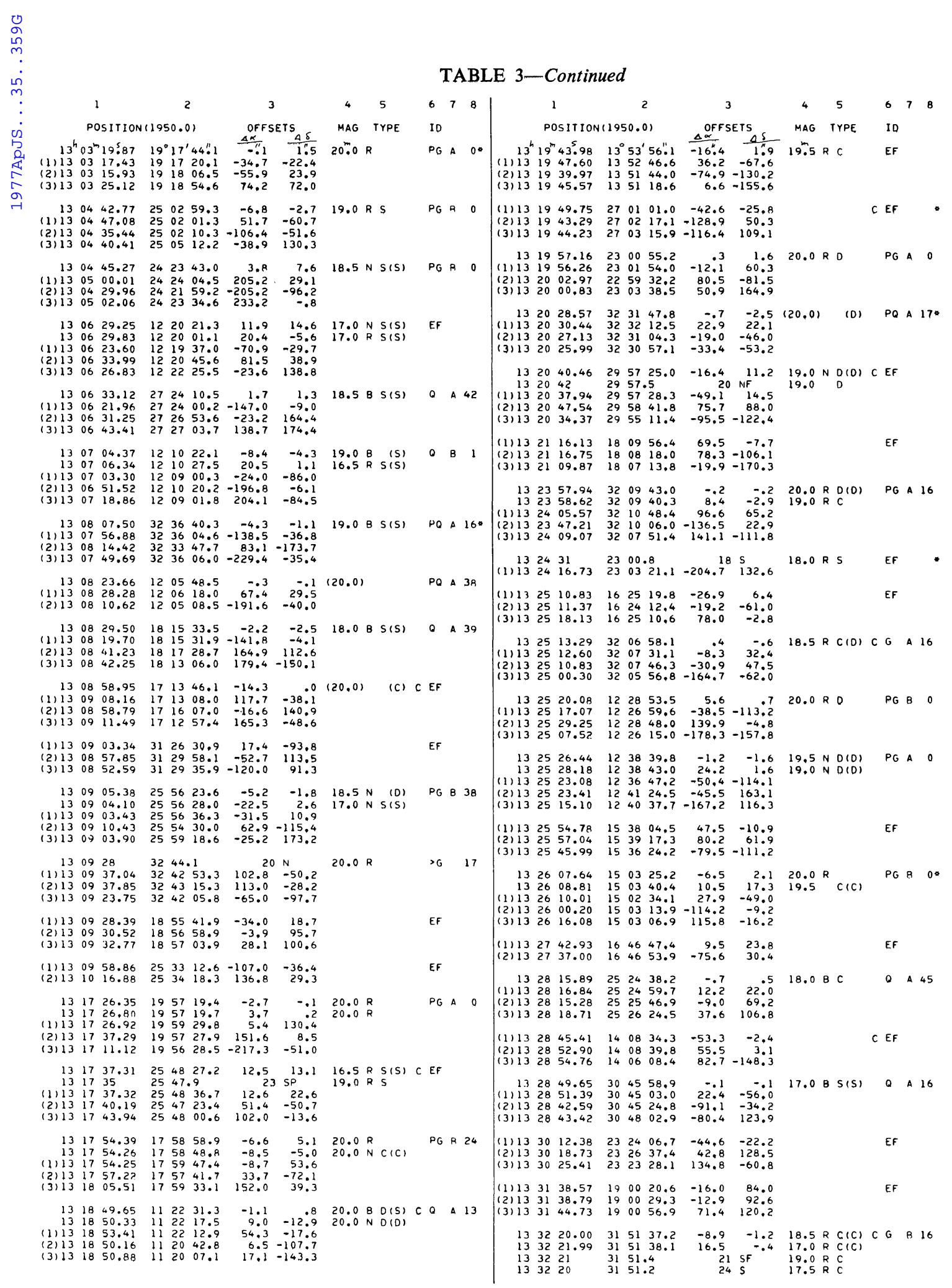


TABLE 3-Continued

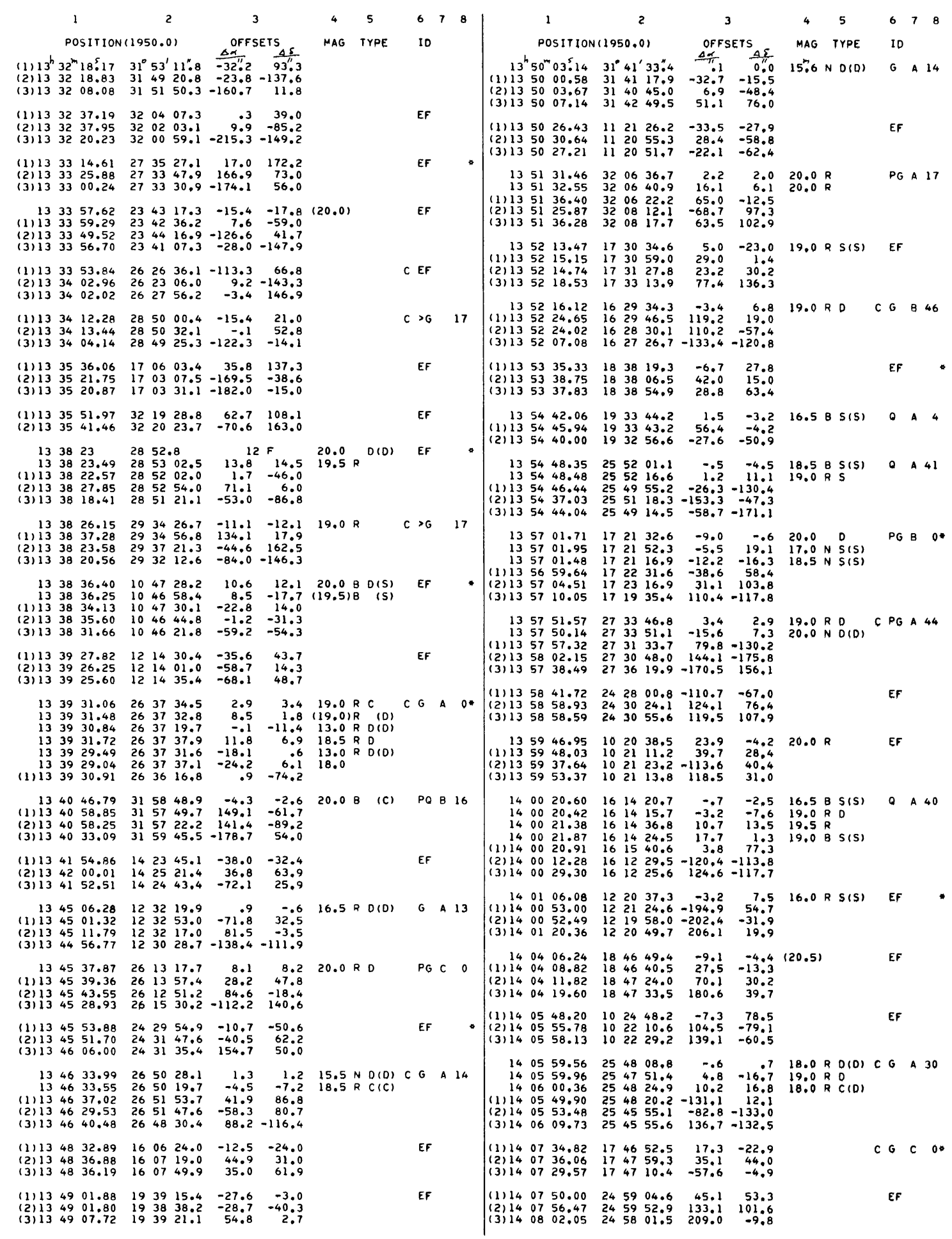


TABLE 3-Continued

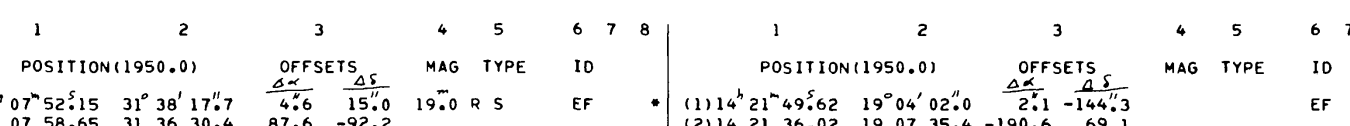

$14^{h} 07^{m} 52^{5} .15 \quad 31^{0} 38^{\prime} 17^{\prime \prime .7} \quad \frac{\Delta \alpha}{4^{* .6}} \quad \frac{\Delta S}{15^{\prime \prime .0}} \quad 19^{m .0}$ R S

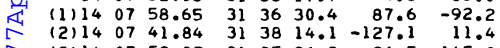

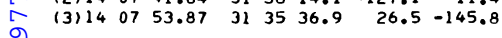
III (1) $14 \quad 07 \quad 54.28 \quad 174747.2 \quad 11.7 \quad-22.5$ $\begin{array}{llllllll}\text { (2) } 14 & 07 & 50.04 & 17 & 47 & 24.3 & -48.8 & -45.5 \\ \text { (3) } 14 & 07 & 47.10 & 17 & 47 & 46.4 & -90.7 & -23.3\end{array}$

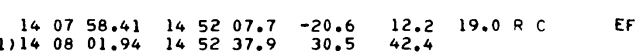

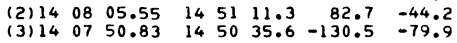

$\begin{array}{lllllllllllllll}14 & 08 & 34 & 14 & 10.9 & & 9 & F & 20.0 & R & D & P G & B & 0\end{array}$

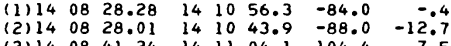

(3) $14 \quad 08 \quad 41.24 \quad 14 \quad 1104.1 \quad 104.4 \quad 7.5$

$\begin{array}{lllllllllll}14 & 09 & 12.62 & 13 & 18 & 10.3 & -12.1 & -15.3 & 20.0 & 0 & 0\end{array}$

(2) 140911.97 13 $16 \quad 59.3 \quad-21.6 \quad-25.0$

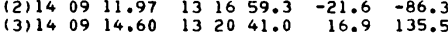

(1) $140951.02 \quad 2923 \quad 39.4 \quad 26.0-131.2$

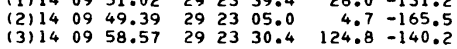

$\begin{array}{llllllll}\text { (1) } 14 & 10 & 06.16 & 11 & 52 & 51.6 & 106.9 & -8.6\end{array}$

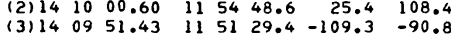

$\begin{array}{lllllllllll}14 & 10 & 13.56 & 29 & 35 & 28.1 & 9.4 & -6.4 & 20.0 & R\end{array} \quad$ EF

(1) 14 10 02.28 29 35 55.2 $137.9 \quad-19.3 \quad 20.0 \mathrm{R}$

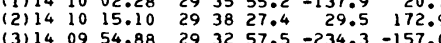

$\begin{array}{lllllllll}\text { (1) } 14 & 10 & 17.18 & 15 & 28 & 45.7 & -36.9 & -27.0 & \text { C EF }\end{array}$

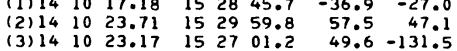

$\begin{array}{lllllllll}14 & 13 & 38 & 25 & 23.9 & 17 & \mathrm{NF} & 20.0 & \\ 14 & 13 & 38 & 25 & 23.4 & 24 & \mathrm{SF} & 20.0 & \text { (D) }\end{array}$

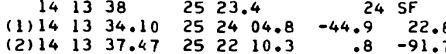

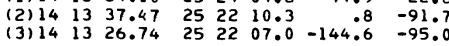

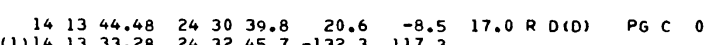

(1) $14 \begin{array}{lllllll}13 & 33.28 & 24 & 32 & 45.7 & -132.3 & 117.3\end{array}$

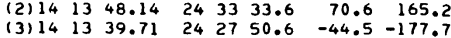

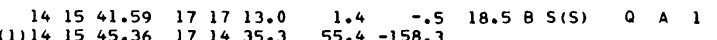

$\begin{array}{llllllll}\text { (1) } 14 \quad 17 & 15.56 & 30 & 20 & 50.2 & 26.7 & -92.3 & \text { EF }\end{array}$

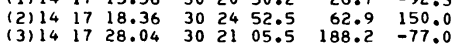

$\begin{array}{llllllllll}14 & 17 & 18.83 & 14 & 27 & 10.3 & 6.3 & 16.0 & 12.0 \mathrm{~N} \mathrm{~S}(S) \text { EF }\end{array}$

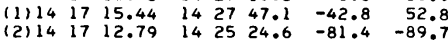

$\begin{array}{rrrrrrrr}\text { (1) } 14 & 17 & 48.93 & 27 & 15 & 33.4 & 56.7 & 6.8 \\ \text { (2) } 14 & 17 & 34.15 & 27 & 16 & 07.7 & -140.3 & 41.1\end{array}$

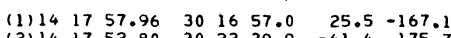

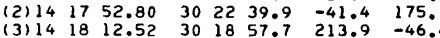

$\begin{array}{llllllllll}14 & 19 & 19.31 & 31 & 32 & 43.1 & .4 & 2.3 & 19.5 & \text { B C }\end{array}$ PO A 16

(1) $144 \begin{array}{llllllll}19 & 20.65 & 31 & 32 & 40.7 & 17.6 & -. .1 \\ 114.16 & 31 & 31 & 01.4 & 62.4 & -99.3\end{array}$

(2) $14 \quad 1923.16 \quad 3134 \quad 46.0 \quad 49.6 \quad 125.3$

$\begin{array}{llllllllll}\text { (1) } 14 & 19 & 33.40 & 18 & 02 & 21.4 & 26.5 & -12.0 & \\ \text { (2) } 14 & 19 & 34.50 & 18 & 02 & 32.0 & 42.1 & -1.3 & \text { EF }\end{array}$

(3) $14 \quad 1933.52 \quad 1801 \quad 59.9 \quad 28.2 \quad-33.5$

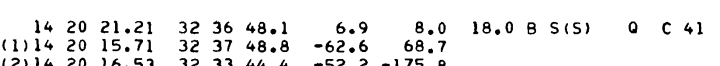

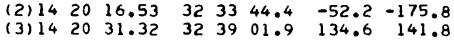

$142040.03 \quad 194913.2 \quad-14.2 \quad 18.4 \quad 19.5 R$ D $\quad G \quad C 6^{\circ}$

(1) $14 \quad 20 \quad 37.98 \quad 194911.1 \quad-43.0 \quad 16.39$

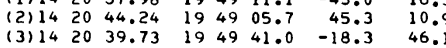

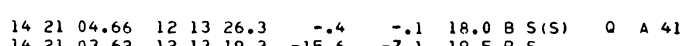

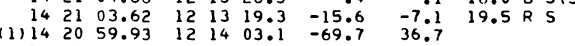

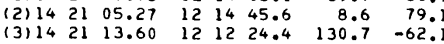

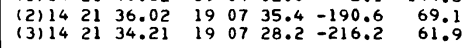

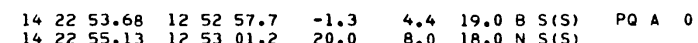

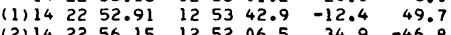

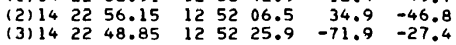

$\begin{array}{llllllllll}11114 & 24 & 45.27 & 10 & 37 & 00.2 & -87.1 & -3.6 & E F\end{array}$

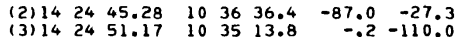

(1) $14 \quad 25 \quad 22.95 \quad 26 \quad 44 \quad 34.2 \quad 20.4 \quad-17.4$

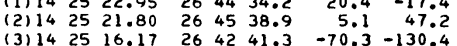

$\begin{array}{rrrrrrrrr}14 & 25 & 28.32 & 28 & 46 & 44.4 & -2.6 & -5.5 & 16.5 \mathrm{~N} S(S)\end{array}$

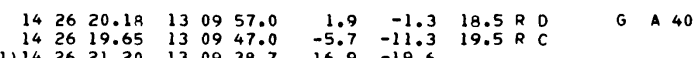

$\begin{array}{llllllrr}\text { (1) } 14 & 26 & 21.20 & 13 & 09 & 38.7 & 16.9 & -19.6 \\ \text { (2) } 14 & 26 & 27.03 & 13 & 08 & 55.5 & 101.9 & -62.8\end{array}$

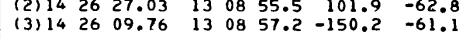

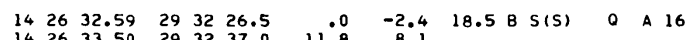

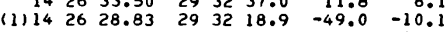

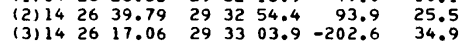

$\begin{array}{lllllllllll}14 & 27 & 28.40 & 30 & 34 & 17.5 & -19.7 & -15.1 & 17.0 \mathrm{~N} S(S) & E F\end{array}$

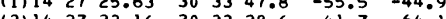

(3) $14 \quad 27 \quad 39.93 \quad 303728.6 \quad 129.1 \quad 175.9$

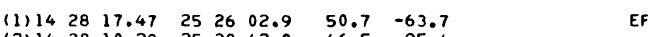

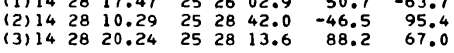

$\begin{array}{llllllllll}14 & 28 & 48.18 & 26 & 03 & 16.6 & -.1 & -3.9 & 20.0 & R\end{array}$ PG A 0

(2) $14 \quad 28 \quad 53.62 \quad 26 \quad 02 \quad 31.4 \quad 73.2 \quad-49.1$

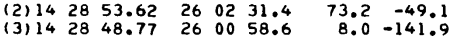

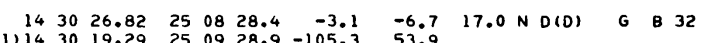

(1) $143019.29 \quad 250928.9-105.3 \quad 53.9$

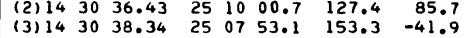

(1) $14 \quad 3036.10 \quad 17 \quad 1557.9-70.8-17.3$

$\begin{array}{llllllll}14 & 30 & 36.10 & 17 & 15 & 57.9 & -70.8 & -17.3\end{array}$

(3) $1430 \quad 44.38 \quad 17 \quad 14 \quad 40.1 \quad 47.9 \quad-95.1$

$\begin{array}{llllllllll}\text { (1) } 14 & 31 & 13.65 & 16 & 20 & 32.4 & 102.1 & -8.9 & \text { EF }\end{array}$

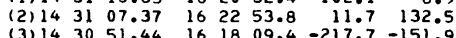

$\begin{array}{rrrrrr}143306 & 3153.8 & 6 & F & 20.0 & P Q 817\end{array}$

(1) $143256.83 \quad 315500.7 \quad-123.3 \quad 75.2$

(2) $1433 \quad 321.20 \quad 3153 \quad 50.7 \quad 187.0 \quad 5.2$

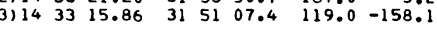

$\begin{array}{llllllll}\text { (1) } 14 & 3320.18 & 23 & 58 & 26.0 & -29.0 & 39.3 & \text { EF }\end{array}$

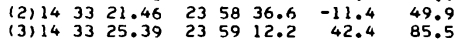

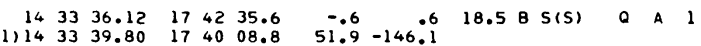

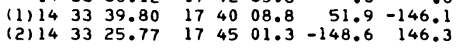

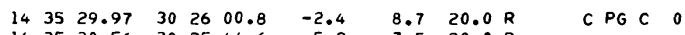

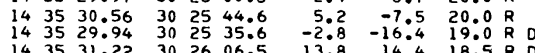

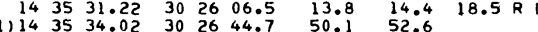

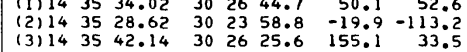

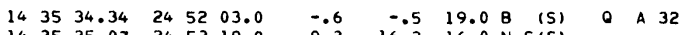

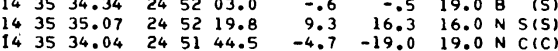

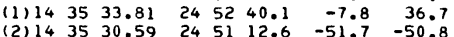

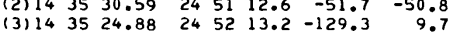

$\begin{array}{llllllll}\text { (1) } 14 & 35 & 45.82 & 28 & 33 & 39.0 & -174.2 & -64.2 \\ \text { (2) } 14 & 36 & 10.03 & 28 & 36 & 58.8 & 144.7 & 135.6\end{array}$

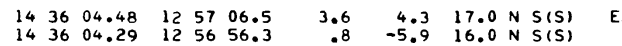

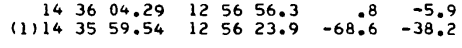


TABLE 3-Continued

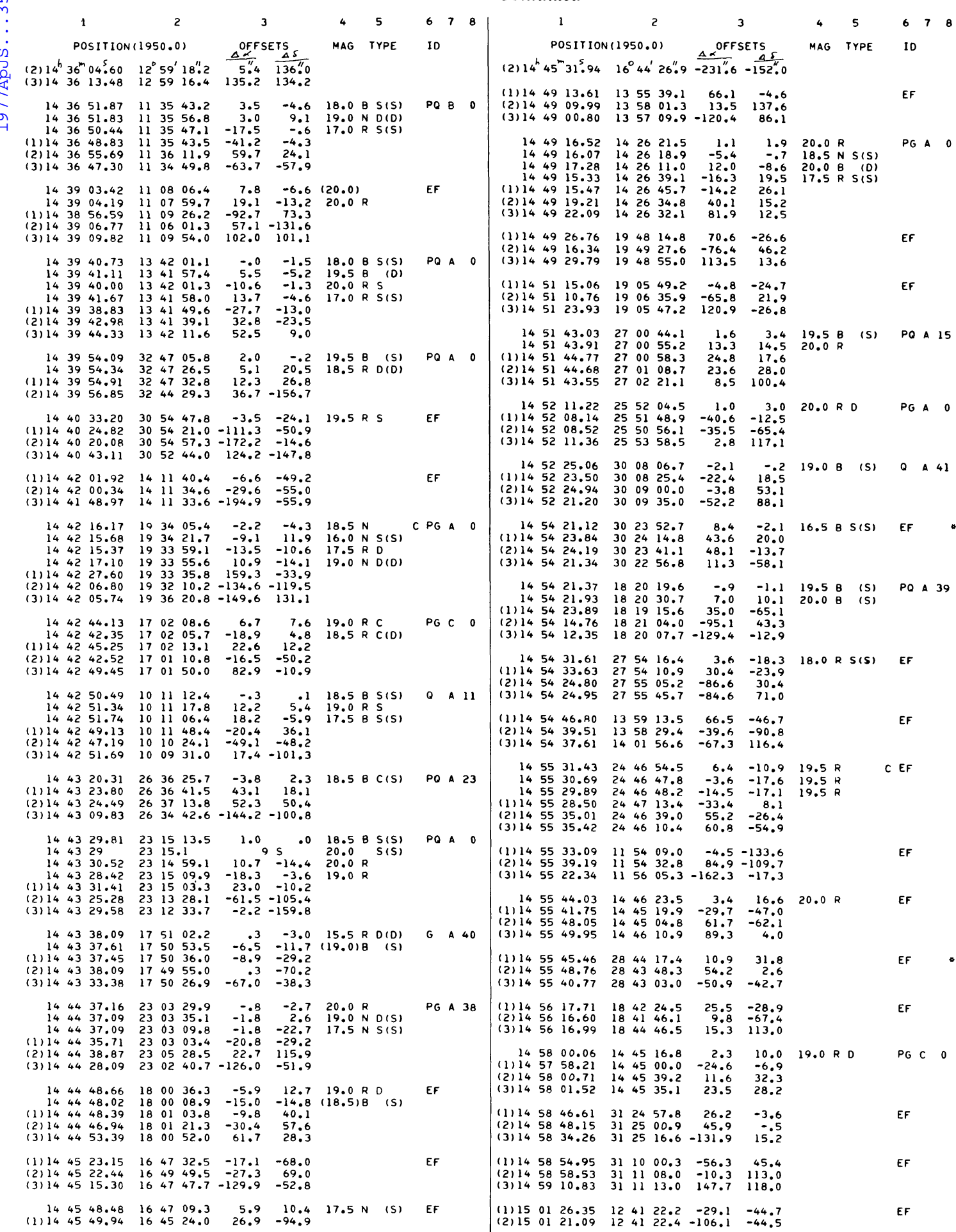


TABLE 3-Continued

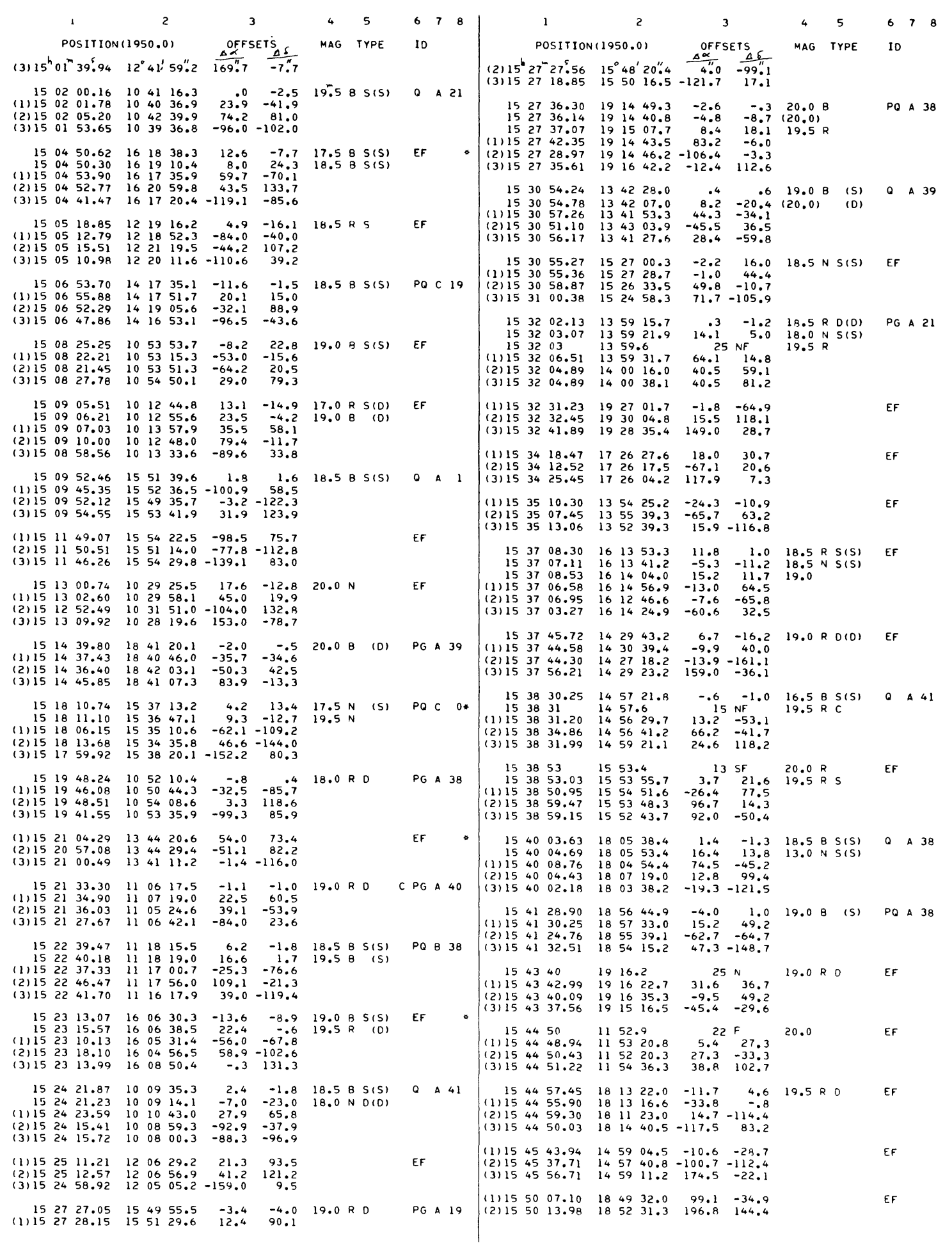


TABLE 3-Continued

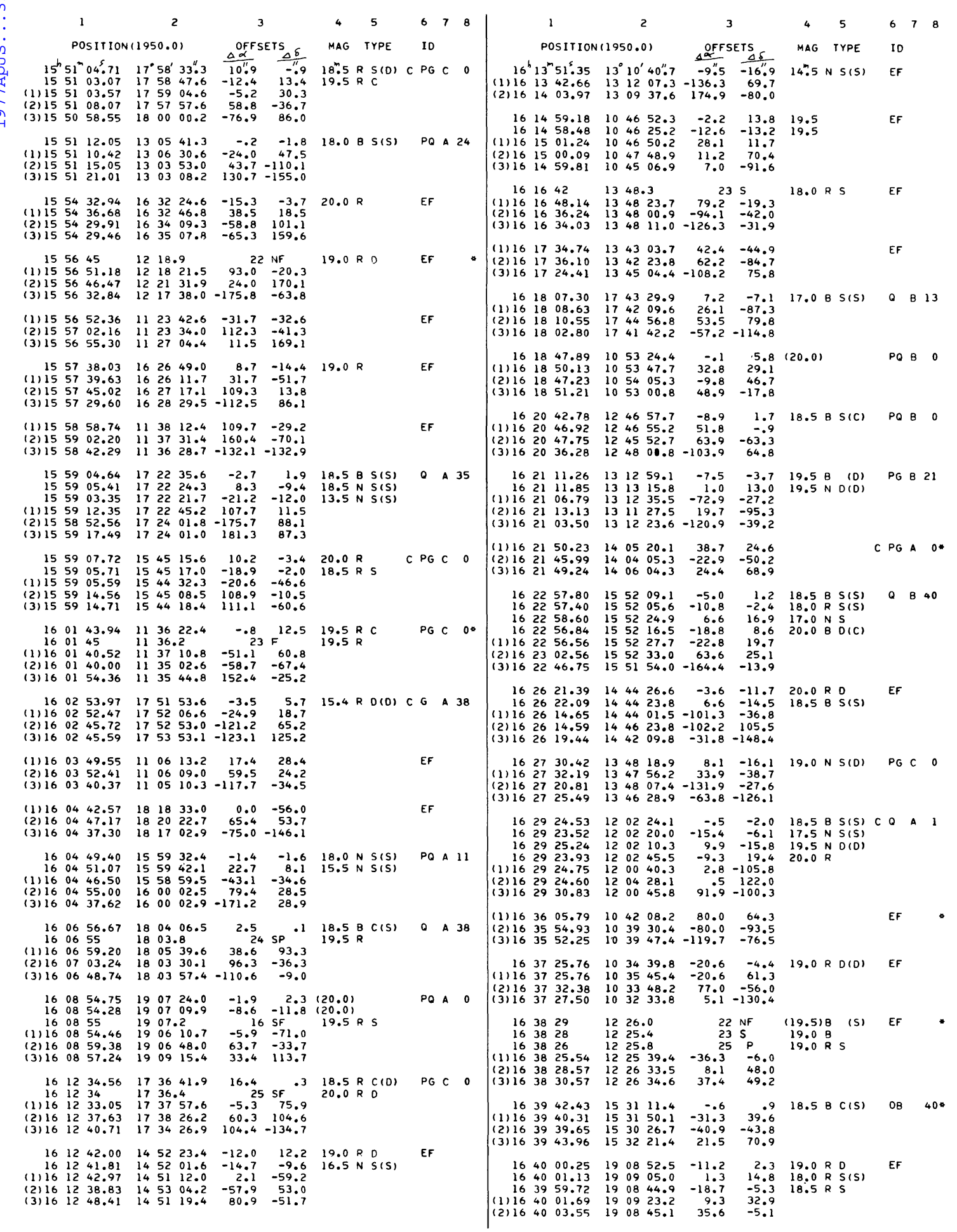


TABLE 3-Continued

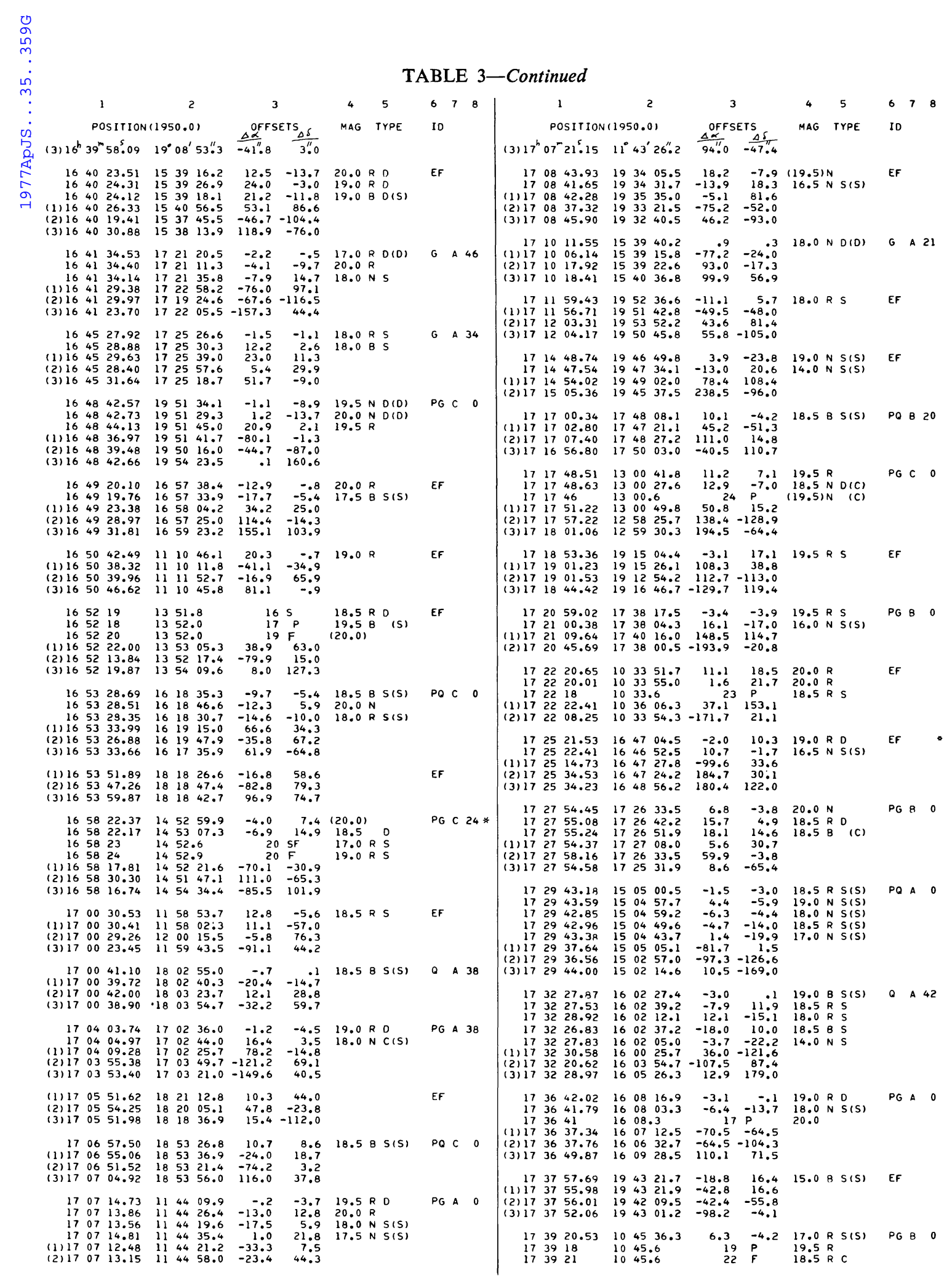


TABLE 3-Continued

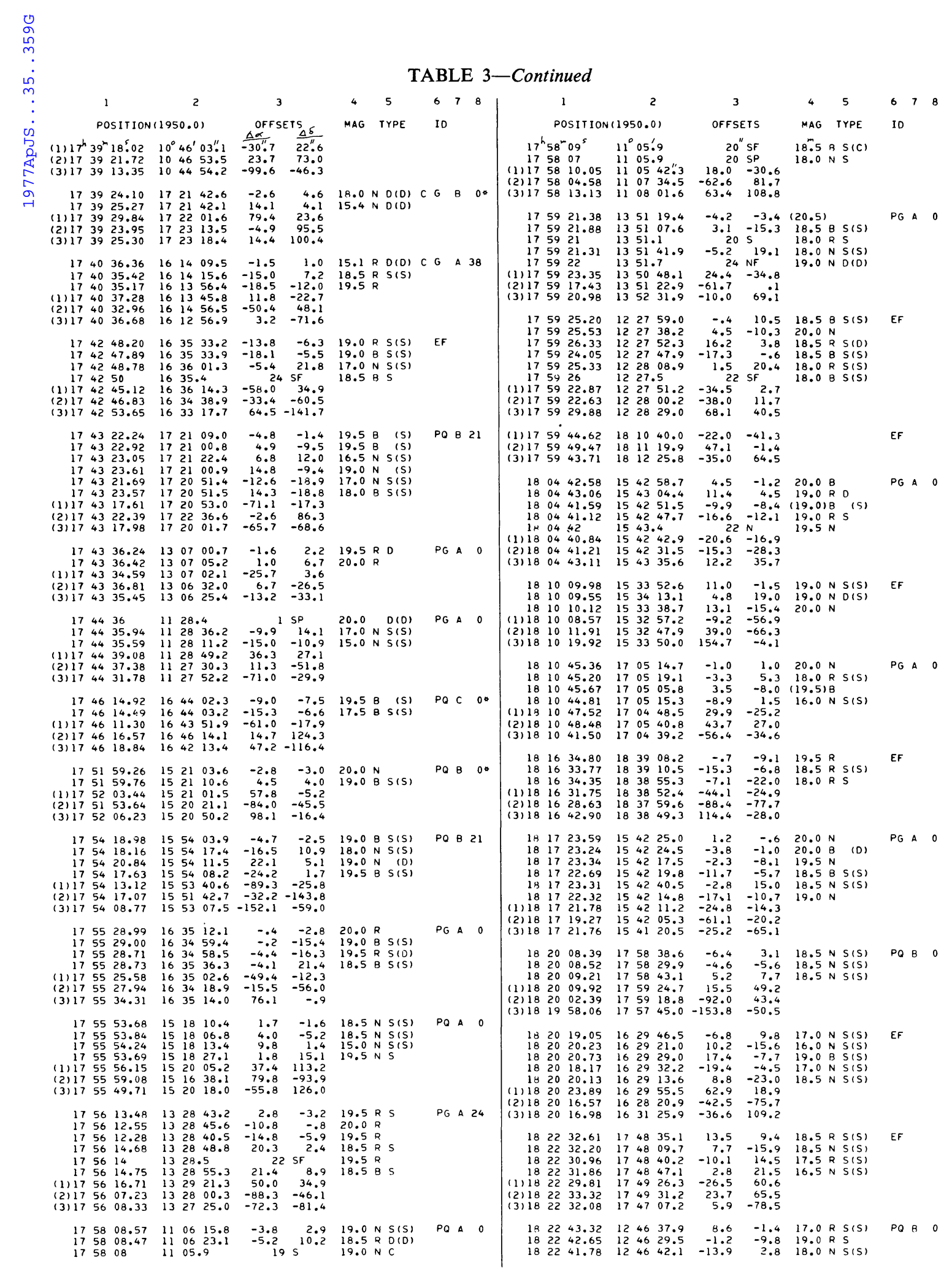


TABLE 3-Continued

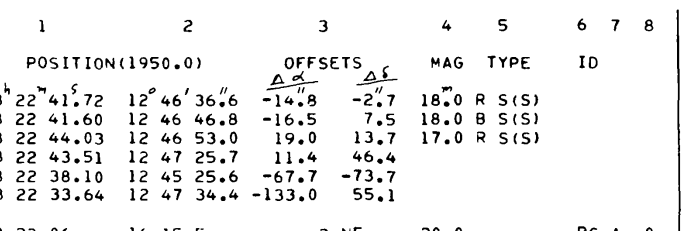

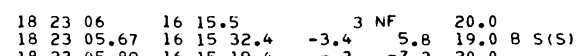

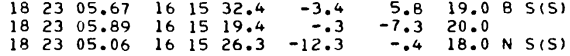

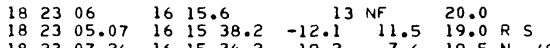

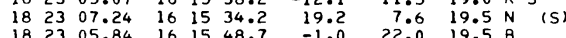

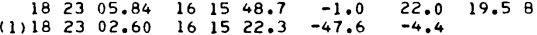

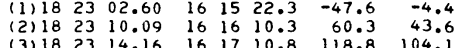

$\begin{array}{lllllllllll}18 & 28 & 55.99 & 17 & 41 & 39.5 & -5.3 & -7.3 & 20.0 & B & (S)\end{array}$

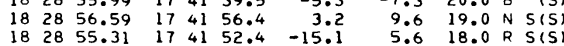

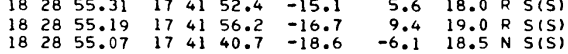

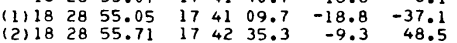

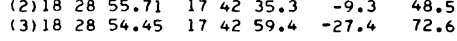

$\begin{array}{llllllllll}20 & 34 & 03.98 & 02 & 46 & 50.5 & 6.1 & 19.4 & 16.0 \mathrm{~N} \mathrm{~S}(\mathrm{~S})\end{array}$

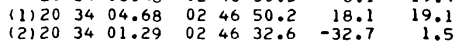

$\begin{array}{lllllllr}\text { (2) } 20 & 34 & 01.29 & 02 & 46 & 32.6 & -32.7 & 1.5 \\ \text { (3) } 20 & 34 & 01.98 & 02 & 46 & 59.0 & -22.3 & 27.9\end{array}$

$\begin{array}{llllllllll}20 & 34 & 24.54 & 03 & 59 & 29.1 & 16.2 & .0 & 19.0 & R\end{array} \quad$ EF

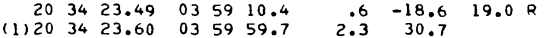
(2) $2034 \quad 15.68 \quad 035930.6-116.3 \quad 1.6$

$\begin{array}{lllllll}20 & 38 & 38.56 & -01 & 22 & 26.0\end{array}$

2038

$\begin{array}{llllll}20 & 38 & 38.53 & -01 & 22 & 35.5\end{array}$

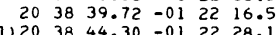

(2) $20 \quad 38 \quad 32.03 \quad-01 \quad 22 \quad 34.0$

(3) $20 \quad 38 \quad 43.31-01 \quad 1954.9$

$\begin{array}{llllll}20 & 42 & 15.85 & 04 & 33 & 06.4 \\ 20 & 42 & 14.77 & 04 & 33 & 05.4\end{array}$

$\begin{array}{llllll}20 & 42 & 16.64 & 04 & 32 & 56.8\end{array}$

$\begin{array}{llllll}20 & 42 & 15.71 & 04 & 33 & 29.5\end{array}$

$\begin{array}{rrrrr}20 & 42 & 14 & 04 & 32.9\end{array}$

$\begin{array}{llllll}\text { (1) } 20 & 42 & 13.68 & 04 & 33 & 44.6 \\ \text { (2) } 20 & 42 & 16.75 & 04 & 32 & 25.3\end{array}$

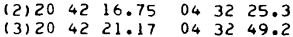

$-27.6-113.3$

$\begin{array}{llll}2.0 & -9.2 & 19.5 R \quad \text { R }\end{array}$

$\begin{array}{rrr}-.4 & 11.8 & (19.5) \mathrm{B} \\ 1.6 & -18.8 & 19.5 \\ 8.4 & -19\end{array}$

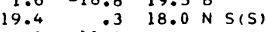

$88.1-11.3$

$\begin{array}{rr}-95.9 & -17.3 \\ 73.3 & 141.8\end{array}$

$\begin{array}{lllll}20 & 42 & 51 & 03 & 13.7 \\ 20 & 42 & 50 & 03 & 13.7\end{array}$

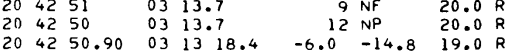

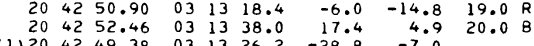

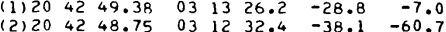

$\begin{array}{llllllll}\text { (2) } 20 & 42 & 48.75 & 03 & 12 & 32.4 & -38.1 & -60.7 \\ \text { (3) } 20 & 42 & 46.82 & 03 & 12 & 48.1 & -67.1 & -45.1\end{array}$

$\begin{array}{lllllll}20 & 45 & 44.33 & 06 & 50 & 09.7\end{array}$

$\begin{array}{llllll}-.3 & -1.1 & 18.0 & R & C(0)\end{array}$

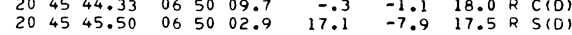

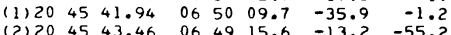

$\begin{array}{llllllll}\text { (2) } 20 & 45 & 43.46 & 06 & 49 & 15.6 & -13.2 & -55.2 \\ \text { (3) } 20 & 45 & 50.99 & 06 & 49 & 26.4 & 98.9 & -44.4\end{array}$

$\begin{array}{llllll}20 & 48 & 55.28 & 07 & 07 & 54.6\end{array}$

204855.420708

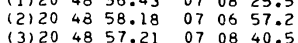

$\begin{array}{llllll}3.1 & 16.2 & 18.0 \mathrm{~N} & \mathrm{~S}(\mathrm{~S}) \quad E F \\ 5.1 & 23.8 & 19.0 & \mathrm{R} & \mathrm{S}\end{array}$

$\begin{array}{rr}50.3 & 47.8 \\ 46.3 & -41.2 \\ 31.8 & 62.1\end{array}$

$\begin{array}{lllllllll}20 & 50 & 48.99 & 05 & 26 & 12.7 & 12.0 & 9.5 & 20.0 R\end{array}$

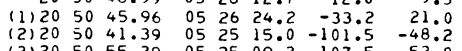

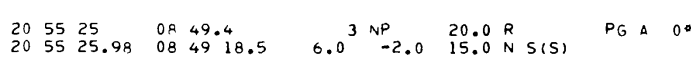

(1) $20 \quad 5528.85 \quad 0850 \quad 07.5 \quad 48.7 \quad 47.0$

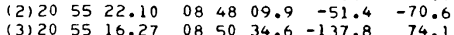

$\begin{array}{lllllllllll}20 & 56 & 01.13 & 05 & 31 & 03.8 & 22.0 & -8.2 & 19.0 & \text { (S) } E F\end{array}$

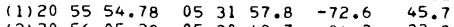

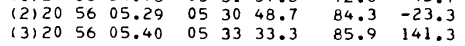

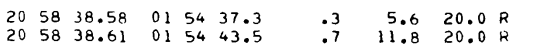

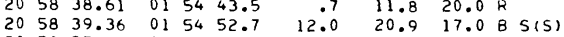

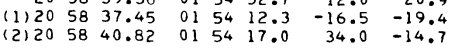

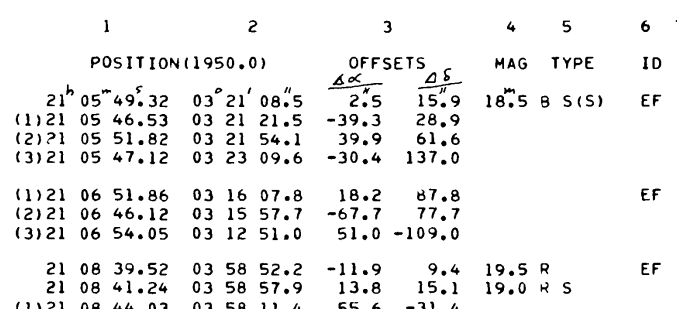

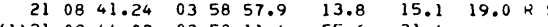

$\begin{array}{llllllrr}\text { (1) } 21 & 08 & 44.03 & 03 & 58 & 11.4 & 55.6 & -31.4 \\ \text { (2) } 21 & 08 & 40.80 & 03 & 59 & 54.8 & 7.3 & 71.9\end{array}$

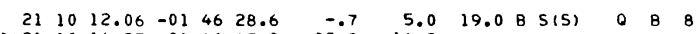

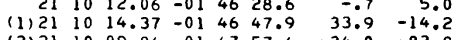

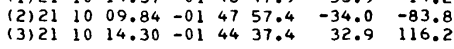

$\begin{array}{rllllllrllll}21 & 11 & 04.68 & 04 & 33 & 50.4 & 11.7 & -1.1 & 18.5 & R & S & E F \\ 21 & 11 & 04.95 & 04 & 33 & 57.4 & 15.8 & 6.0 & 16.5 & N & S(S) & \end{array}$

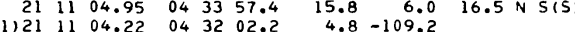

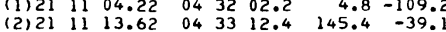

(3) $21 \quad 1101.39 \quad 04 \quad 36 \quad 37.8 \quad-37.4 \quad 166.4$

$\begin{array}{lllllllllll}21 & 15 & 11.50 & 05 & 39 & 24.5 & 6.6 & -11.4 & 18.5 & \mathrm{~N} S & \mathrm{~S} \\ 21 & 15 & 11 & 05 & 39.9 & 21 & \mathrm{NF} & 19.0 \mathrm{R} & \mathrm{S} & \end{array}$

$\begin{array}{llllllll}\text { (1) } 21 & 15 & 08.72 & 05 & 40 & 26.5 & -34.9 & 50.7\end{array}$

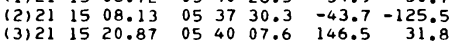

$\begin{array}{lllllllllllll}21 & 20 & 47.06 & 09 & 55 & 01.9 & -2.7 & 2.4 & 19.0 & 8 & S(S) & 0 & 4\end{array}$

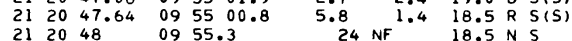

(1) $2122048.66 \quad 0955 \quad 17.3 \quad 20.9 \quad 17.9$

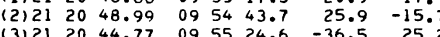

$\begin{array}{lllllll}21 & 2050 & 04 & 27.3 & 17 \mathrm{~N} & (19.0) \mathrm{B} & \text { (S) EF }\end{array}$

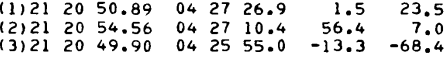

$\begin{array}{rrrrrrrrr}21 & 21 & 11.59 & 02 & 51 & 52.2 & 4.1 & 2.2 & 19.0\end{array}$

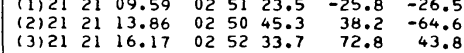

$\begin{array}{llllllll}21 & 2257 & 0515.8 & 4 & 5 P & 20.0 & R & 0\end{array}$

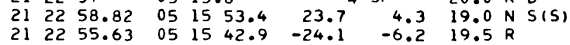

$\begin{array}{llllllll}(1) 21 & 22 & 56.41 & 05 & 14 & 47.1 & -12.3 & -62.0\end{array}$

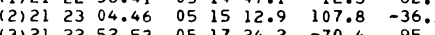

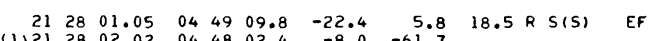

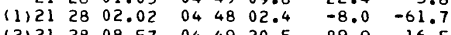

$\begin{array}{llllllrr}(2) 21 & 28 & 08.57 & 04 & 49 & 20.5 & 89.9 & 16.5 \\ 13121 & 27 & 52.14 & 04 & 48 & 34.4 & -155.6 & -29.7\end{array}$

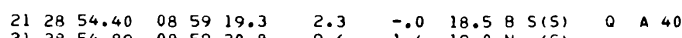

(1) $212853.82090010 .2 \quad-6.3 \quad 50.8 \quad 9.0 \mathrm{~N}$ (S)

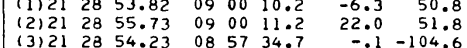

$\begin{array}{llllllrrrrr}21 & 33 & 19.57 & 01 & 04 & 48.3 & -1.2 & 2.9 & 19.0 & 8 & \text { (5) } \\ 21 & 33 & 19.71 & 01 & 04 & 30.8 & 1.0 & -14.6 & 19.5 & R & \end{array}$

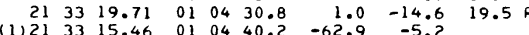

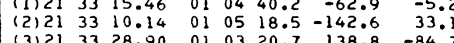

$\begin{array}{lllllllllllll}21 & 34 & 05.21 & 00 & 28 & 25.0 & 2.2 & -.9 & 17.0 & \text { B } & S(5) & 0 & \text { A } 36\end{array}$

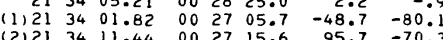

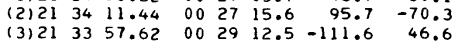

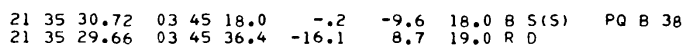

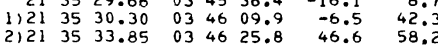

$\begin{array}{rrrrrrrrrrrr}21 & 39 & 39.88 & 02 & 48 & 31.7 & 2.3 & -13.2 & 19.0 & R & S & E F\end{array}$

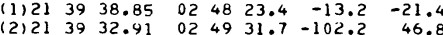

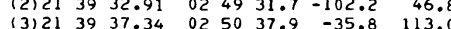

$\begin{array}{llllllllll}21 & 42 & 40.30 & 07 & 46 & 30.5 & 13.2 & -1.0 & 20.0 & R\end{array}$ EF *

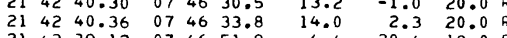

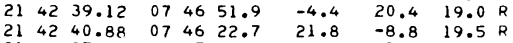

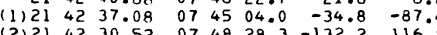

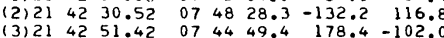

$\begin{array}{llllllll}(1) 21 & 42 & 48.77 & 04 & 17 & 37.9 & 26.7 & -2.8\end{array}$ 
TABLE 3-Continued

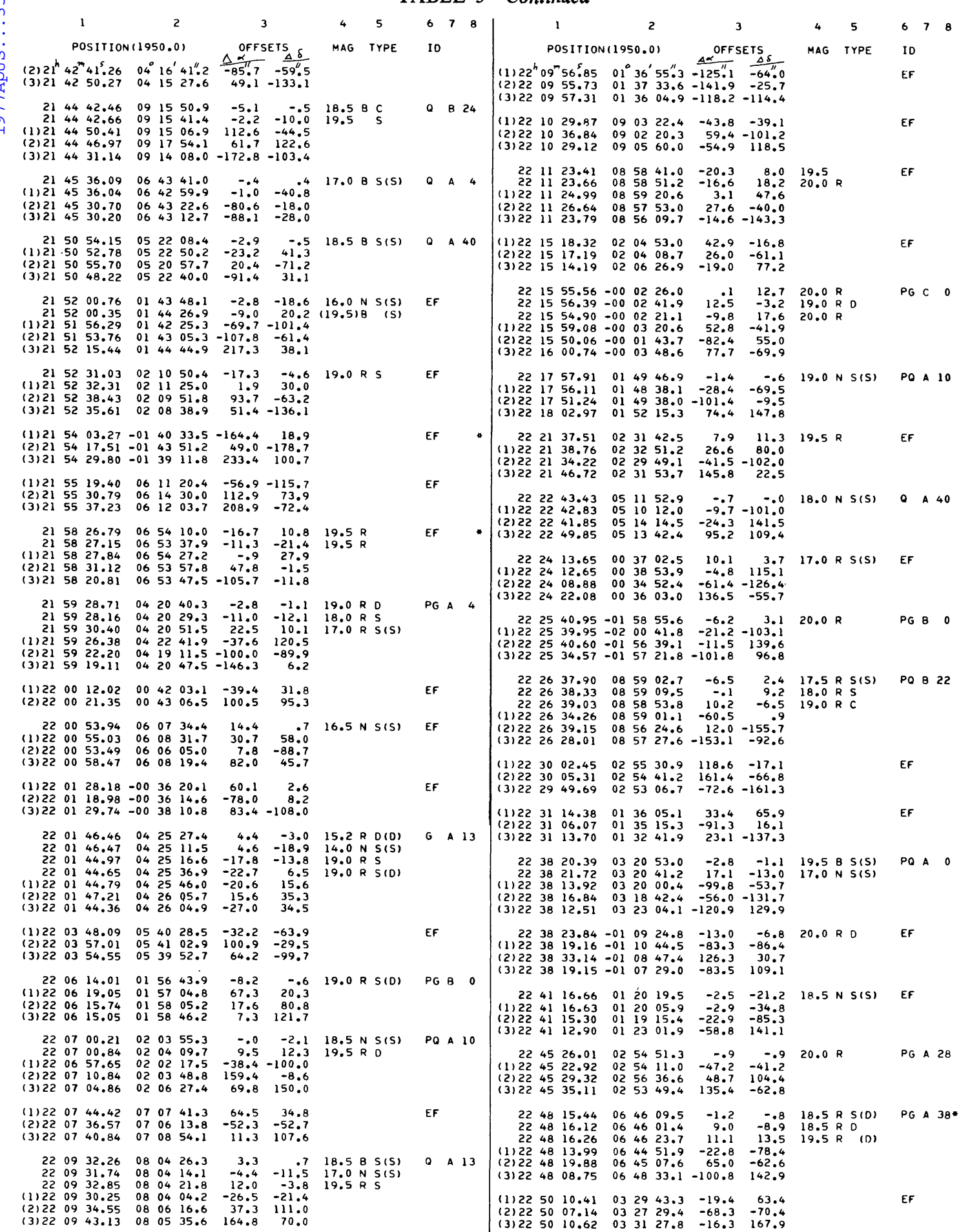


TABLE 3-Continued

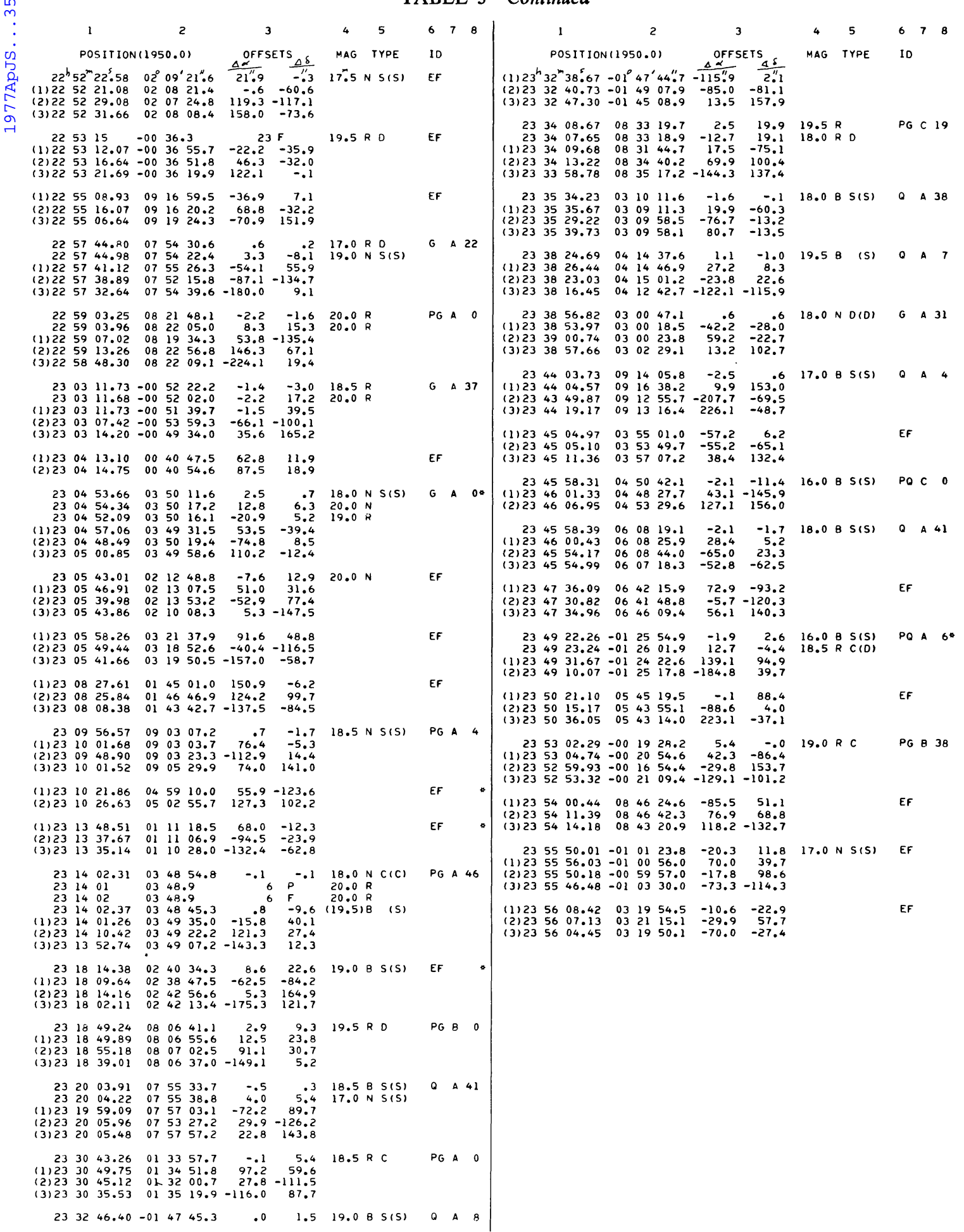




\section{REFERENCES FOR TABLE 3} 1 BALDWIN, J.A., BURBIDGE, E.M., HAZARL, C., MUROOCH, H. S., 185.739

BARBIERI,C. AND BERTOLA,F. 1972,MONTHLY NOTICES ROY. ASTRON. SOC. 156.399.

3 BLAKE,G.M. I970,ASTROPHYS. LFTTERS 6.201.

BOLTON,J.G. AND EKERS,J. 1966, AUSTRALIAN J. PHYS. 19,471 .

BOLTON,J.G. AND EKERS,J. 1966, AUSTRALIAN J. PHYS. 19.559 .

BOLTON,J.G. AND EKERS,J. 1966, AUSTRALIAN J. PHYS 19.713.

BOLTON,J.G.,SHIMMINS,A.J. AND MERKELIJN,J. 1908 , AUSTRALIAN J. PHYS. 21,81 .

8 BOLTON,J.G. AND WALL,J.V. 1970.AUSTRALIAN J. PHYS.

$23,789$.
BROWNE, I.W.A. 1971, NATURE 231.515

BROWNE, I.W.A. . BENTLEY,M. . HAVES,P., MCEWAN,N.J., SPENCER,R.E. AND STANNARD,D.1974,NATUKE 252,209. BROWNE, I.W.A.,CROWTHER,J.H. AND ADGIE,R.L.1973 NATURE 244,146 .

2 BROWNE,I.W.A. ANO MCEWAN,N.J. 1973,MONTHLY NOTICES ROY. ASTRON. SOC.162, $21 \mathrm{P}$.

CLARKE,M.E.,BOLTON,J.G. ANO SHIMMINS,A.J. 1966 AUSTRALIAN J. PHYS. 19,375.

14 COLLA.G.FANTI, C., FANTI, R.,GIOIA, I.,LAKI, C., LEQUEUX ,J.,LUCAS,R. AND ULRICH,M.H. 1975, ASTRON. ASTROPHYS. SUPPL. 20,1 .

15 FANTI,C.,FANTI, R., FICARRA, A., FORMIGGINI, L., GIOVANNI G.,LARI,C. AND PAORIELLI,L. 1975, ASTRON. ASTROPHYS. SUPPL. 19.143

6 GRUEFF, G. AND VIGOTII, M. 1972,ASTRON. ASTROPHYS. SUPPL. 6,1 .

GRUEFF,G. AND VIGOTTI, M. 1975, ASTRON. ASTROPHYS. SUPPL. 20,57

HAZARD, C.,GUIKIS,S. AND SUTTON,J. 1968, ASTROPHYS.

HAZARD, C., JAUNCEY, I.L. AND BACKER,D.C. 1970.ASTRON. J. 75,1039

20 HOSKINS,D.G.,MURDOCH,H.S., ADGIE, R.L., CRO,THER,J.H. AND GENT,H. 1974, MONTHLY NOTICES ROY. $\angle S T R O N$. SOC. 166.235 .

21 HOSKINS,D,G, MURDOCH,H,S, HAZARD,C. AND JAUNCEY,D, L. 1972, AUSTRALIAN J.PHYS. $25,559$.

\section{b) Hand-measured Positions}

Some faint objects within the $25^{\prime \prime}$ radius circles were missed during the plate measuring with the twocoordinate machine. To provide finding charts, all fields were photographed with a camera mounted on the measuring machine at two enlarged scales, 0".62 $\mathrm{mm}^{-1}$ and $4^{\prime \prime} .8 \mathrm{~mm}^{-1}$. The photographs were examined to verify the objects measured on the machine and to see if any objects had been missed. Likely identifications which had been missed were measured on the machine. For other objects which had been missed in the first measuring session, crude positions $\left( \pm 1^{\prime \prime}\right.$ to $\left.\pm 2^{\prime \prime}\right)$ were measured on the $0.62 \mathrm{~mm}^{-1}$ prints using a plastic millimeter scale. Only the distance from the radio source and the octant were recorded, because only the radial distribution of objects was to be considered in the analysis in $\S$ VIII.

\section{c) Magnitudes, Colors, and Morphological Types}

Magnitudes (approximately $m_{v}$ ), colors, and morphological types were estimated by eye for all objects from the $0.62 \mathrm{~mm}^{-1}$ and $4.8 \mathrm{~mm}^{-1}$ photographs of both color PSS plates. For stellar objects visible on the red plates the visual magnitudes are accurate to about \pm 0.5 to $\pm 1.0 \mathrm{mag}$. Magnitudes estimated from the blue plates are less accurate, as are magnitudes of galaxies. For Zwicky (1961-1968) galaxies, Zwicky's magnitude is listed in Table 3.

A color class of red (R), neutral $(N)$, or blue (B) was assigned by comparing the relative sizes of the red-
22 HUNSTEAD,R,W. AND JAUNCEY,D,L, 1970, MONTHLY NOTICES ROY. ASTRON. SOC.149,91.

23 JAUNCEY,D.L. AND HAZARD, C. 1970 , ASTROPHYS. LETTERS 7.1 .

24 JOHNSON,K.H. 1974,ASTRON, J, 79,1006.

25 KRISTIAN $J . A N D$ SANDAGE, i KRISTI

26 KRISTIAN, J.SANDAGE, A. AND KATEM,B, 1974, ASTROPHYS KRISTIAN, J.SA 191,43

7 LANG,K.R.,SUTTON,J.,HAZARD,C. AND GULKIS,S. 1970 , ASTROPHYS. J. 160,17

28 MC EWAN,N.J.,BROWNE, I.W.A. AND CROWTHER, J.H. 1975 MEM. ROY. ASTRON. SOC.80,1

9 MERKELIJN,J.K. 1968, AUSTRALIAN J. PHYS. 21.903.

30 MERKELIJN,J.K., SHIMMINS,A.J. AND BOLTON,J.G. 1968, AUSTRALIAN J. PHYS. $21,523$.

31 MERKELIJN,J.K. AND WALL,J.V. 1971,AUSTRALIAN J. PHYS. 23,575.

32 OLSEN,E.T. 1970,ASTRON. J. 75,764.

33 PENSTON,M,J.,PENSTON,M.V. AND SANDAGE, A. 1971 , PUBL. ASTRON. SOC. PACIFIC, 83,783 .

34 RADIVICH,M.M. AND KRAUS,J.D. 1971, ASTRON. J. $76,643$.

35 SHIMMINS,A.J.,BOLTON,J.G. ANO WALL,J.V. 1975,

AUSTRALIAN J. PHYS.ASTROPHYS. SUPPL, 34,63

6 SHIMMINS, A.J., SEARLE,L., ANDREW,B.H. AND BRANDIE, G. W. 1968, ASTROPHYS. LETTERS 1,167.

37 WALL,J.V. 1971, AUSTRALIAN J. PHYS. ASTROPHYS. SUPPL. 20,1 .

38 WILLS,R.J. 1976, ASTRON. J. 81,1031.

39 WILLS,B.J.,WILLS,D. AND DOUGLAS,J.N. 1973,ASTROV. 1. 78.521 .

40 WILLS,D. AND BOLTON,J.G. 1969, AUSTRALIAN J. PHYS. 22.775 .

41 WILLS D. ANO WILLS B.J. 1974,ASTROPHYS. J. 190,271

42 WILLS.D. AND WILLS.B.J. 1976,ASTROPHYS. J. SUPPL.

SER. 31, 143 .

43 WILLSON,M.A.G. 1970,MONTHLY NOTICES ROY. ASTRON.

SOC $151,1$.

44 WILLSON,M.A.G. 1972,MONTHLY NOTICES ROY. ASTRON.

SOIS. 156,7

45 WYNDHAM,J.D. 1965,ASTRON, $70,384$.

46 WYNDHAM,J.D. 1966,ASTROPHYS. J. $144,459$.

plate and blue-plate images of an object, taking into account zero-point shifts between plates by comparisons of several nearby stars on the two plates.

Morphological classes of stellar (S), compact (C), or diffuse (D) were assigned, using the photographs. Obvious galaxies were called diffuse. In some cases the red-plate morphology was different from that on the blue-plate, so both are given in Table 3. Although colors and morphologies are given for virtually all objects in the table, these classes are probably meaningless for objects with $m_{v} \geq 19.5$.

\section{d) Identification Method}

An identification was suggested whenever an object occurred within $10^{\prime \prime}$ of a radio source. If the nearest object was further than $10^{\prime \prime}$, other criteria were used. Stellar objects further than $10^{\prime \prime}$ were usually not suggested unless bright or blue. Bright galaxies were usually taken to be the identification. Faint galaxies further than $10^{\prime \prime}$ were considered if the radio angular extent appeared large. If no nearby object appeared to be a likely identification, the source is listed as an empty field (EF). However, in many cases listed as $E F$, there are objects within $25^{\prime \prime}$. Future work on the brightness distribution of these sources may show that some of the EFs have visible identifications.

Quasars and BL Lacertae types with confirming spectra are labeled $Q$. Definite stellar objects without known spectra are "PQ" (probable quasars). Faint objects of uncertain morphology are called "PQ" if 
they appear bluish. Galaxies clearly discernible as such are labeled "G." Faint identifications which do not appear very stellar or bluish are called "PG" (probable galaxy). A few very faint objects are listed simply as "O" (object).

\section{e) Explanation of the Optical Data Table}

Optical positions, magnitudes, colors, and morphological types are given in Table 3 for all optical objects found within $25^{\prime \prime}$ of 664 radio sources. If more than one object occurs within $25^{\prime \prime}$ they are listed in order of increasing radio-optical displacement. The identification is generally the closest object. If not, the situation is explained in the notes $(\S \mathrm{Vg})$.

Identification charts, reproduced from the PSS plates, are given in Figure 2 (Plates 29-36) for all suggested identifications having no previously published chart. In a few cases of disagreement with a previously published identification, a chart showing the new identification is given. No charts are given for empty fields.

Secondary position standards are listed for each field. Their positions are preceded by a number in parentheses which identifies them on the chart.

References to previously published charts are given in the References to Table 3. The notes to the table $(\S \mathrm{Vg})$ mention particularly interesting objects, disagreements with previous identifications, and additional references. More references may be found in the compilation by Véron and Véron (1974) and its updates (privately circulated).

The key to Table 3 is as follows:

Columns (1) and (2): Optical right ascension and declination (1950.0). A number in parentheses preceding a position is the index number of a secondary position standard which identifies it on the chart, if one appears in Figure 2.

Column 3: The offsets $\Delta \alpha$ and $\Delta \delta$ (both in arc seconds) from the radio positions (given in Table 2; Douglas et al. [1973], Ghigo and Owen [1973], or Sharp and Bash [1975]), in the sense optical minus radio. If the optical position of an object was not determined by the least-squares fit, but from hand measurements on the $0.62 \mathrm{~mm}^{-1}$ prints, the approximate position is listed in columns (1) and (2); column (3) is the radial distance to the radio source in arc seconds (rms accuracy $\pm 1^{\prime \prime}$ to $\pm 2^{\prime \prime}$ ) and the octant in which the optical object lies with respect to the radio source $(\mathrm{N}=$ north, $\mathrm{S}=$ south, $\mathrm{P}=$ preceding, $\mathrm{F}=$ following).

Column (4): $m_{v}$, estimates from the red PSS plate, accuracy about \pm 0.5 to $\pm 1.0 \mathrm{mag}$ (see $\S \mathrm{V} c$ ). If enclosed in parentheses, estimated from the blue plate.

Column (5): A color estimate, $\mathrm{R}=$ red, $\mathrm{N}=$ neutral, $\mathrm{B}=$ blue, and morphological types $\mathrm{S}=$ stellar, $\mathrm{C}=$ compact, and $\mathrm{D}=$ diffuse. The red plate morphological type is given first, followed by the blue plate type in parentheses.
Column (6): A code for the most probable identification (see $\S \mathrm{V} d$ ). The codes are:

$\mathrm{G}$, galaxy;

$\mathrm{Q}$, quasar or BL Lacertae-type, confirmed by optical spectra;

PG, probable galaxy;

$\mathrm{PQ}$, probable quasar or BL Lacertae-type object;

O, faint object;

$>\mathrm{G}$, probable galaxy not visible on the PSS plates, but found by someone else using deeper plates;

$>\mathrm{Q}$, as above, but a possible quasar or BL Lacertaetype object;

$\mathrm{EF}$, empty field;

$\mathrm{OB}$, bright star obscures radio position;

$\mathrm{C}$, cluster or galaxy group in the vicinity (three or more galaxies within $\sim 2^{\prime}$ ) (usually precedes one of the previous codes, e.g., $C P G)$.

Column (7): The reliability class of the identification, based on position agreement. The object's apparent magnitude is not taken into account here, only the radio-optical position difference $(r)$. See $\S$ VIII and Table 7.

A, $r \leq 5^{\prime \prime}$ (reliability is $\geq 90 \%$ );

$\mathrm{B}, 5^{\prime \prime}<r \leq 10^{\prime \prime}$ (reliability is $70 \%$ to $90 \%$ );

$\mathrm{C}, r>10^{\prime \prime}$ (reliability is $<70 \%$ ).

Column (8): The reference to a finding chart. The key to the reference number appears below Table 3 . If zero appears in this column, a chart is given in Figure 2. If an asterisk appears in this column there is a note about this object in $\S \mathrm{Vg}$.

\section{f) Finding Charts}

Charts are given in Figure 2 for the 118 objects listed with a zero in column (8) of Table 3 . In the chart labels, "B" means the chart was copied from the blue, or "O," PSS plate, and " $R$ " means it was copied from the red, or " $E$," plate. The reticle lines mark the radio source position. They do not necessarily mark a visible object. Index numbers of secondary position standards are indicated. Lines are drawn to indicate secondary position standards when there might be some doubt as to which object is meant.

\section{g) Comments on Individual Identifications}

The literature search on which these notes are based is reasonably complete to mid-1976.

$0038+0973$ C 18 .- - The $18.5 \mathrm{mag}$ galaxy $24^{\prime \prime} \mathrm{N}$ was suggested by Wyndham (1966). Since Fomalont and Moffet (1971) give an angular size of $17^{\prime \prime}$ EW by $41^{\prime \prime} \mathrm{NS}$, none of the three galaxies within $25^{\prime \prime}$ of the radio source can be ruled out.

$0040+064 .-$ Galaxy suggested by Merkelijn (1969) is $50^{\prime \prime} \mathrm{N}$. There is a 20 th mag probable galaxy $3^{\prime \prime}$ from the radio source position, also found by Wills (1976).

$0043+000$.-Galaxy given by Merkelijn and Wall (1970) is $30^{\prime \prime} \mathrm{P}$ and $15^{\prime \prime} \mathrm{S}$ of the UTRAO radio position. $0055+063$. - A 16th mag star $4^{\prime \prime}$ from radio source position has a stellar spectrum (Wills and Wills 1976), and may be obscuring the true identification.

$0059+017$. - A very faint image occurs on the red plate within $3^{\prime \prime}$ of the radio source. This may well be the identification. 
$0103+062$. - The 17 th mag stellar object has a diffuse jet extending NF, at similar position angle to radio structure double model fitted by Cotton, Owen, and Ghigo (1975).

$0114+074$. - This source is not the object suggested by Clarke, Bolton, and Shimmins (1966) and found to have $z=0.861$ by Bolton (Agnew and Arp 1973).

0115-016.- The UTRAO radio position is $35^{\prime \prime} \mathrm{SF}$ the galaxy suggested by Bolton and Ekers (1967). McEwan, Browne, and Crowther (1975) also find EF.

0118-001.-A $13.5 \mathrm{mag}$ object found to have stellar spectrum (Wills and Wills 1974; Strittmatter et al. 1974).

$0118+034$. The quasar has $z=0.765$ (Bolton and Wall 1970). McEwan, Browne, and Crowther (1975) find an offset of $2 " .8 \mathrm{P}$ and $5 " .2 \mathrm{~S}$, in disagreement with our radio-optical offsets. Macdonald and Miley (1971) give complex structure, $35^{\prime \prime}$ diameter.

$0128+040$.-Fomalont (1971) gives 359" double, P.A. $=31^{\circ}$. A 19th mag galaxy $11^{\prime \prime} \mathrm{P}, 12^{\prime \prime} \mathrm{N}$ may be the identification.

$0805+269$. - Recent observations by the author with the $\mathrm{NRAO}^{2}$ interferometer show that the Ghigo and Owen (1973) position is lobe-shifted. Thus the information listed for this source in Table 3 is irrelevant. Two sources were found in this vicinity. One is a $20^{\prime \prime}$ double at P.A. $=160^{\circ}$ and having $S_{2695}=$ 122(12) $\mathrm{mJy}$. Its centroid position (epoch 1950.0) is $08^{\mathrm{h}} 05^{\mathrm{m}} 01^{\mathrm{s}} .81(0 \mathrm{~s} 04),+27^{\circ} 01^{\prime} 28^{\prime \prime} .8\left(00^{\prime \prime} 8\right)$. The identification is a 19th mag probable galaxy within $1^{\prime \prime} \pm 1^{\prime \prime}$ of the radio centroid, in a faint cluster. The other source appears to be single, with $S_{2695}=168(11) \mathrm{mJy}$. Its position (epoch 1950.0) is $08^{\mathrm{h}} 05^{\mathrm{m}} 34 \mathrm{~s} 28(0 \mathrm{~s} .02)$, $+26^{\circ}$ $55^{\prime} 24^{\prime \prime} 3\left(0^{\prime \prime} .2\right)$. The identification seems to be a 20 th mag object, but I have not measured its optical position.

$0807+276$. - The BSO 35" NF the position was suggested by Grueff and Vigotti (1973) and Fanti et al. (1975).

$0817+307$. - "Dumbbell" galaxy. The radio position coincides with the NF component. Position angle of galaxy and radio structure model position angle (Cotton, Owen, and Ghigo 1975) coincide (both position angles are about $60^{\circ}$ ).

$0827+235$. - A 20th mag object suggested by Hazard, Gulkis, and Bray (1967) is about 35" NP the UTRAO radio position. There may be a very faint ( $>20$ mag) object within $3^{\prime \prime}$ of our position, visible only on the red plate.

$0828+324$ and $0828+325$. - These seem to have large radio angular structure. They are probably two components of the 16th mag $\mathrm{E}$ galaxy about $1^{\prime} \mathrm{F}$ $0828+324$. Gearhart et al. (1972) suggested a BSO $14^{\prime \prime} \mathrm{S}$ of the E galaxy.

$0829+280$. - Wills and Wills (1976) find an M-type stellar spectrum for this object. It may be obscuring the true identification.

$0838+325$. - Cotton, Owen, and Ghigo (1975) give structure as $15^{\prime \prime}$ double at P.A. $=170^{\circ} \pm 50^{\circ}$. Major axis of galaxy is at P.A. $\approx 140^{\circ}$.

\footnotetext{
2 The National Radio Astronomy Observatory is operated by Associated Universities, Inc., under contract with the National Science Foundation.
}

$0840+299$. - Olsen (1970) gives $18.5 \mathrm{mag}$ object $50^{\prime \prime} \mathrm{S}$, shown by Schmidt (1974) to have a stellar spectrum.

$0844+319$. Nearby object suggested by Olsen (1970) shown to be a quasar by Strittmatter et al. (1974) and Schmidt (1974). Westerbork radio structure (Colla et al. 1975) shows $15.5 \mathrm{mag} \mathrm{E}$ galaxy is the identification, not the quasar.

$0907+245$. - Four faint galaxies in the area. Hazard and Jauncey (1972) suggest the galaxy 23" SF.

$0922+322$. - An 18th mag BSO $16^{\prime \prime} \mathrm{N}$, suggested by Olsen (1970), shown to have stellar spectrum by Schmidt (1974).

$0923+257$. - An 8.5 mag star blots field. Wills and Wills (1974) found this object to have a stellar absorption spectrum.

$0927+252$. - Spectrum by Wills and Wills (1976) is inconclusive, but probably is that of a quasar.

$0932+241 A$. - Source is 0.6 cluster radii from the center of an ED Zwicky cluster.

$0932+241 B$. - Wills (1976) suggests the RSO 9" NF, but there is a $20-21$ mag slightly blue object $7 " \mathrm{SP}$ which may be the identification.

$0943+257$. - A Zwicky galaxy, $15.6 \mathrm{mag}$, is $36^{\prime \prime} \mathrm{F}$, which may be the correct identification, since Cotton, Owen, and Ghigo (1975) find a 60" double model at P.A. $=50^{\circ}$.

$0949+246$ - - Very faint object $6^{\prime \prime}$ NF the NF component of double object noted by Bolton, Shimmins, and Merkelijn (1968) is about $2^{\prime \prime}$ NF the radio position. Since it lies on a diffraction ring of a nearby bright star, its reality is in doubt. This object is not listed in Table 3, but the two objects noted by Bolton, Shimmins, and Merkelijn (1968) are.

$0949+287$. - The declination given by Grueff and Vigotti (1975) appears to contain a typographical error: They give 43 for the minutes of declination in their Table 2, where I found 42.

$0958+256$.-Low-frequency variable: Cotton (1976) found that the $365 \mathrm{MHz}$ flux density decreased from $1.8 \mathrm{Jy}$ to $1.2 \mathrm{Jy}$ during the latter half of 1973 .

$0958+290,3 C$ 234.-Riley and Pooley (1975) find three components of this source. The UTRAO radio position is $15^{\prime \prime} \mathrm{F}$ the extreme SP component. A 17.5 mag galaxy in a group coincides with the central component.

$1047+280$. - There is a 16 th mag stellar object and an 18-19 mag galaxy near the NF lobe-shift position.

$1055+314$. The radio position may be affected by resolution. The right ascension is one lobe $P$ the $B 2$ position, while the declinations agree (not an allowed lobe-shift from the B2 position).

$1057+307$. - The galaxy suggested by Grueff and Vigotti (1975) is $40^{\prime \prime} \mathrm{NP}$ the UTRAO radio position. The B2 position coincides with the galaxy. The UTRAO position is probably affected by resolution.

$1106+252$. - Called an empty field by Wyndham (1966) and Kristian, Sandage, and Katem (1974). The galaxy $16^{\prime \prime} \mathrm{N}$ is a possible identification since Cotton, Owen, and Ghigo (1975) find this to be a $30^{\prime \prime}-40^{\prime \prime}$ double source, P.A. $=45^{\circ} \pm 5^{\circ}$.

$1118+237$. - The wrong object was marked on the 
finding chart given by Kristian and Sandage (1970). The correct object is barely visible on the PSS plates, and is at the radio position (see the erratum in $A p$.J., 205, 308 [1976]).

$1125+260$.- Group of galaxies in this region. NGC 3689 is about $8^{\prime} \mathrm{S}$, noted previously by Caswell and Wills (1967) and Aizu (1966).

$1133+262$. - Wills and Wills (1974) find this object to have a stellar absorption spectrum.

$1202+297$. - The UTRAO radio position is $\sim 1^{\prime}$ SP the galaxy suggested by Grueff and Vigotti (1972).

$1202+267$. - There is an 18 th mag galaxy near the SP lobe-shift position.

$1222+264$. - The radio position is $15^{\prime \prime} \mathrm{F}$ the 18.519.0 mag galaxy suggested by Olsen (1970), but in view of the $35^{\prime \prime}$ angular size given by Cotton, Owen, and Ghigo (1975), it may be the identification.

1235+196.-Shimmins, Bolton, and Wall (1975) say empty field, but there is a $19.5 \mathrm{mag}$ BSO $8^{\prime \prime} \mathrm{P}$.

$1236+327$.-Olsen (1970) suggests $18.5 \mathrm{mag}$ galaxy $\sim 20^{\prime \prime}$ NF. Grueff and Vigotti (1972) say empty field. There is a $20.5-21 \mathrm{mag}$ object at the radio position seen only on the blue plate.

$1237+231$ - - Object suggested as a possible quasar by Hazard, Jauncey, and Backer (1970) is $\sim 40^{\prime \prime}$ SP.

$1238+243$. - There is a faint object within $5^{\prime \prime}$ of the radio source visible on the red plate only.

$1241+254$. - The 16 th mag NSO $15^{\prime \prime} \mathrm{F}$ has a stellar spectrum (Wills and Wills 1974).

$1245+115$.- The 13th mag RSO 5" SF has a stellar spectrum (Wills and Wills 1974).

$1300+320$.- The $18.5 \mathrm{mag}$ galaxy $10^{\prime \prime} \mathrm{P}$ is not the 16th mag galaxy suggested by Grueff and Vigotti (1972).

$1303+192$. - The wrong object was marked on the finding chart of Wills and Bolton (1969) and then was observed and found to be a star by Lynds and Wills (1972). The correct object, which Wills and Bolton meant to mark, is marked on the chart in Figure 2.

$1308+326$. - This had brightened to 17 th mag and was found to have a BL Lacertae-type spectrum in 1976 April by Wills and Wills (Marsden 1976b). H. R. Miller reports it at $B \approx 14$ in 1974 April. On archival Harvard plates, it is usually $>17.5 \mathrm{mag}$, but in the 1940s reached $14.4 \mathrm{mag}$ (Marsden 1976a).

$1319+270$. - Object suggested by Hazard, Jauncey, and Backer (1970) is about 40" NP the radio position.

$1320+325$. - Object suggested by Olsen (1970) is $38^{\prime \prime}$ SP the radio position, and was shown to be a star by Schmidt (1974). There is a faint object within $3^{\prime \prime}$ of the radio position, not visible on the red plate. Grueff and Vigotti (1975) call it neutral.

$1324+230$. - A $17.5 \mathrm{mag}$ galaxy suggested by Hazard and Jauncey (1972) is $18^{\prime \prime} \mathrm{S}$ of the radio position.

1326+ 150.-Hazard, Jauncey, and Backer (1970) give 15.5 mag galaxy $28^{\prime \prime} \mathrm{F}, 49^{\prime \prime} \mathrm{S}$ of the radio position.

$1333+275$. - I disagree with both the $17.5 \mathrm{mag}$ galaxy 50 " S suggested by Olsen (1970) and the 19.5 mag BSO $25^{\prime \prime} \mathrm{S}$ given by Willson (1972).

$1338+288$. - The faint galaxy mentioned by Hazard,
Jauncey, and Backer (1970) may be one of the objects listed in Table 3.

$1338+107$. - Object noted by Wills and Bolton (1969) 60" SF the radio position was found to have a stellar spectrum (Lynds and Wills 1972).

$1339+266$. - VV 5.32.63. Compact group of galaxies.

$1345+245$. - Olsen (1970) suggested a $17.7 \mathrm{mag}$ galaxy $40^{\prime \prime} \mathrm{SP}$.

$1353+186$. - The radio position listed in Ghigo and Owen (1973) is lobe-shifted. This was called to my attention by $\mathrm{D}$. Wills (private communication) too late for correction of Table 3. Recent observations by the author on the four-element interferometer of the National Radio Astronomy Observatory show that the correct position (epoch 1950.0) is $.13^{\mathrm{h}} 53^{\mathrm{m}} 39 \mathrm{~s} 87$ $(0.01),+18^{\circ} 36^{\prime} 57^{\prime \prime} 3\left(0^{\prime \prime} .2\right)$, and that its angular diameter is about 0"3. It coincides with the Seyfert nucleus in the galaxy Markarian 463. Seẽ Adams (1977) for optical data.

$1357+173$. - An $18.5 \mathrm{mag}$ BL Lacertae object $20^{\prime \prime}$ SP found by Baldwin et al. (1973). The closest object to the radio source, a 20th mag probable galaxy $10^{\prime \prime} \mathrm{P}$, is probably the identification. There is evidence of resolution, so the correct identification is not clear.

$1401+123$.-Wills and Wills (1974) find the 16th mag RSO 8" NP to have a stellar absorption spectrum.

$1407+177 .-N G C 5490$ (13.5 mag) is $30^{\prime \prime}$ SF. Very likely to be the correct identification. Cotton, Owen, and Ghigo (1975) give $20^{\prime \prime} \pm 10^{\prime \prime}$ double, P.A. = $150^{\circ} \pm 30^{\circ}$, close to the radio-optical position angle.

$1407+316$.-Position affected by resolution. Grueff and Vigotti (1975) have noted a faint galaxy 70" NP.

$1409+133$. - There may be a very faint, possibly diffuse object within $5^{\prime \prime}$ of the radio position.

$1420+198$. - 3C 300. Optical-radio P.A. $=142^{\circ}$ for the 19.5 mag galaxy $23^{\prime \prime}$ NP. Cotton, Owen, and Ghigo (1975) find radio P.A. $=126^{\circ}$, Macdonald, Kenderdine, and Neville (1968) find P.A. $=135^{\circ}$. The UTRAO radio position centroid agrees with Macdonald et al. (1968).

$1425+267$. - Ton $202(z=0.366,16$ th mag $)$ is $50^{\prime \prime}$ N. Fanti et al. (1975) find three radio components, one coincident with Ton 202 . The position is affected by resolution and agrees with none of the Fanti et al. positions. Reference star (2), given for this field in Table 3, is Ton 202.

$1436+129$.-Both objects within $10^{\prime \prime}$ have stellar spectra (Wills and Wills 1976).

$1454+303$.- Object 9" F has stellar spectrum (Wills and Wills 1974).

$1455+287$. - Olsen (1970) suggests two nearby galaxies, both within $1^{\prime}$ of the radio source. Cotton, Owen, and Ghigo (1975) give structure as 45" double, so they cannot definitely be ruled out.

$1504+163$. - Object mentioned by Wills and Bolton (1969) is $25^{\prime \prime} \mathrm{N}$ and has a stellar spectrum (Wills, private communication). A $17.5 \mathrm{mag}$ BSO is closer: 12 ".6 F, 7".7 S.

$1518+156$. - The $17.5 \mathrm{mag}$ NSO $13^{\prime \prime} \mathrm{N}$ is called a BL Lacertae-type by Baldwin et al. (1973), but D. Wills (private communication) found that it has a stellar spectrum. 
$1521+137$.-Caswell and Wills (1967) suggested the 15.5 mag galaxy $100^{\prime \prime} \mathrm{NF}$.

$1523+161$. - D. Wills (private communication) finds the $18.5 \mathrm{mag}$ BSO $15^{\prime \prime}$ SP to be a star.

$1556+123$. - Cotton, Owen, and Ghigo (1975) find the source to be a $25^{\prime \prime}$ double with P.A. $=40^{\circ}$, which coincides with the direction from the radio position to the 19th mag galaxy $22^{\prime \prime}$ NF.

$1601+116$.-Wills and Bolton (1969) suggested a BSO $45^{\prime \prime} \mathrm{F}$, found to be a star (D. Wills, private communication).

$1621+140$.- There may be a very faint object within $5^{\prime \prime}$ of the radio position visible only on the blue plate in a group of galaxies. There is also an 18th mag peculiar galaxy about $15^{\prime \prime}$ from the SP lobe-shift position.

$1636+106$.- - The object mentioned by Radivich and Kraus (1971) is $80^{\prime \prime} \mathrm{P}$.

$1638+124$. - Object 23" S, given by Wills and Bolton (1969), is a star (D. Wills, private communication).

$1639+155$.- Spectrum of this object by Lynds shows stellar features (D. Wills, private communication).

$1658+148$. - The galaxy $16^{\prime \prime} \mathrm{NP}$ has a diffuse extension in the direction of the radio source. There is also a faint object 8 " NP the radio position.

$1725+167$. - A $16.5 \mathrm{mag}$ NSO 11" $\mathrm{F}$ has stellar spectrum (Wills and Wills 1976).

$1739+173$.-Clarke, Bolton, and Shimmins (1966) suggest the 16th mag galaxy $15^{\prime \prime} \mathrm{NF}$, but the 18th mag galaxy $5^{\prime \prime} \mathrm{NP}$ is more likely.

$1746+167$.-Wills and Bolton (1969) suggest the 16.5 mag galaxy $40^{\prime \prime} \mathrm{N}$.

$1751+153$. - Possible double object. Radio source is on the line joining a 19th mag BSO and a 20th mag object, $10^{\prime \prime}$ apart.

$2042+045$. - A 17th mag BSO found to have a stellar absorption spectrum by Wills and Wills (1974).

$2055+088$. - A 15th mag NSO $\sim 6^{\prime \prime} \mathrm{F}$ has stellar spectrum (Wills and Wills 1976). The identification probably is the very faint galaxy within 3 " of the radio position.

2058+019.- Jauncey and Hazard (1970) suggest the 19 th mag BSO 24" S.

2142+077.- - Jauncey and Hazard (1970) suggest a 17.5 mag BSO $29^{\prime \prime} \mathrm{P}, 96^{\prime \prime} \mathrm{N}$ of the radio position.

$2142+042$. - A 17 th mag BSO $25^{\prime \prime} \mathrm{F}$ noted by Wills and Bolton (1969), found to be a star (D. Wills, private communication).

2154-016.-The radio position may be affected by resolution. The RRE declination (McEwan, Browne, and Crowther 1975) is $1^{\prime} \mathrm{N}$.

$2158+068$. - I disagree with both Gearhart et al. (1972), who suggest a 20th mag galaxy $71^{\prime \prime} \mathrm{SP}$, and with Hazard and Jauncey (1972), who suggest an 18.5 mag galaxy $27^{\prime \prime} \mathrm{N}$.

$2248+067$.-Clarke, Bolton, and Shimmins (1966) suggest the $18.5 \mathrm{mag}$ galaxy $\sim 30^{\prime \prime} \mathrm{S}$.

$2304+038$. - B. and D. Wills (private communication) found the spectrum of this object to be that of an emission-line galaxy, with $z=0.154$.

$2310+050$. - The radio position is probably affected by resolution of this extended radio source, found to be a triple source extending $161^{\prime \prime}$ at P.A. $=72^{\circ}$ (Fomalont 1971). Kristian, Sandage, and Katem (1974) suggest a faint galaxy near the SF lobe-shift position, at the central component of the radio source.

$2313+011$. - The radio position is affected by resolution. Merkelijn (1969) and McEwan, Browne, and Crowther (1975) agree on a radio position $1^{\prime} \mathrm{N}$ of the UTRAO position and on an identification with an 18-20 mag peculiar galaxy. McEwan, Browne, and Crowther (1975) give angular size as 130".

$2318+026$.- - I disagree with the Parkes $2700 \mathrm{MHz}$ position which is $20^{\prime \prime} \mathrm{P}, 22^{\prime \prime} \mathrm{N}$ of the UTRAO radio position. Asymmetric structure (diam. 50") was noted by McEwan, Browne, and Crowther (1975), who suggested an $18.6 \mathrm{mag}$ quasar $6^{\prime \prime} \mathrm{F}, 20^{\prime \prime} \mathrm{N}$ of the UTRAO position, having $z=1.968$ (D. Wills, private communication).

2349-014.-An N galaxy with some quasar-like features, $z=0.174$. See Wills and Wills (1974) for discussion of this object and references.

\section{COMPARISON WITH OTHER ACCURATE OPTICAL POSITIONS}

Table 4 shows the results of comparisons with other accurate optical positions. The position differences are in the sense "this paper minus other." The column headed "Approx. Errors" gives the position errors claimed by the authors of the papers being compared. In all cases, the population standard deviations $\sigma_{\alpha}$ and $\sigma_{\delta}$ are no larger than expected from the combination of the errors quoted in each case with my error estimate of 0.5 to 0.7 .

Only one comparison shows a significant systematic difference. The large difference of $\langle\Delta \alpha\rangle=-1.36 \pm$ 0 ".16 with respect to Véron's (1972) positions is not caused by one or two discrepant cases. All five objects have $\Delta \alpha$ in the range $-1^{\prime \prime}$ to $-2^{\prime \prime}$. A systematic error in the Véron (1972) list seems likely. No such problem is evident in the later list by Véron and Véron (1975), however.

Systematic right ascension differences between different position determinations of small groups of quasars have been noted (Hunstead 1971; Argue and Taylor 1974) and attributed to a possible systematic difference between the SAO and AGK3 systems. Such an effect is not supported by the results in Table 4 . Furthermore, several direct comparisons of SAO and AGK3 positions show no systematic differences (Adgie 1974; Véron and Véron 1975; P. Hemenway, private communication).

\section{BACKGROUND COUNTS}

Objects were counted in a $25^{\prime \prime}$ radius area centered $371^{\prime \prime}$ west of each radio source in the sample. This distance (6.5 BSI lobe shifts) was chosen to allow virtually no lobe-shifted sources to fall in the area, but to have the area close enough to the radio sources that the background counts would be, on the average, the same as in the radio source fields. Since the areas have 
TABLE 4

Optical Position Comparisons (this paper minus other)

\begin{tabular}{|c|c|c|c|c|c|c|c|}
\hline & $\begin{array}{c}\text { Approx. } \\
\text { Errors }\end{array}$ & $\begin{array}{c}\text { Ref. } \\
\text { System }\end{array}$ & $N$ & $\langle\Delta \alpha\rangle$ & $\sigma_{\alpha}$ & $\langle\Delta \delta\rangle$ & $\sigma_{\delta}$ \\
\hline $\begin{array}{l}\text { Wills, Wills, and Douglas } 1973 ; \\
\text { Wills } 1976 \ldots \ldots\end{array}$ & $0 " 4-0.5$ & SAO & 140 & $-0^{\prime \prime} 07+0,05$ & $0 " 55$ & $+0.06+0.05$ & \\
\hline Johnson $1974 \ldots \ldots \ldots \ldots \ldots$ & $0 " 7$ & SA & 19 & $-0.31 \pm 0.23$ & 1.00 & $+0.10 \pm 0 \prime 24$ & $\begin{array}{ll}0 \\
1,05 \\
0.02\end{array}$ \\
\hline Hunstead $1971 \ldots \ldots \ldots \ldots$ & $0 " 4-0 " 5$ & SAO & 7 & $+0.03 \pm 0.08$ & 0,21 & $+0^{\prime \prime} 14 \pm 0 " 12$ & 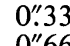 \\
\hline Kristian and Sandage 1970. & $0 " 2-0 " 6$ & AGK3 & 6 & $-0.17 \pm 0.22$ & 0.54 & $-0^{\prime \prime} 18 \pm 0^{\prime \prime} .27$ & $0^{\prime \prime 66}$ \\
\hline Bergamini et al. $1973 \ldots \ldots$. & 1"0 & AGK & 8 & $-0^{\prime \prime} .37 \pm 0.28$ & $0^{\prime \prime \prime}$ & $-0.39 \pm 0.15$ & $\begin{array}{l}0,44 \\
0,93\end{array}$ \\
\hline Véron $1972 \ldots \ldots \ldots \ldots$ & 0.5 & AGK3 & 5 & $-1^{\prime \prime} .36 \pm 0.16$ & $0 " 35$ & $+0^{\prime \prime} 41 \pm 0^{\prime \prime} 42$ & $\begin{array}{l}0,93 \\
0 \prime 68\end{array}$ \\
\hline Véron and Véron 1975. & $0.5-0.6$ & AGK3 & 15 & $-0.23 \pm 0.17$ & 0.65 & $-0.19 \pm 0.18$ & 0.68 \\
\hline
\end{tabular}

virtually the same sky distribution as the radio sources in the sample, these counts are optimum for comparing with counts in the radio source fields.

The counting was done on the $0.62 \mathrm{~mm}^{-1}$ and $4.8 \mathrm{~mm}^{-1}$ photographs mentioned in $\S \mathrm{V} b$. The objects were classified as to color, magnitude, and morphological class as described in $\S \mathrm{V} c$. Counts from both red and blue plates were combined. Objects visible on only one color plate were included in the total counts, so these counts are larger than counts confined to a single color would be.

The total area covered by the background count fields is 362 square arc minutes.

The counts of all objects, stars and galaxies included, to the PSS plate limit are given in Figure 3 as a function of galactic latitude. The error bars represent $\sqrt{ } N$, where $N$ is the number of objects counted in each latitude bin. Some of the points have been slightly shifted horizontally for clarity.

My counts at the north galactic pole are compared in Table 5 with counts by others, and are seen to be in reasonable agreement.

Counts of all objects and of various subclasses are given as a function of galactic longitude and latitude in Table 6. Regions I to VIII of the $l^{\mathrm{II}}-b^{\mathrm{II}}$ plane, referred to in Table 6 , are defined by Figure 4 . The errors given in Table 6 are $\rho / \sqrt{ } N$, where $\rho$ is the surface density and $N$ is the number of objects counted.

\section{COMPLETENESS AND RELIABILITY}

The completeness of an identified sample may be defined as the fraction of the number of visible identifications possessed by the sample that have actually been found. The reliability is the fraction of claimed identifications which are indeed correct. These quantities may be calculated from knowledge of the radio and optical position errors, the size of the search radius, the density of background objects, and the fraction of identifications which are visible. More complete discussions of completeness and reliability and derivations of formulae for calculating these quantities can be found in papers by Condon, Balonek, and Jauncey (1975) and by de Ruiter, Willis, and Arp (1977).

The radius of the area to be searched for identifications is generally taken to be 2 to 3 times the size of the position errors for point sources. For sources of appreciable angular size, one usually increases the search radius in proportion to the angular size. To estimate completeness and reliability, the nominal position errors which go into the calculations are also increased in proportion to the angular sizes of the sources.

Due to the limited spatial frequency coverage of the BSI, accurate angular sizes could not be determined

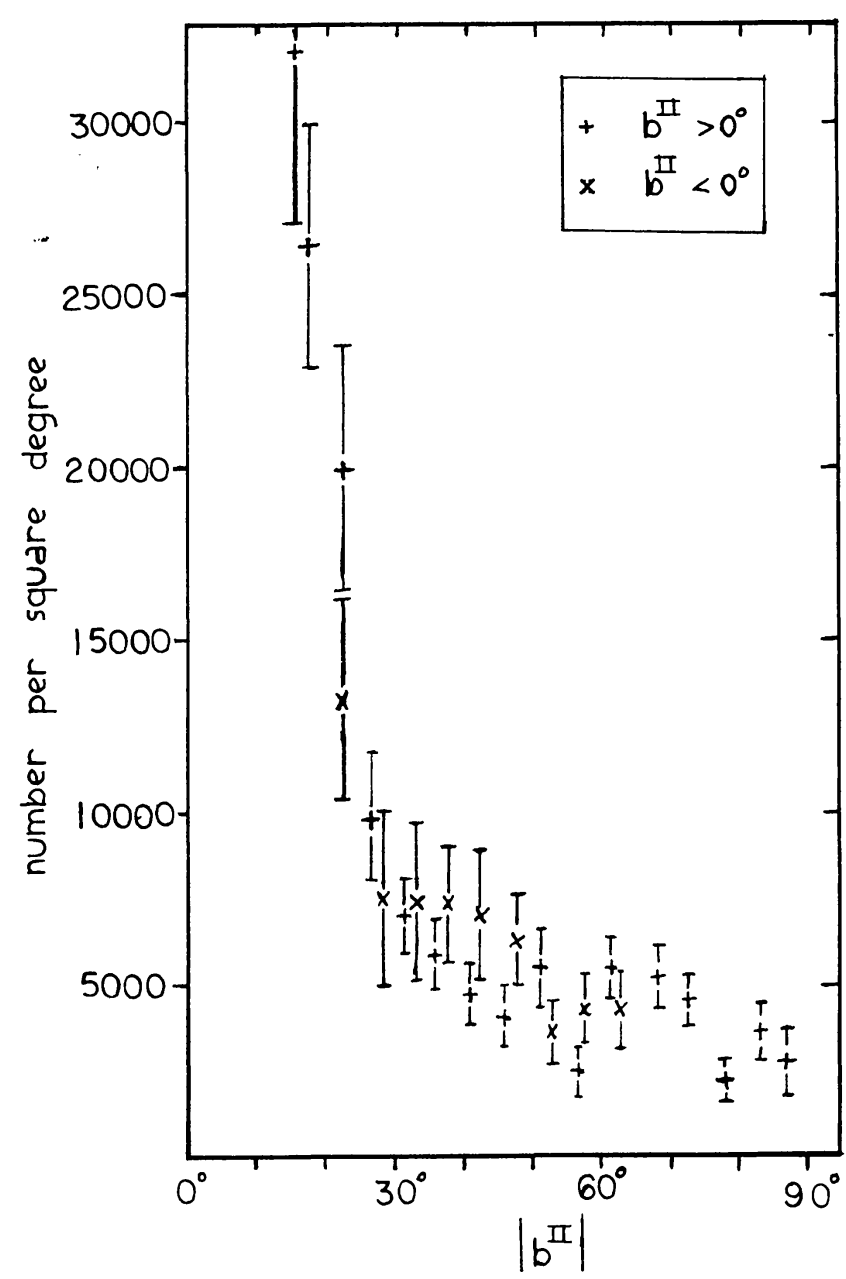

FIG. 3.-Counts of background objects plotted versus galactic latitude. 
TABLE 5

Various Counts in the North Galactic Polar CaP*

\begin{tabular}{|c|c|c|}
\hline Counts by & Objects Counted & $\begin{array}{l}\text { Number per } \\
\text { Sq. Degree }\end{array}$ \\
\hline $\begin{array}{l}\text { Brown } 1973 \ldots \\
\text { Bergamini et al } 1973 \ldots \ldots \\
\text { Braccesi et al. } 1970 \ldots \ldots \ldots \\
\text { This paper. . . . . . . . . }\end{array}$ & $\begin{array}{l}\text { Stars }+ \text { galaxies to } B=21 \\
\text { All objects to PSS print limit } \\
\text { All objects to PSS print limit } \\
\text { All objects to PSS plate limit } 85^{\circ} \leqslant b^{\text {II }} \leq 90^{\circ}\end{array}$ & $\begin{array}{l}2560 \pm 130 \\
3000 \pm 300 \\
2800 \\
2970 \pm 400\end{array}$ \\
\hline
\end{tabular}

* Combining both star and galaxy counts.

for this sample. Nevertheless, the appropriate position error distribution for use in calculating completeness and reliability was determined by the following method: All objects were counted in 1" wide rings centered on the radio sources, out to a radius of $20^{\prime \prime}$. The background count level was subtracted from the resulting distribution, to produce an estimate of the distribution of radio-optical separations for the true identifications. This method works because the background counts are a good representation of the background at the radio sources, as explained in $\S$ VII. This procedure is believed to be freer from bias than using the distribution of radio-optical separations of the identifications found as described in $\S \mathrm{V} d$, since the latter group includes a number of random coincidences.

The number of true identifications estimated by the above method for this sample is $307 \pm 28$. This may be too high since a number of the radio sources probably lie in faint clusters of galaxies. The size of this effect is difficult to assess. Various estimates for this sample suggest that the number of identifications predicted is too high by no more than about 20 , i.e., about $7 \%$. The number of suggested identifications listed in Table 3 is 319 , of which 20 to 30 are probably random coincidences. Thus the number of identifica- tions found by considering individual cases is in approximate agreement with the statistical estimate.

The distribution of radio-optical separations, found as described above, can be fitted reasonably well with a two-component distribution of the following form:

$$
\begin{aligned}
n(r)= & 0.7 \frac{r}{\sigma_{1}{ }^{2}} \exp \left(-\frac{1}{2} r^{2} /{\sigma_{1}}^{2}\right) \\
& +0.3 \frac{r}{\sigma_{2}{ }^{2}} \exp \left(-\frac{1}{2} r^{2} /{\sigma_{2}}^{2}\right),
\end{aligned}
$$

where $n$ is proportional to the number of identifications between $r-1$ and $r$ arc seconds from a radio source, $r$ is the radio-optical separation in arc seconds, $\sigma_{1}=$ $2 " .2$, and $\sigma_{2}=9$ ".5. The quantities $\sigma_{1}$ and $\sigma_{2}$ represent combined radio and optical position errors ; 2 ". 2 is about the expected combined error for point sources.

The interpretation of equation (4) is that $70 \%$ of the identifications (represented by the first term of eq. 4) have no detectable offset of the optical position from the radio source centroid. It is assumed that the appreciable offsets of the remaining $30 \%$ (the second term of eq. 4) are due to these sources having large angular sizes. Such an effect is expected since, in the

TABLE 6

Counts of Objects in Various Regions of the $l^{\text {II }} b^{\text {II }}$ Plane

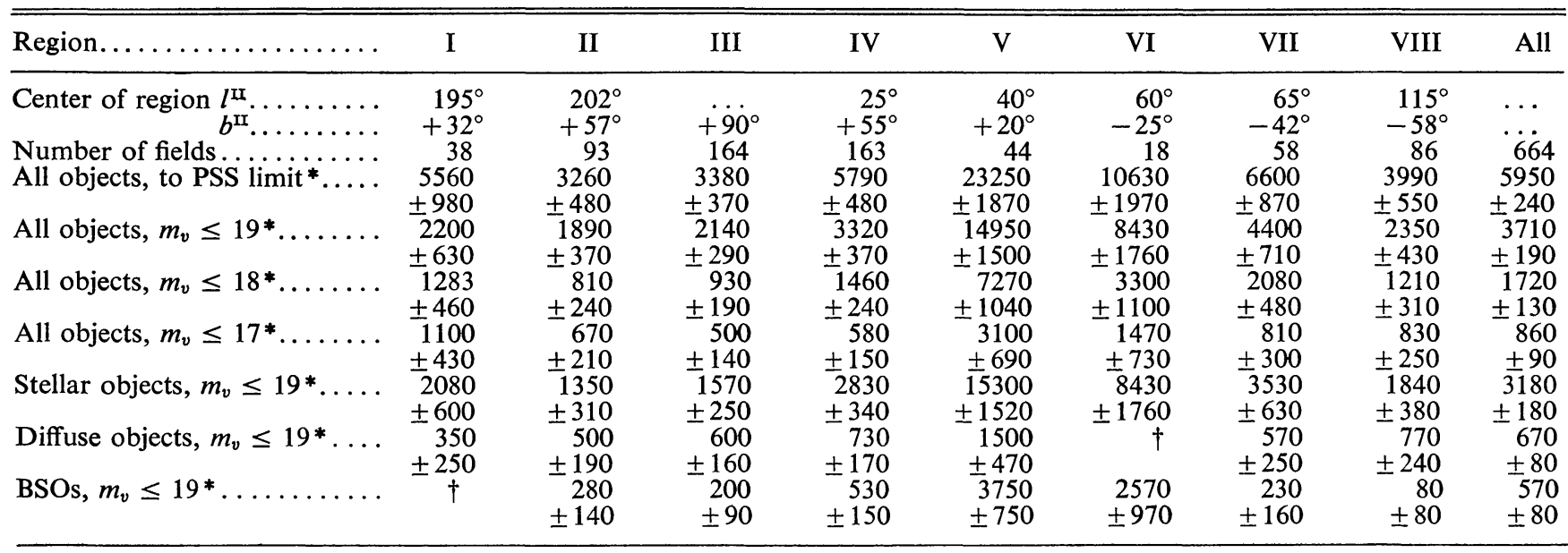

* Densities are given in number per square degree.

$\dagger$ No counts here. 


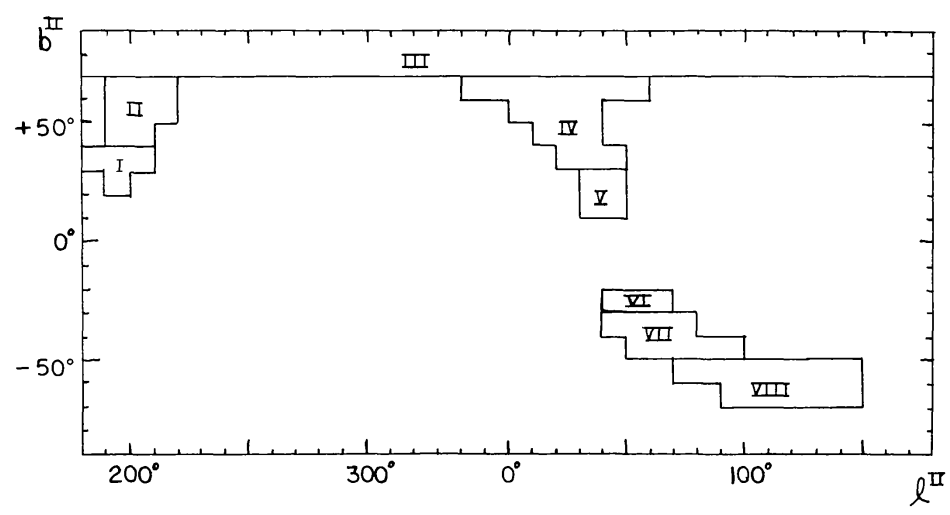

FIG. 4.-Observed regions in galactic coordinates

first place, a measurable difference between the optical and radio centroid may well occur in a source of large angular size, and, second, the BSI, due to its limited spatial frequency coverage, can measure an incorrect radio centroid for an asymmetric brightness distribution having an angular size greater than $10^{\prime \prime}$.

A test was made to see if some of the large radiooptical separations might occur for reasons other than the two outlined above. The 116 radio sources in this sample with no detectable angular extent $\left(\theta \leqslant 15^{\prime \prime}\right)$ were analyzed as to distribution of radio-optical separation. In this subsample, no separations in excess of those expected for point sources were found. Thus no evidence was found for radio-optical offsets resulting from any other than angular size-related causes.

Assuming equation (4) to be the appropriate position error distribution for this sample, and using the derivation of formulae for completeness and reliability described by Condon, Balonek, and Jauncey (1975), the completeness and reliability of these identifications for various search radii was computed, with the results displayed in Table 7. On the basis of these calculations, reliability classes of "A," "B," and "C" were assigned to all suggested identifications as described in the key to Table 3.

Error distributions similar to equation (4) were also determined for the sources identified with galaxies and quasars. In general, galaxies have larger radio angular sizes than quasars. Consistent with this, $\sim 40 \%$ of the galaxies and probable galaxies in the sample were found to have appreciable radio-optical separations $\left(r \geqslant 5^{\prime \prime}\right)$, while only $10 \%$ to $20 \%$ of the quasars and probable quasars exceed separations expected for point sources.

TABLE 7

COMPLETENESS AND RELIABILITY

\begin{tabular}{|c|c|c|}
\hline Search Radius & Completeness & Reliability \\
\hline 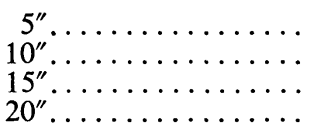 & $\begin{array}{l}0.66 \\
0.80 \\
0.87 \\
0.91\end{array}$ & $\begin{array}{l}0.92 \\
0.81 \\
0.71 \\
0.63\end{array}$ \\
\hline
\end{tabular}

\section{IDENTIFICATION RESULTS}

A tabulation of the numbers of different kinds of identifications is given in Table 8 . The identification classes have the same meaning as in Table 3. From Table 8 , the total number of identifications visible on the PSS plates is 319 . The number of type A identifications $\left(r \leq 5^{\prime \prime}\right.$; reliability $\left.\geq 90 \%\right)$ is given in Table 9 . There are 205 of these, of which about 23 are expected to be spurious, based on an average background rate of $4.758 \times 10^{-4}$ per square arc second.

It is impossible to distinguish between galaxy and stellar images near the plate limit, so statistics of occurrence of these objects are meaningful only above an apparent brightness limit somewhat above the plate limit. For $m_{v} \leq 19.5,102(15 \%)$ of the sources are $G$ or $P G$, and $136(20 \%)$ are $Q$ or $P Q$, having allowed for chance coincidences.

The counts of $G, P G, Q$, and $P Q$ objects by magnitude are shown in Figure 5. The numbers of galaxies increase steadily to the plate limit, but the quasars seem to begin to cut off at a somewhat brighter level, the maximum occurring at $18.5 \mathrm{mag}$. This cutoff in quasar brightness has been noted in many other samples-for example, see Schmidt (1972).

\section{a) Neutral Stellar Objects (NSOs)}

Table 10 gives information for the 15 objects classified as neutral and stellar which lie within $5^{\prime \prime}$ of a

TABLE 8

IDENTIFicATION CONTENT*

\begin{tabular}{|c|c|c|c|}
\hline Class & Number & Class & Number \\
\hline $\begin{array}{l}\mathrm{EF} \ldots \ldots \ldots \\
>\mathrm{G} \ldots \ldots \ldots \\
>\mathrm{Q} \ldots \ldots \ldots \\
\mathrm{C}>\mathrm{G} \ldots \ldots \cdots \\
\mathrm{C} \mathrm{EF} \ldots \ldots \ldots \\
\mathrm{OB} \ldots \ldots \ldots \ldots \\
\mathrm{OB} \ldots \ldots \ldots \ldots\end{array}$ & $\begin{array}{r}306 \\
8 \\
5 \\
2 \\
18 \\
6 \\
3\end{array}$ & $\begin{array}{l}\mathrm{G} \ldots \ldots \ldots \ldots \\
\mathrm{PG} \ldots \ldots \ldots \\
\mathrm{C} G \ldots \ldots \\
\mathrm{C} \mathrm{PG} \ldots \ldots \ldots \\
\mathrm{Q} \ldots \ldots \ldots \\
\mathrm{PQ} \ldots \ldots \ldots \\
\mathrm{CQ} \ldots \ldots \ldots\end{array}$ & $\begin{array}{r}25 \\
104 \\
16 \\
14 \\
79 \\
76 \\
2\end{array}$ \\
\hline
\end{tabular}

* See key to Table 3 for explanation of classes. 

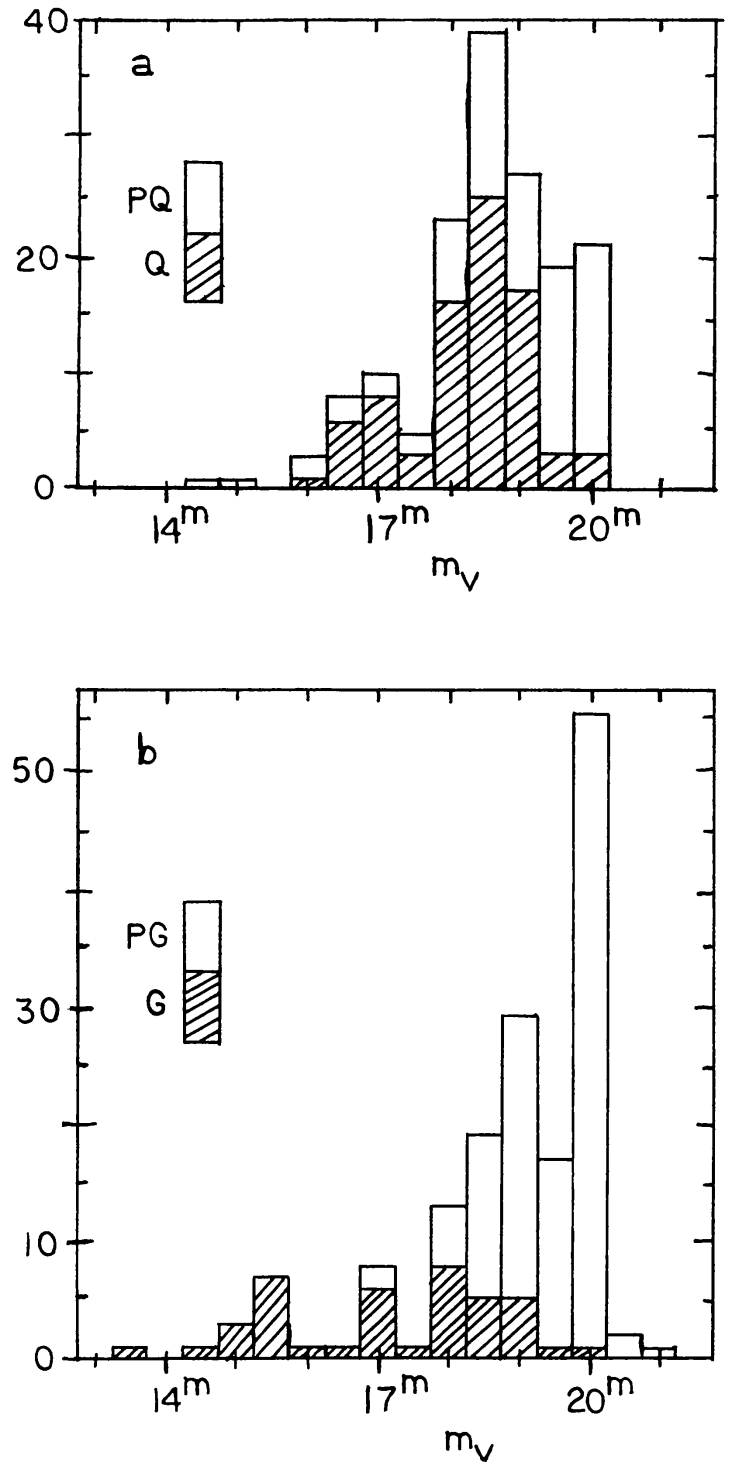

Fig. 5.- Magnitude histograms for the identified objects. (a) Probable and definite quasars or BL Lacertae-type objects. Includes classes $\mathrm{Q}, \mathrm{PQ}$, and $\mathrm{C} \mathrm{Q}$. (b) Probable and definite galaxies. Includes classes $\mathrm{G}, \mathrm{PG}, \mathrm{C}$ G, and C PG.

radio source position. From background counts, 4.7 of them are expected to be chance coincidences.

The spectral indices given in Table 10 were found from this expression:

$$
\alpha \equiv \log \left(S_{1415} / S_{365}\right) / \log (1415 / 365)
$$

The $1415 \mathrm{MHz}$ flux densities from the Ohio catalog were used and were multiplied by the factor 1.18 to put them on the absolute scale of B. J. Wills (1973). The $365 \mathrm{MHz}$ flux densities are mostly total flux densities estimated by fitting brightness distribution models to
TABLE 9

CONTENT OF IDENTIFICATIONS WITHIN 5 ARCSEC

\begin{tabular}{|c|c|}
\hline Classes & Number \\
\hline 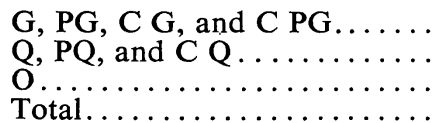 & $\begin{array}{r}95 \\
107 \\
3 \\
205\end{array}$ \\
\hline
\end{tabular}

the Texas data (Bash, Cotton, and Douglas 1974; Cotton, Owen, and Ghigo 1975). Measured total $365 \mathrm{MHz}$ flux densities from the Texas short-baseline survey (Cotton 1974) were used when available. When only the interferometer flux density was available, an upper limit to $\alpha$ is given.

Optical spectra are available for most of these NSOs. They include BL Lacertae types, quasars, and $\mathrm{N}$ galaxies. The prevalence of $\mathrm{BL}$ Lacertae types among flat-spectrum NSOs has been noted, e.g., by Browne, Crowther, and Adgie (1973). Among the confirmed and likely BL Lacertae types in Table 10, the majority have $\alpha>-0.5$, but at least one $(2207+020)$ has a steep spectrum $(\alpha<-0.5)$. Although no confirming spectrum exists for $2217+018$, its lack of UV excess may indicate that it is a BL Lacertae type. It, too, has a steep spectrum.

\section{b) Radio Stars?}

In this sample, 13 objects within $10^{\prime \prime}$ of radio sources were found to have stellar spectra (Wills and Wills 1974; Wills and Wills 1976; C. R. Lynds, private communication). The question may therefore be raised as to whether some of these stars are indeed radio sources. If so, they would be a new class of radio star, since the known types are too faint or too shortlived to be present in this sample.

To answer this question, comparisons with star counts were made. It was found that the expected number of random coincidences, even when confining the attention to radio sources at high galactic latitude, exceeded the number of stars found in this sample. Thus the 13 stars are almost certainly all random coincidences.

I am indebted to Dr. J. N. Douglas for considerable advice and encouragement; to Dr. D. Wills and Dr. B. J. Wills for many enlightening discussions and for allowing me to use some spectroscopic results prior to publication; to Mr. Chip Wolfe for programming; to Dr. P. D. Hemenway and Ms. E. Bozyan for assistance in the plate-measuring project; and to the crew of measuring machine operators. This work was supported by grants from the National Science Foundation and the National Geographic Society, and by the organized research program of the University of Texas. 
TABLE 10

NSOs WITHIN 5 ARCSEc OF a Radio Source

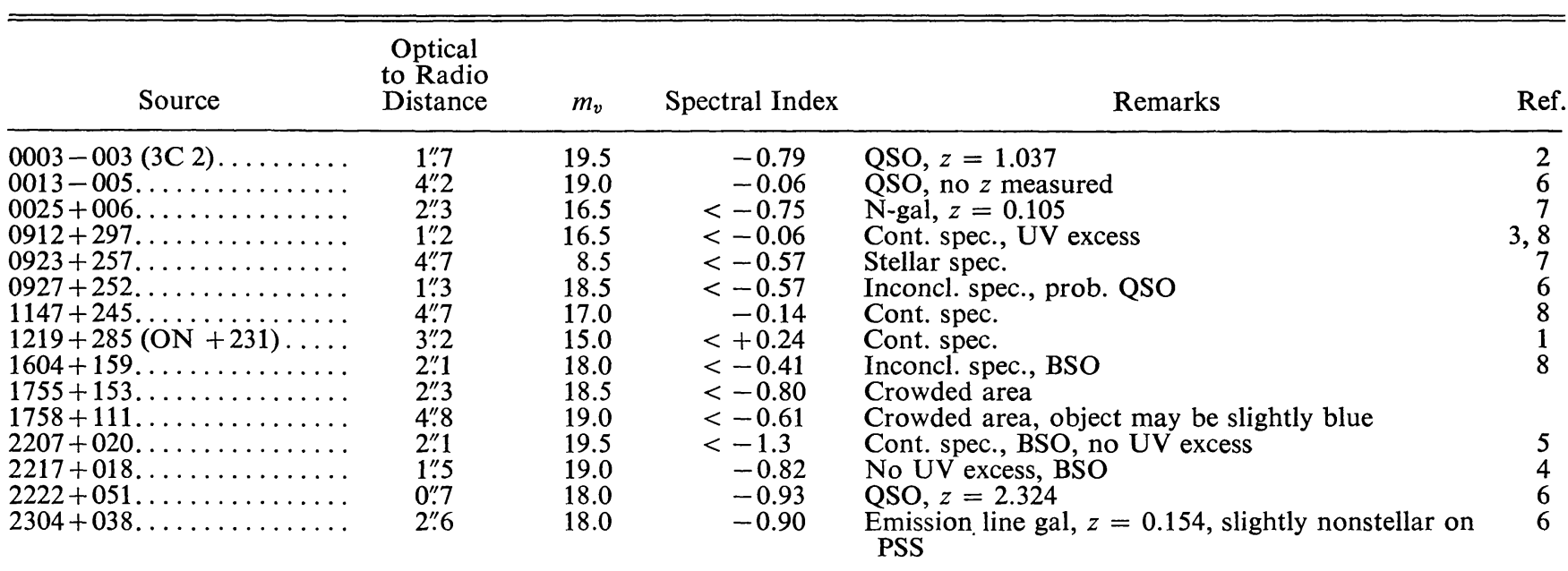

REFERENCES.-(1) Burbidge and Strittmatter 1972; Strittmatter et al. 1972; (2) Lynds 1967; (3) Fanti et al. 1975; (4) McEwan, Browne, and Crowther 1975; (5) Strittmatter et al. 1974; (6) D. Wills, private communications; (7) Wills and Wills 1974; (8) Wills and Wills 1976.

\section{REFERENCES}

Adams, T. F. 1977, Ap. J. Suppl., 33, 19.

Adgie, R. L. 1974, Observatory, 94, 300.

Agnew, D., and Arp, H. C. 1973, Pub. A.S.P., 85, 162.

Aizu, K. 1966, Pub. Astr. Soc. Japan, 8, 219.

Argue, A. N., and Taylor, C. M. 1974, Observatory, 94, 295.

Baldwin, J. A., Burbidge, E. M., Hazard, C., Murdoch, H. S. Robinson, L. B., and Wampler, E. J. 1973, Ap. J., 185, 739.

Bash, F. N., Cotton, W. D., and Douglas, J. N. 1974, A.J., 79, 1341.

Bergamini, R., Braccesi, A., Colla, G., Fanti, C., Fanti, R., Ficarra, A., Formiggini, L., Gandolfi, E., Gioia, I., Lari, C., Marano, B., Padrielli, L., Tomasi, P., and Vigotti, M. 1973, Astr. Ap., 23, 195.

Bolton, J. G., and Ekers, J. 1967, Australian J. Phys., 20, 109. Bolton, J. G., Shimmins, A. J., and Merkelijn, J. 1968 , Australian J. Phys., 21, 81.

Bolton, J. G., and Wall, J. V. 1970, Australian J. Phys., 23, 789.

Braccesi, A., Ficarra, A., Formiggini, L., Gandolfi, E., Lari, C., Padrielli, L., and Tomasi,'P. 1970, Astr. Ap., 6, 268.

Bridle, A. H., Davis, M. M., Fomalont, E. B., and Lequeux, J. 1972, A.J., 77, 405

Brown, G. S. 1973, Ph.D. thesis, University of Texas at Austin.

Browne, I. W. A., Crowther, J. H., and Adgie, R. L. 1973, Nature, 244, 146.

Brundage, R. K., Dixon, R. S., Ehman, J. R., and Kraus, J. D. 1971, A.J., 76, 777.

Burbidge, E. M., and Strittmatter, P. A. 1972, Ap. J. (Letters), 174, L57.

Caswell, J. L., and Wills, D. 1967, M.N.R.A.S., 135, 231.

Clarke, M. E., Bolton, J. G., and Shimmins, A. J. 1966, Australian J. Phys., 19, 375.

Colla, G., Fanti, C., Fanti, R., Gioia, I., Lari, C., Lequeux, J., Lucas, R., and Ulrich, M. H. 1975, Astr. Ap. Suppl., 20, 1. Condon, J. J., Balonek, T. J., and Jauncey, D. L. 1975, A.J., $80,887$.

Cotton, W. D. 1974, UTRAO Internal Report 44, November 15.

. 1976, Ap. J. Suppl., 32, 467.

Cotton, W. D., Owen, F. N., and Ghigo, F. D. 1975, A.J., 80, 353.

de Ruiter, H. R., Willis, A. G., and Arp, H. C. 1977, Astr. Ap. Suppl., 28, 486.
Douglas, J. N., Bash, F. N., Ghigo, F. D., Moseley, G. F., and Torrence, G. W. 1973, A.J., 78, 1.

Elsmore, B., and Mackay, C. D. 1969, M.N.R.A.S., 146, 361.

Fanti, C., Fanti, R., Ficarra, A., Formiggini, L., Giovanni, G., Lari, C., and Padrielli, L. 1975, Astr. Ap. Suppl., 19, 143.

Fomalont, E. B. 1971, A.J., 76, 513.

Fomalont, E. B., and Moffet, A. T. 1971, A.J., 76, 5.

Gearhart, M. R., Lund, J. M., Frantz, D. J., and Kraus, J. D. 1972, A.J., 77, 557.

Ghigo, F. D., and Owen, F. N. 1973, A.J., 78, 848.

Grueff, G., and Vigotti, M. 1972, Astr. Ap. Suppl., 6, 1.

$$
\text { 1973, Astr. Ap. Suppl., 11, } 41 .
$$$$
\text { 1975, Astr. Ap., 20, } 57
$$

Hazard, C., Gulkis, S., and Bray, A. D. 1967, Ap. J., 148, 669.

Hazard, C., and Jauncey, D. L. 1972, A.J., 77, 621.

Hazard, C., Jauncey, D. L., and Backer, D. C. 1970, A.J., 75 , 1039.

Hunstead, R. W. 1971, M.N.R.A.S., 152, 277.

Jauncey, D. L., and Hazard, C. 1970, Ap. Letters, 7, 1.

Jauncey, D. L., and Niell, A. E. 1971, Nature Phys. Sci., 229, 223.

Johnson, K. H. 1974, A.J., 79, 1006.

Kristian, J., and Sandage, A. 1970, Ap. J., 162, 391.

Kristian, J., Sandage, A., and Katem, B. 1974, Ap. J., 191, 43.

Lynds, R. 1967, Ap. J., 147, 837.

Lynds, R., and Wills, D. 1972, Ap. J., 172, 531

Macdonald, G. H., Kenderdine, S., and Neville, A. C. 1968 , M.N.R.A.S., 138, 259.

Macdonald, G. H., and Miley, G. K. 1971, Ap. J., 164, 237.

Mackay, C. D. 1969, M.N.R.A.S., 145, 31.

Marsden, B. G. 1976 a IAU Circ., No. 2939.

1976b, IAU Circ., No. 2954.

McEwan, J. J., Browne, I. W. A., and Crowther, J. H. 1975, Mem. R.A.S., 80, 1.

Merkelijn, J. K. 1969, Australian J. Phys., 22, 237.

Merkelijn, J. K., and Wall, J. V. 1970, Australian J. Phys., 23, 575.

Olsen, E. T. 1970, A.J., 75, 764.

Radivich, M. M., and Kraus, J. D. 1971, A.J., 76, 683.

Riley, J. M., and Pooley, G. G. 1975, Mem. R.A.S., 80, 105.

Rinsland, C. P., Dixon, R. S., and Kraus, J. D. 1975, A.J., 80, 759.

Schmidt, M. 1972, Ap. J., 176, 273. 
Schmidt, M. 1974, Ap. J., 193, 505.

Sharp, J. R., and Bash, F. N. 1975, A.J., 80, 335.

Shimmins, A. J., Bolton, J. G., and Wall, J. V. 1975, Australian J. Phys. Ap. Suppl., 34, 63.

Strittmatter, P. A., Carswell, R. F., Gilbert, G., and Burbidge, E. M. 1974, Ap. J., 190, 509 .

Strittmatter, P. A., Serkowski, K., Carswell, R., Stein, W. A., Merrill, K. M., and Burbidge, E. M. 1972, Ap.J. (Letters), 175, L7.

Stull, M. A. 1973, A.J., 78, 285.

Véron, M. P. 1972, Astr. Ap., 20, 471.

Véron, M. P., and Véron, P. 1974, Astr. Ap. Suppl., 18, 309. 1975, Astr. Ap., 42, 1.
Webster, A. 1976, M.N.R.A.S., 175, 71.

Wills, B. J. 1973, Ap. J., 180, 335.

- 1976, A.J., 81, 1031

Wills, B. J., Wills, D., and Douglas, J. N. 1973, A.J., 78, 521.

Wills, D., and Bolton, J. G. 1969, Australian J. Phys., 22, 775.

Wills, D., and Wills, B. J. 1974, Ap. J., 190, 271. .1976, Ap. J. Suppl., 31, 143.

Willson, M. A. G. 1972, M.N.R.A.S., 156, 7.

Wyndham, J. D. 1966, Ap. J., 144, 459.

Zwicky, F., Herzog, E., Wild, P., Karpowicz, M., and Kowal, C. T. 1961-1968, Catalog of Galaxies and Clusters of Galaxies (in 6 vols.; Pasadena: California Institute of Technology Press).

Frank D. Ghigo: Physics Department, Brandeis University, Waltham, MA 02154 


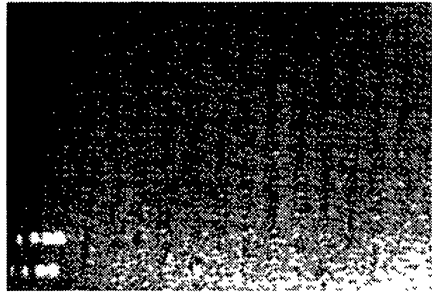

0033-000 B

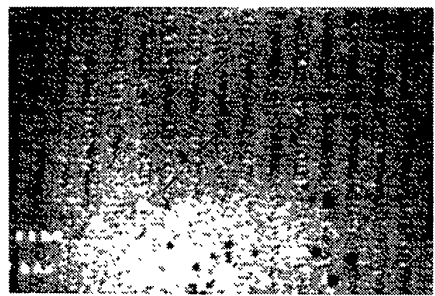

$0103+062 R$
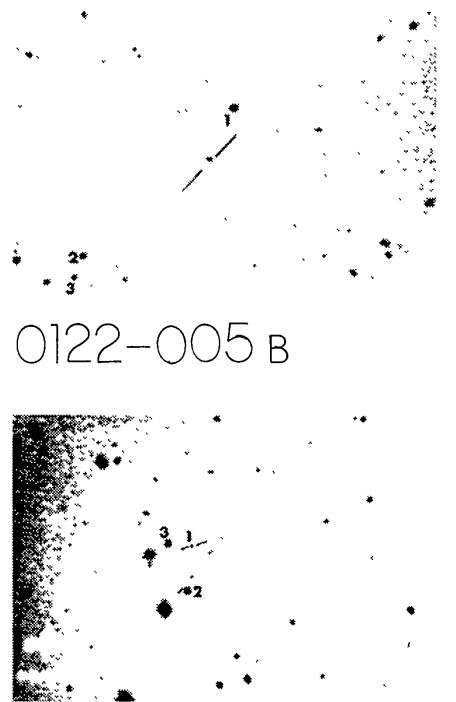

$0828+324$ B

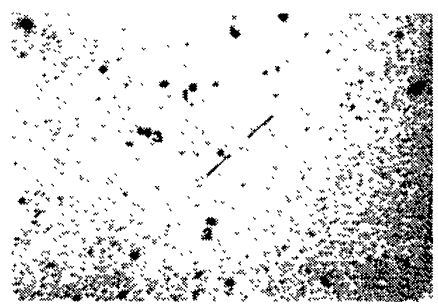

$0845+298 R$

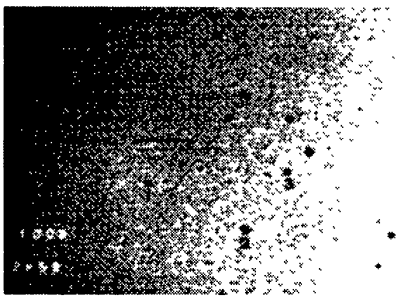

$0038+097 R$

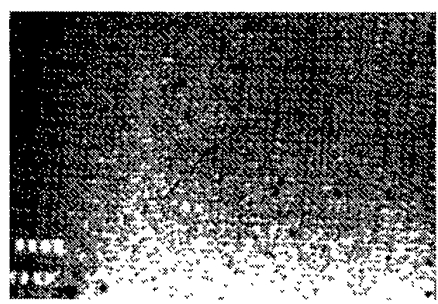

$0103+089 R$

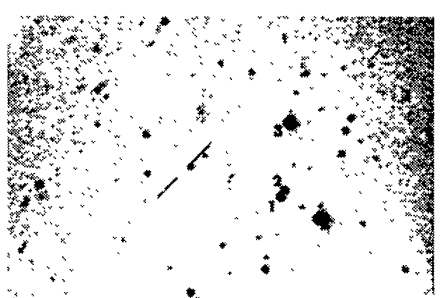

$0807+276$ B

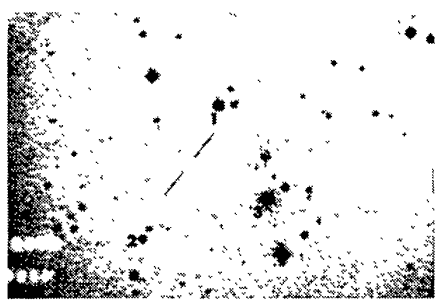

$0828+325 R$

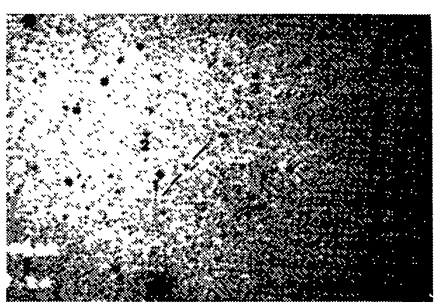

$0903+258 R$

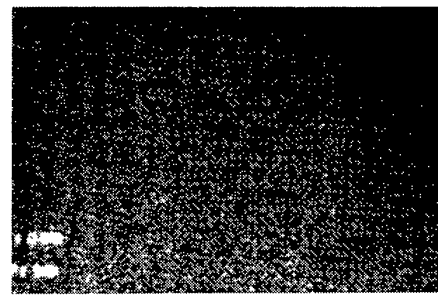

$0100+050$ в

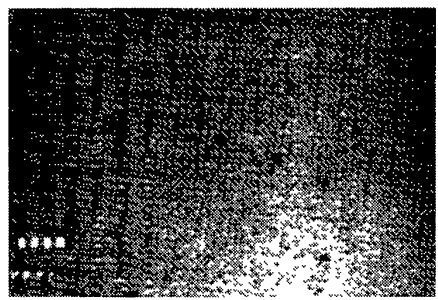

$0119-005 \mathrm{R}$

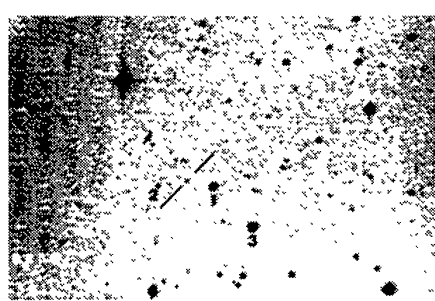

$0818+295 R$

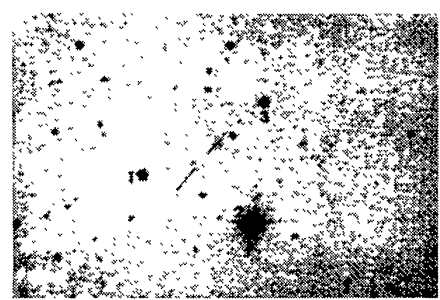

$0832+267 \mathrm{R}$

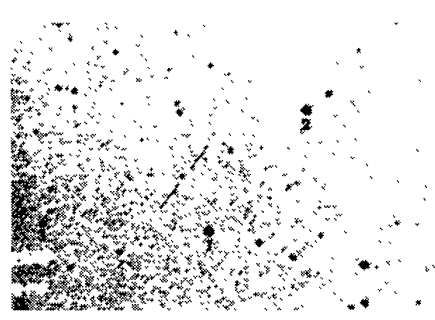

$0910+238 R$

FIG. 2.-Identification charts for 118 new and revised identifications, reproduced, with permission, from the National Geographic Society-Palomar Observatory Sky Survey plates. The reticle lines indicate the radio position, not necessarily the identification.

GHIGO (see page 384) 


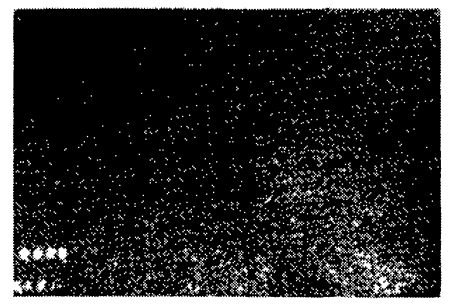

$0912+253 R$

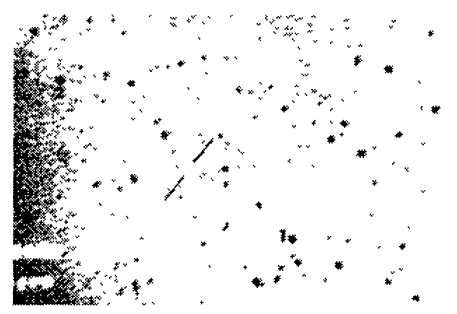

$0919+313$ B

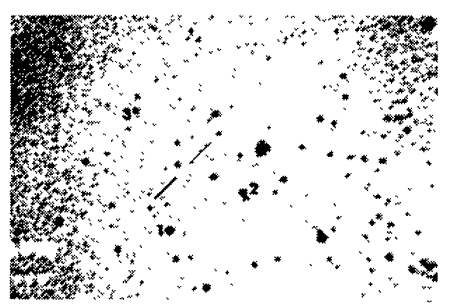

$0932+241 B R$
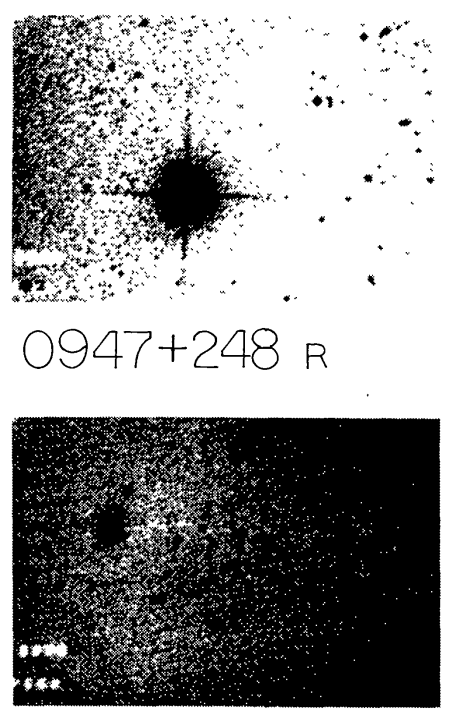

$1032+265 R$

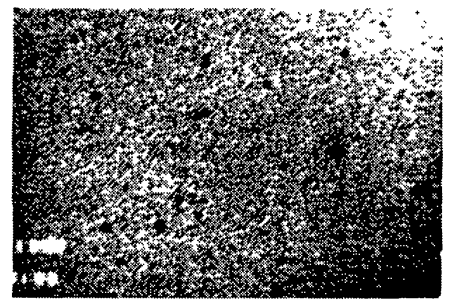

$0914+257 R$

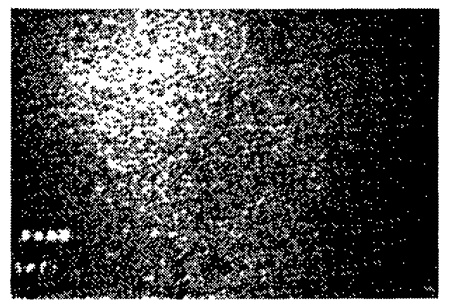

$0927+252$ B

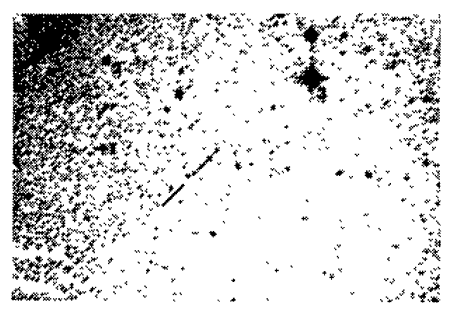

$0936+247 R$
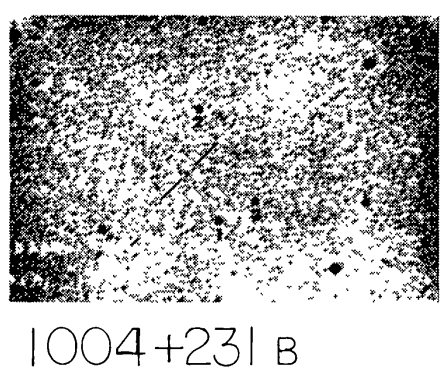

E

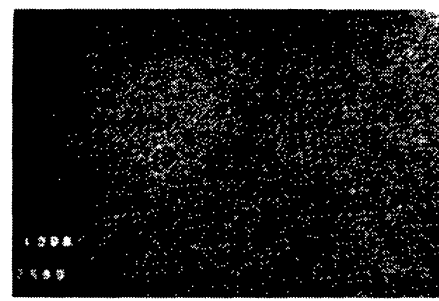

$1032+260 B$

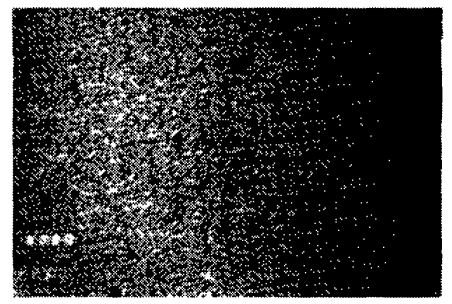

$0916+263$ B

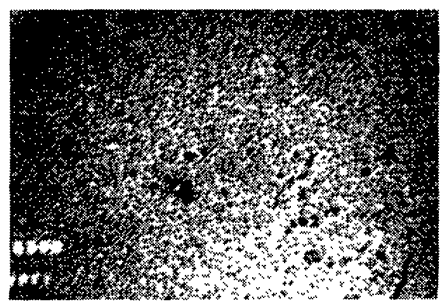

$0932+241$ A R

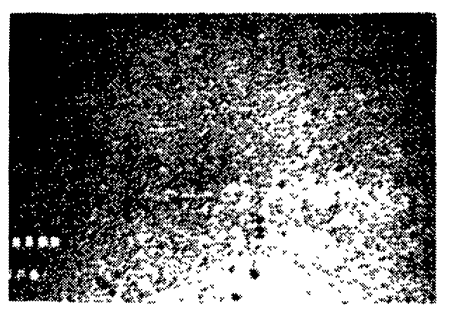

$0942+276 R$

FIG. 2.-Continued

GHigo (see page 384 ) 


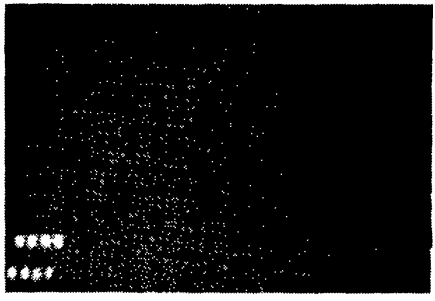

$1106+252 R$

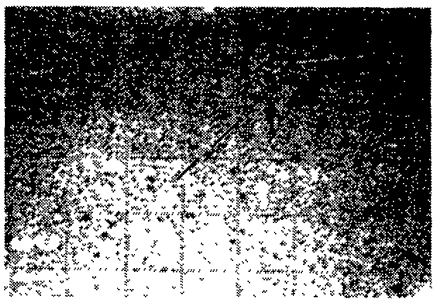

$1207+118 \mathrm{~B}$

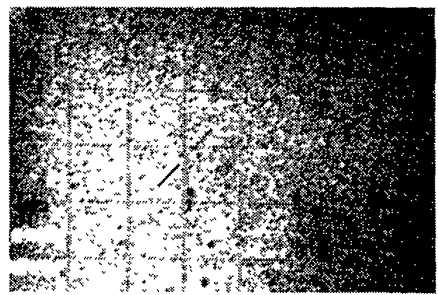

$1234+252 R$

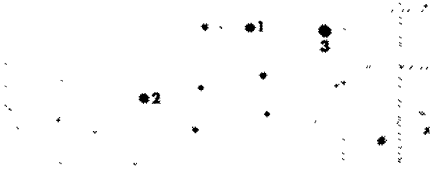

$1239+168 R$

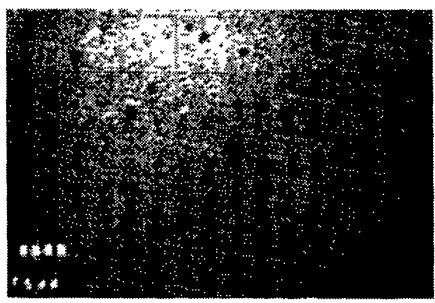

$1303+192 R$

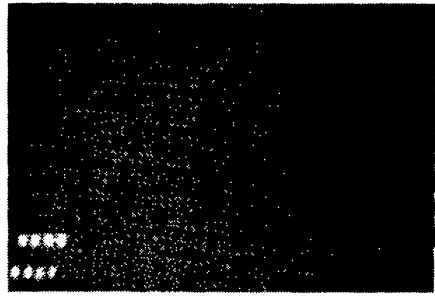

$1118+237 R$

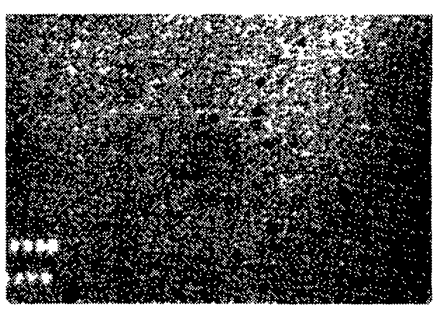

$1222+264 R$

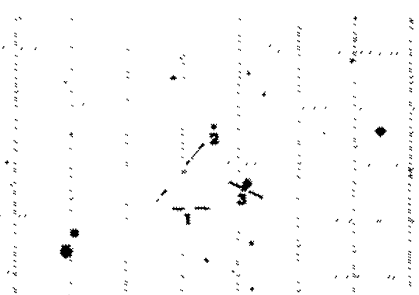

$1235+196 B$

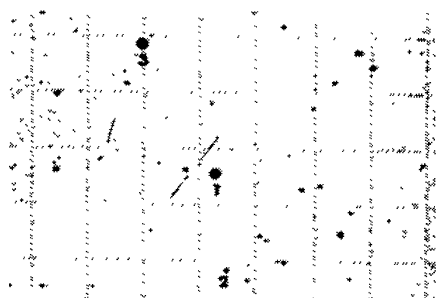

, N

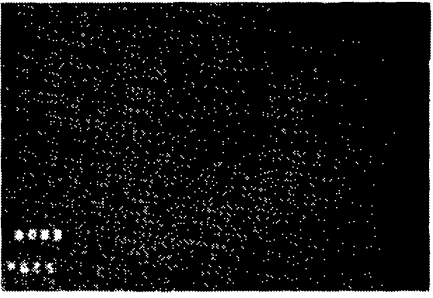

$1136+299 R$

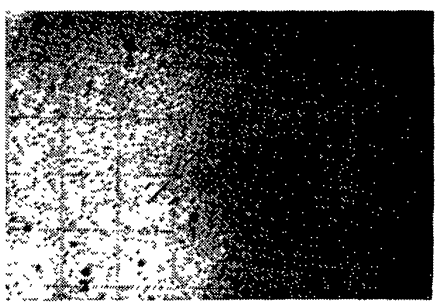

$1228+256$ B

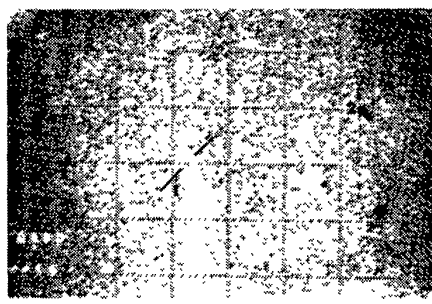

$1236+327 \mathrm{~B}$

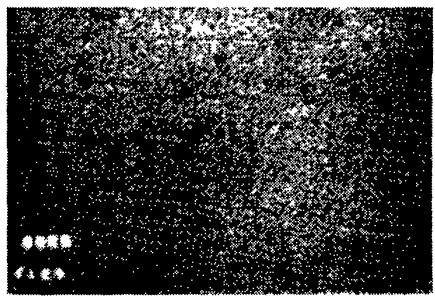

$1300+320 R$

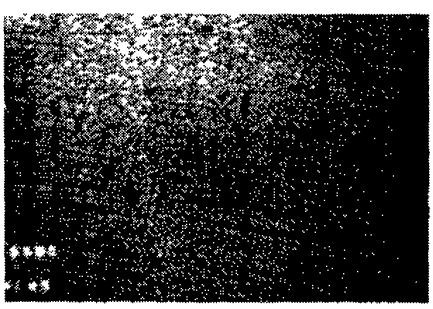

$1304+243 R$

FIG. 2.-Continued

GHIGo (see page 384 ) 

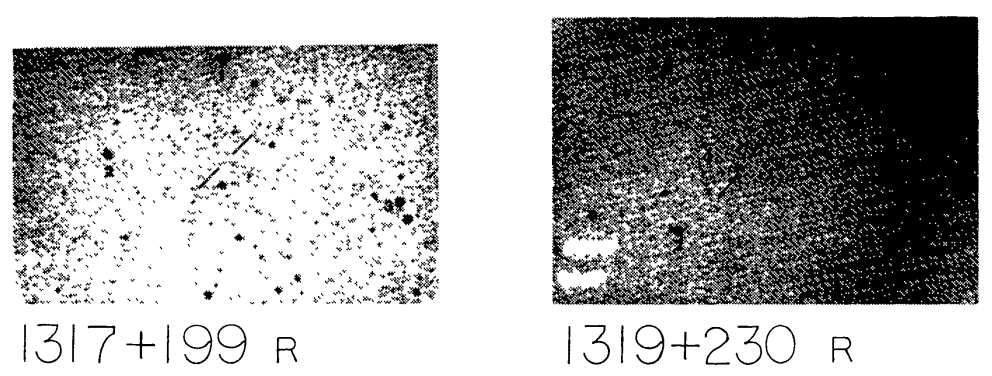

$1319+230 R$

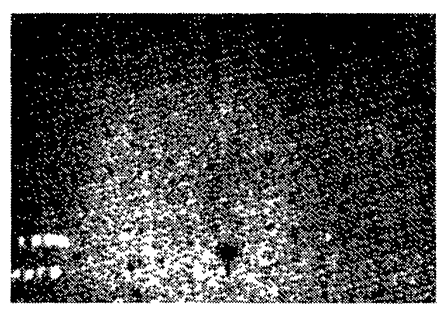

$1325+126 R$
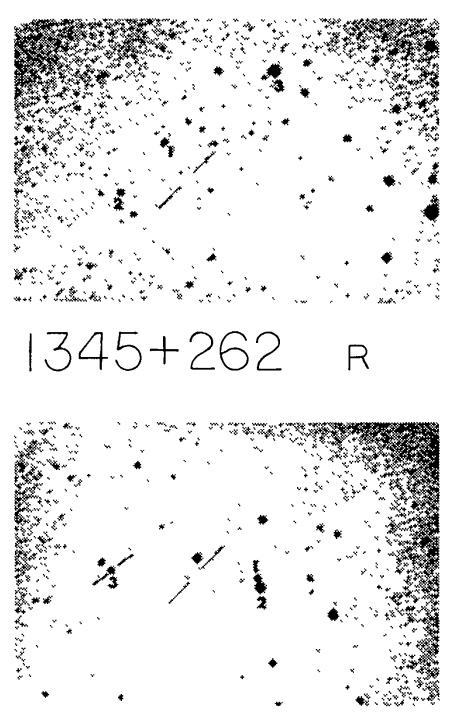

$1408+141 R$

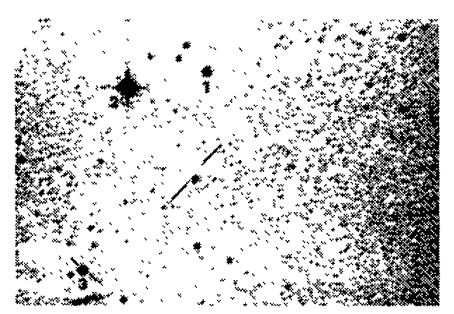

$1425+287 R$

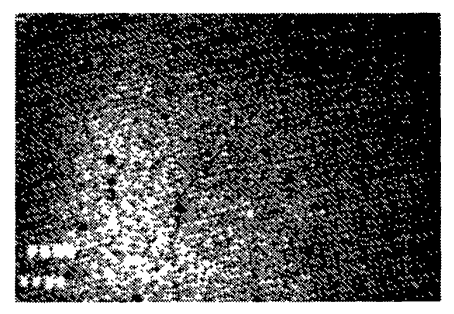

$1326+150 \mathrm{R}$

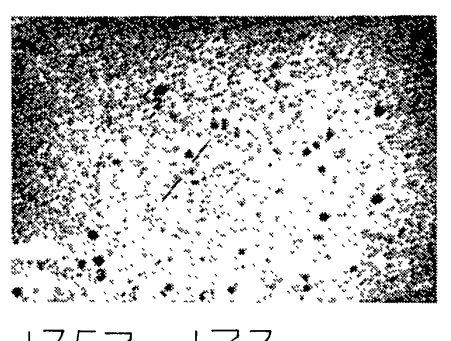

$1357+173 \quad R$

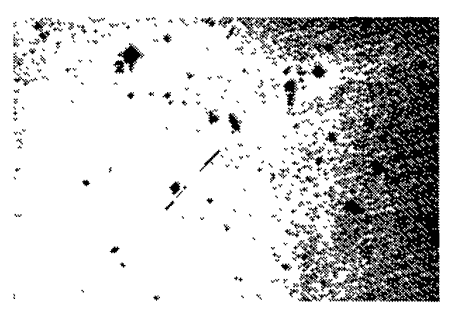

$$
1413+245 \quad R
$$

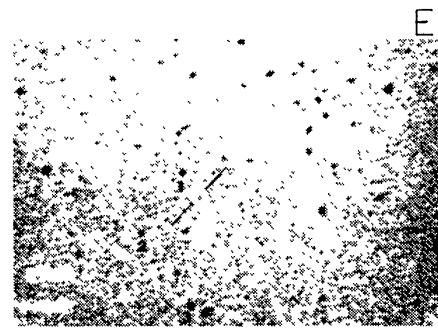

$1428+260 R$

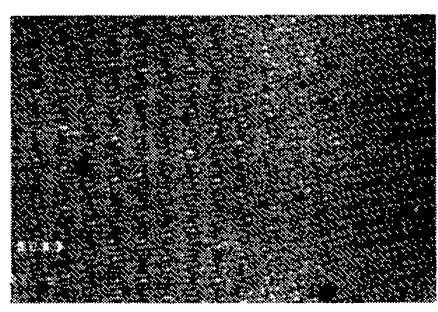

$1325+124 R$

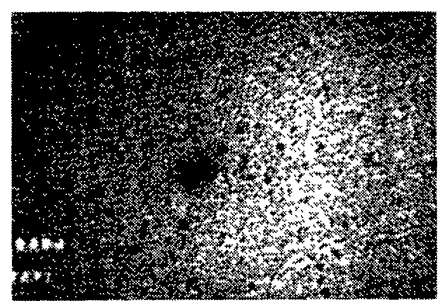

$1339+266$ B
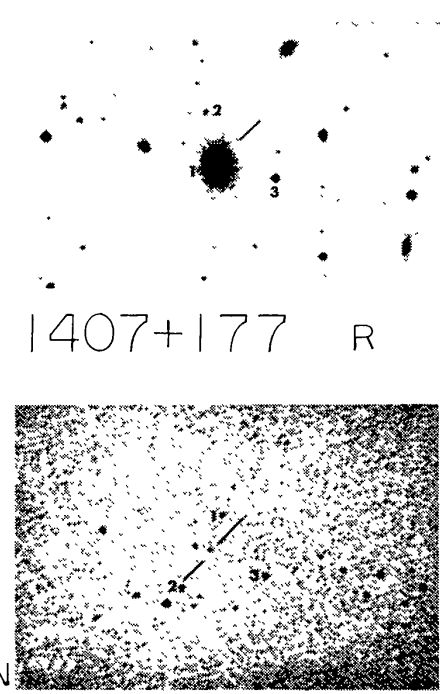
$2^{\prime}$ $1422+128$

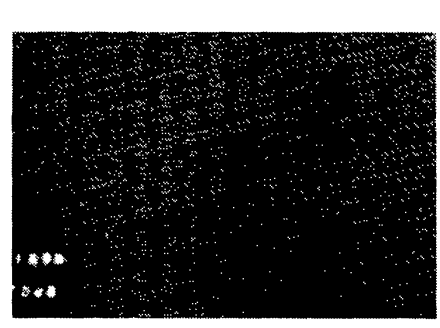

$1435+304 R$

FIG. 2.-Continued

GHIGo (see page 384) 
PLATE 33
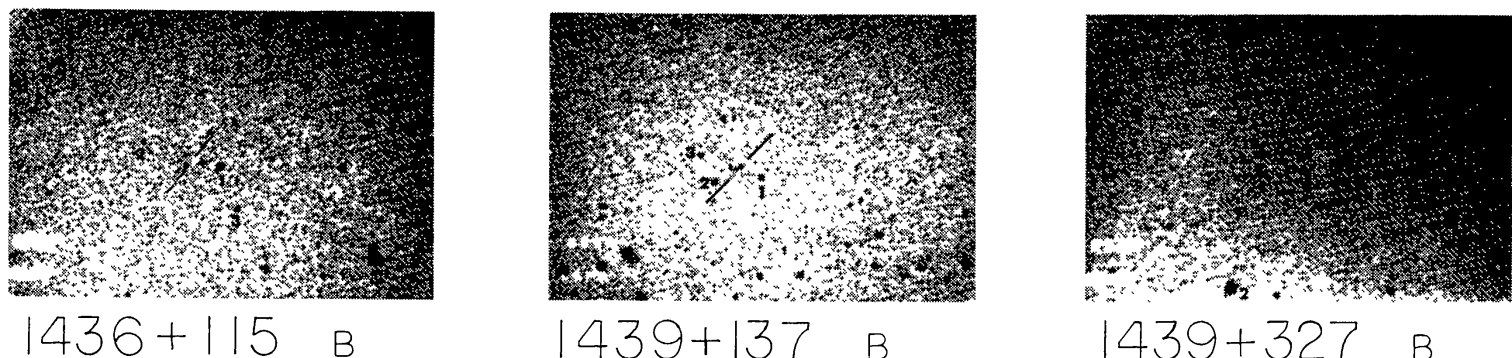
$1439+137$
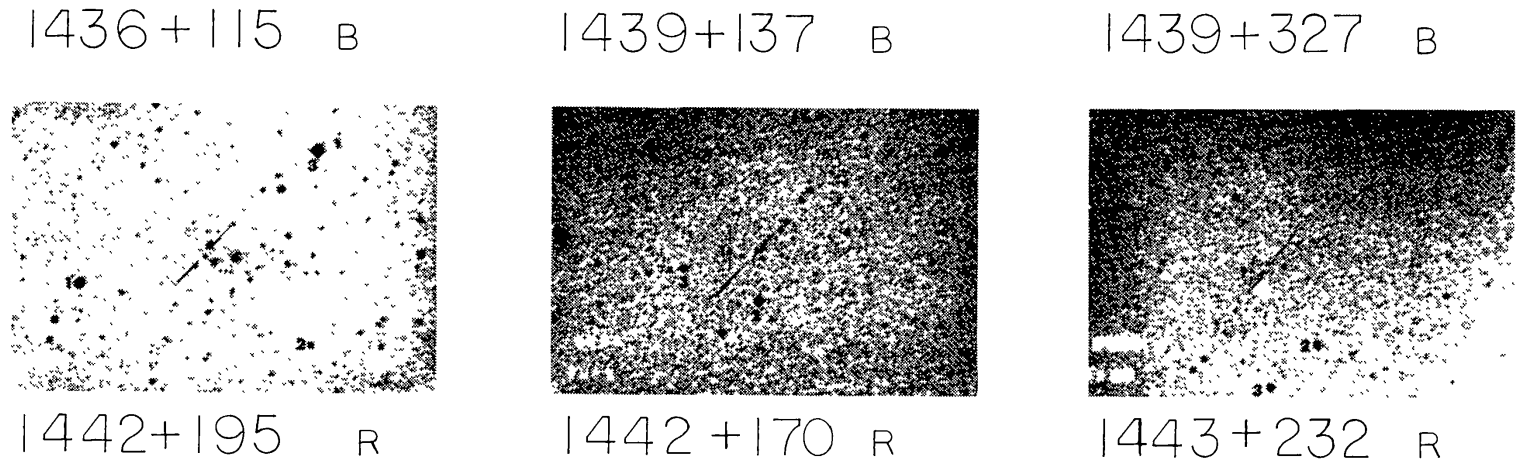

$1443+232 R$
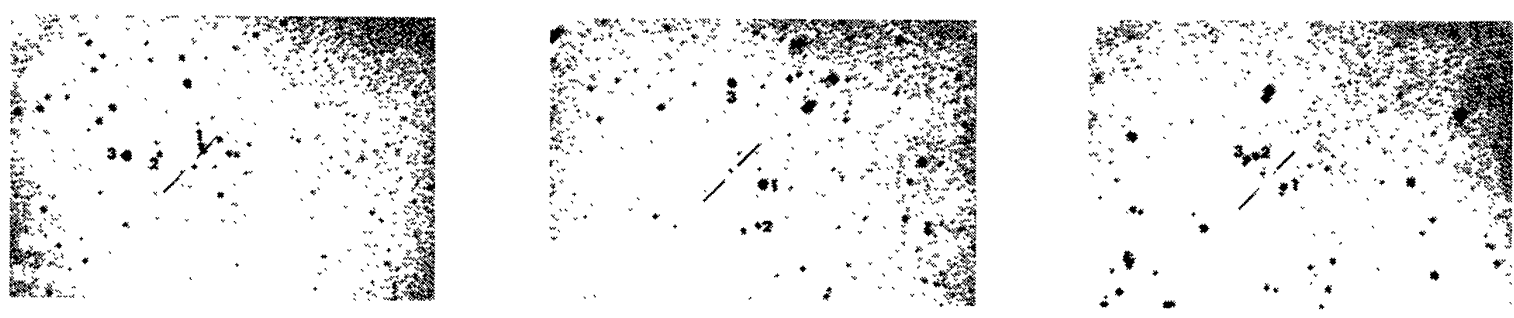

$1449+144 R$

$$
1452+258 \quad R
$$$$
1457+147 \quad R
$$
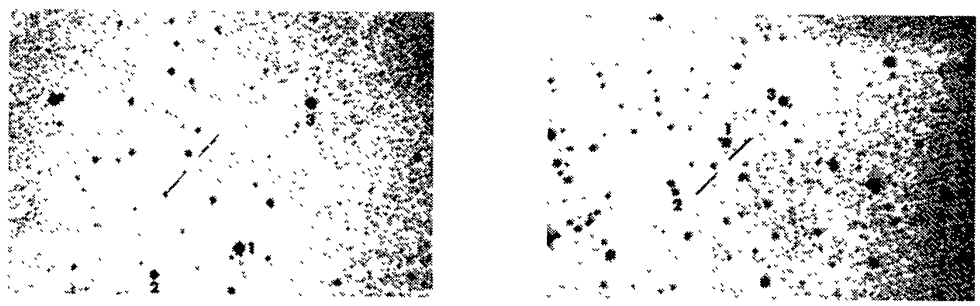

$1518+156 \quad R$

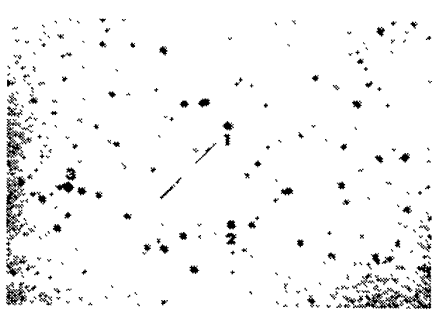

$1601+116 \mathrm{R}$
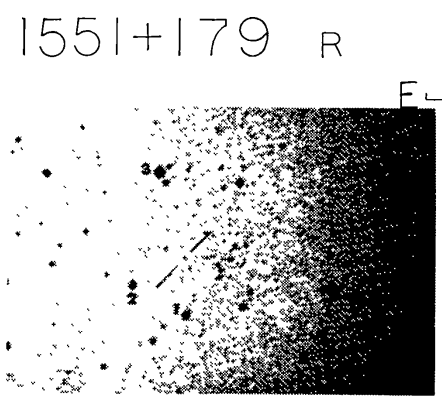

$1608+191 \mathrm{~B}$

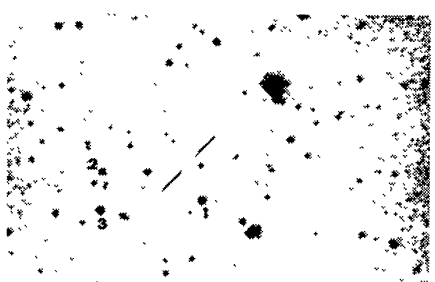

$2^{\prime} N$

$1559+157 R$

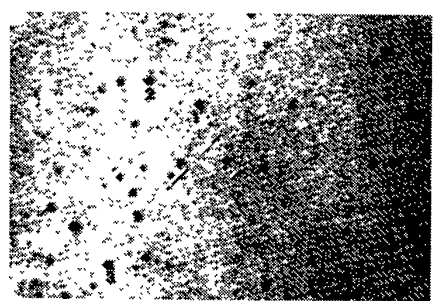

$1612+176 \quad$ R

FIG. 2.-Continued

GHIGO (see page 384) 


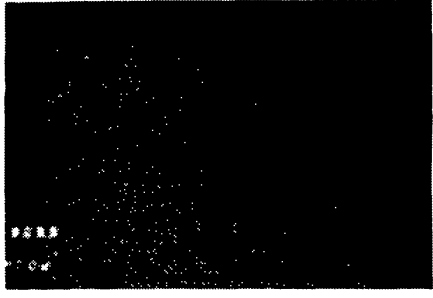

$1618+108 \quad B$

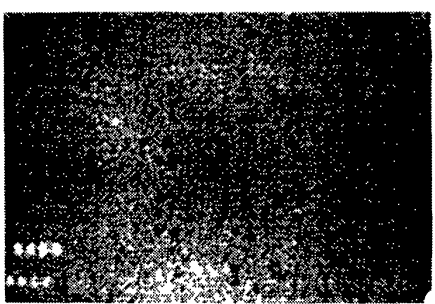

$1627+138 R$

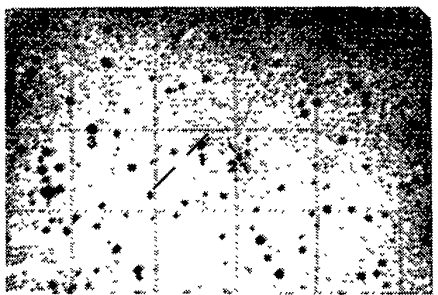

$1706+188 \quad R$
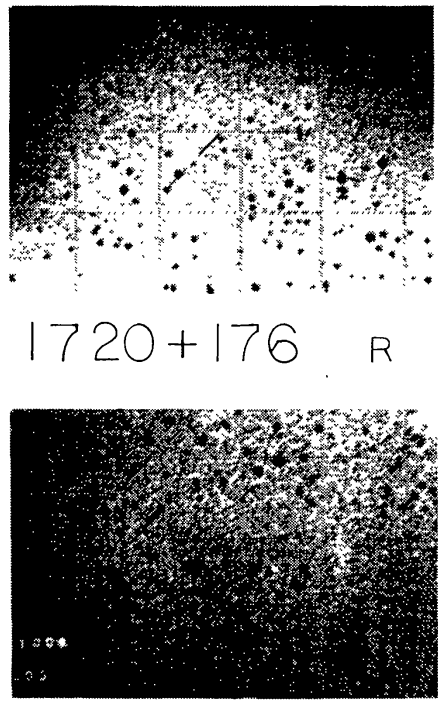

$1736+161 \quad R$

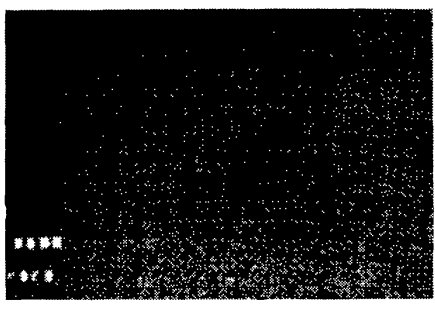

$1620+127 R$

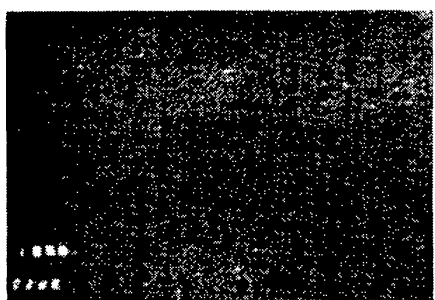

$1648+198 R$

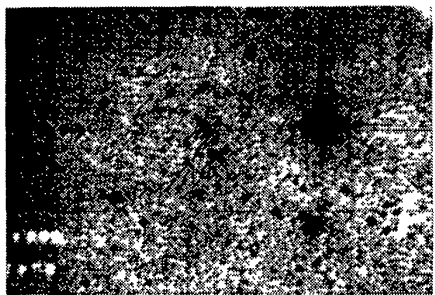

$1707+117 \quad R$

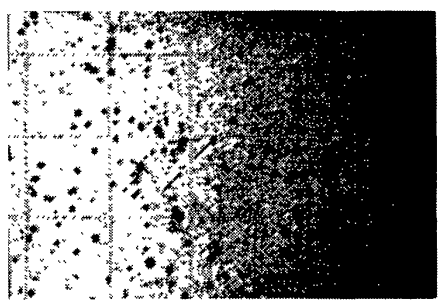

$1727+174 R$

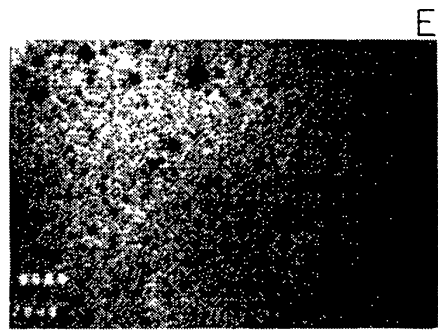

$1739+107 R$

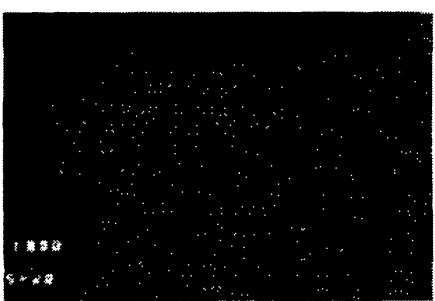

$1621+140 R$

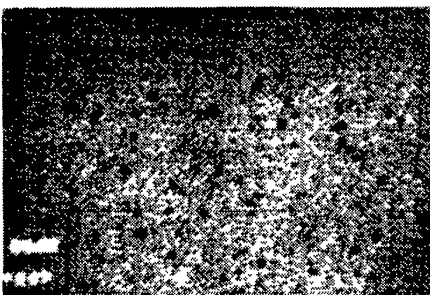

$1653+163 R$

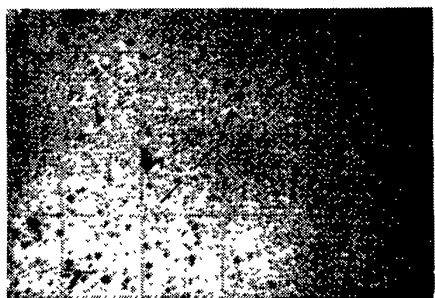

$1717+130 \quad R$

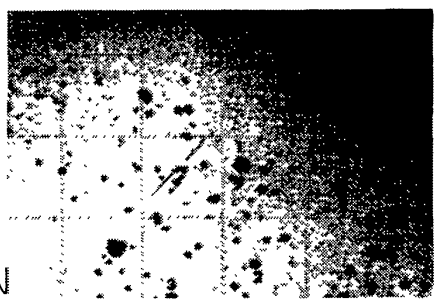

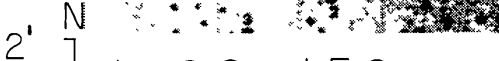

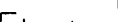

FIG. 2.-Continued

GHIGo (see page 384) 

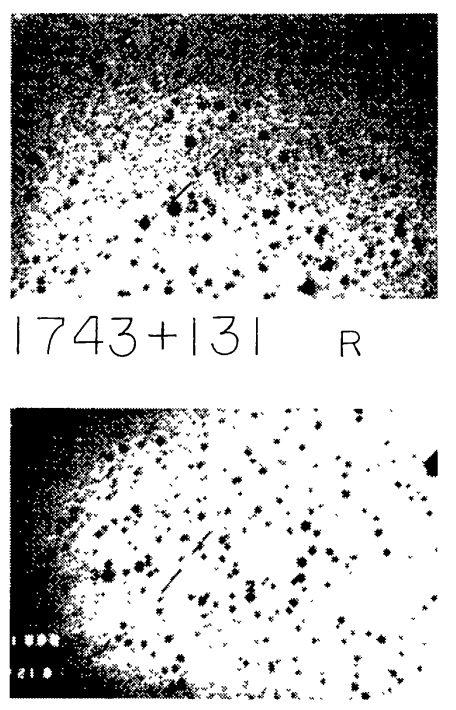

$175 \mid+153 \quad R$
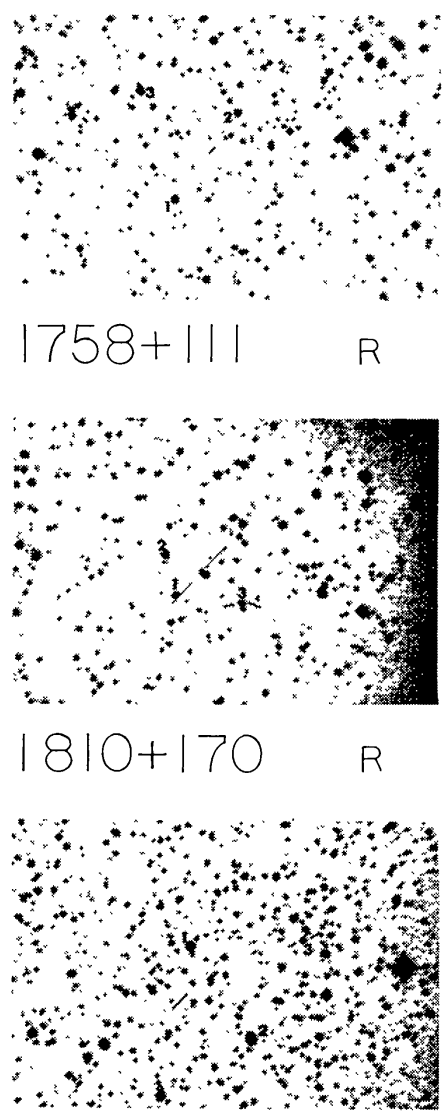

$1822+127 \quad B$
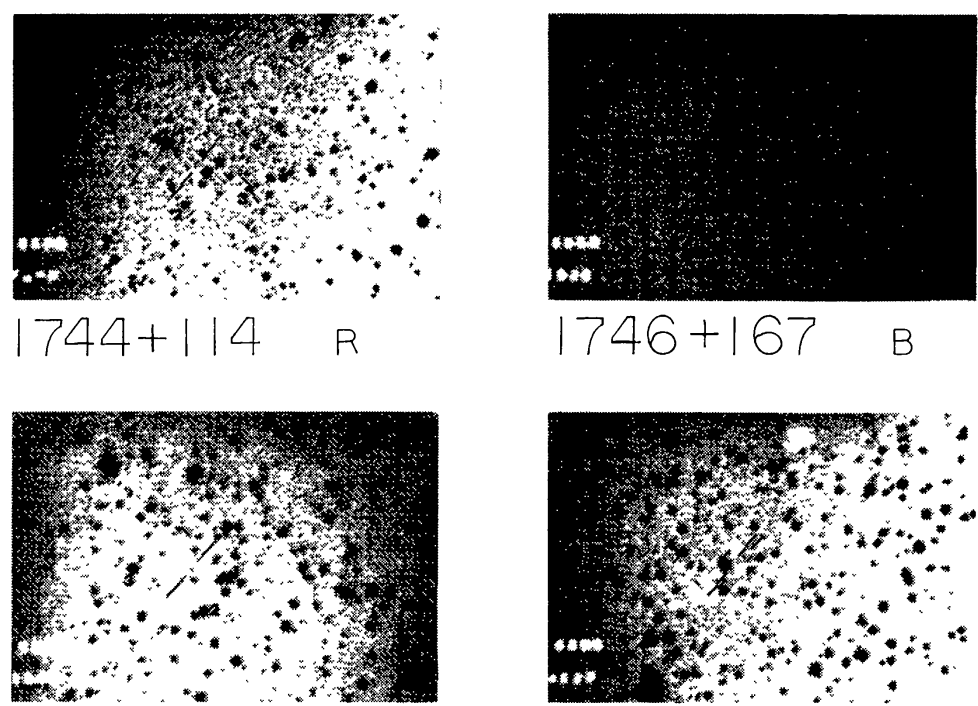

$1755+165 \quad R$

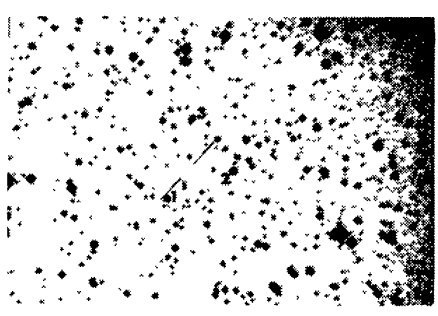

$1759+138$

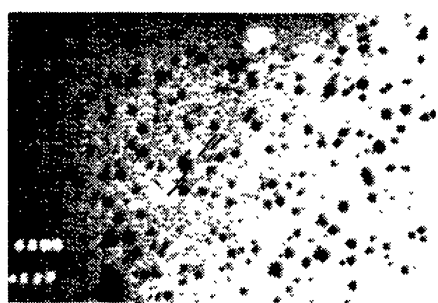

$1755+153 R$

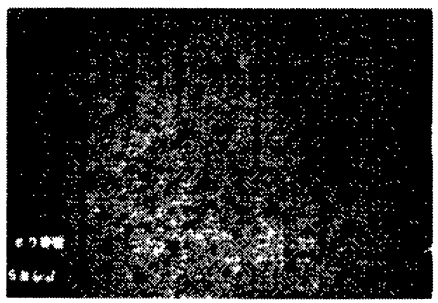

$1804+157 \quad R$

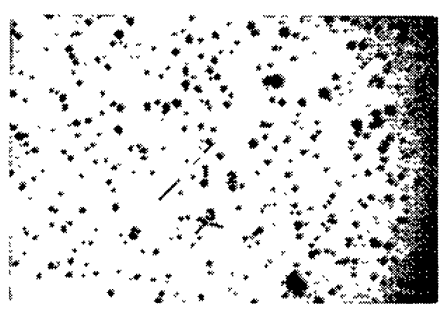

$1817+157 \quad B$

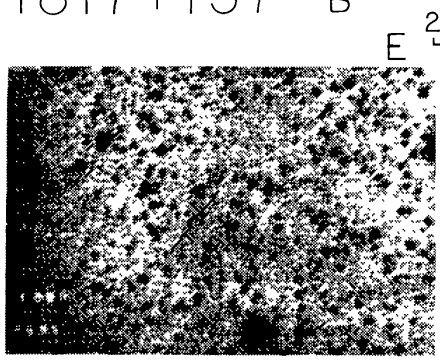

$1823+162 R$

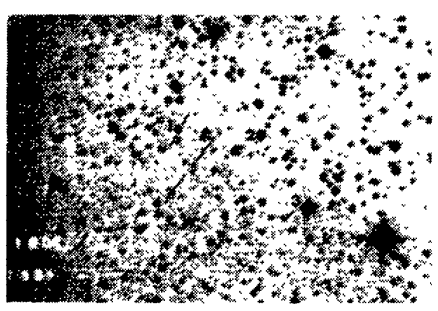

$1820+179 \quad R$

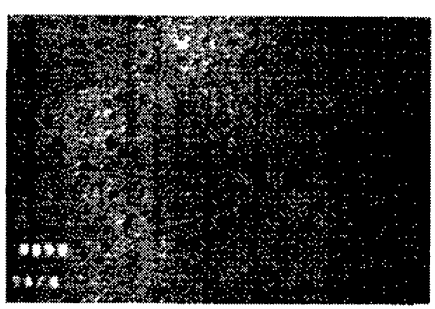

$2042+032 \quad R$

FIG. 2.-Continued

GHigo (see page 384) 


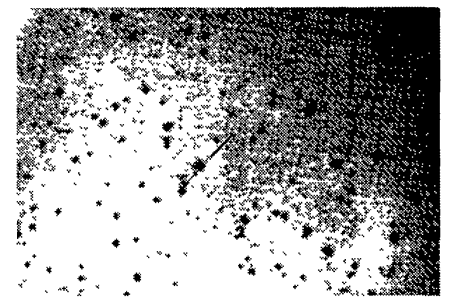

$2055+088 R$

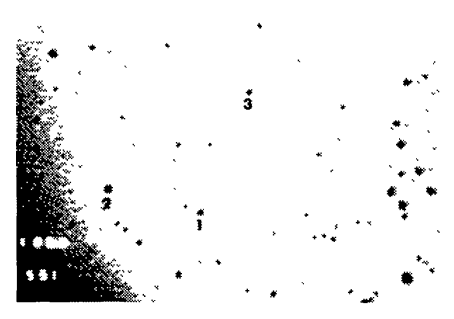

$2122+052 \quad R$

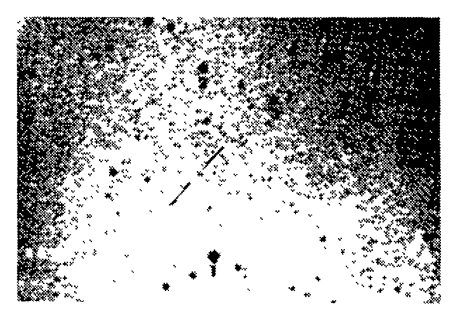

2225-019 R

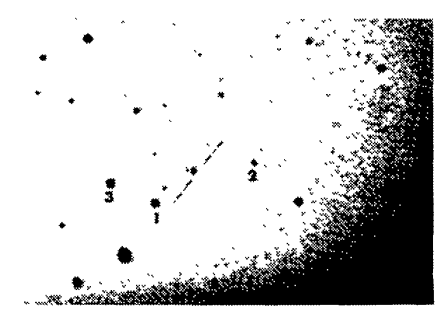

$2304+038 \quad B$

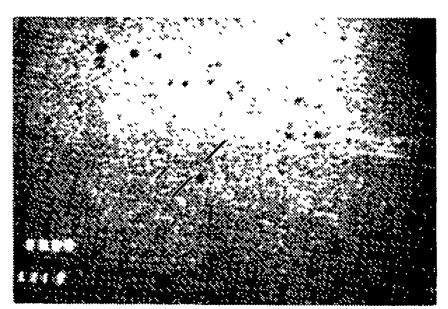

$2345+048 R$
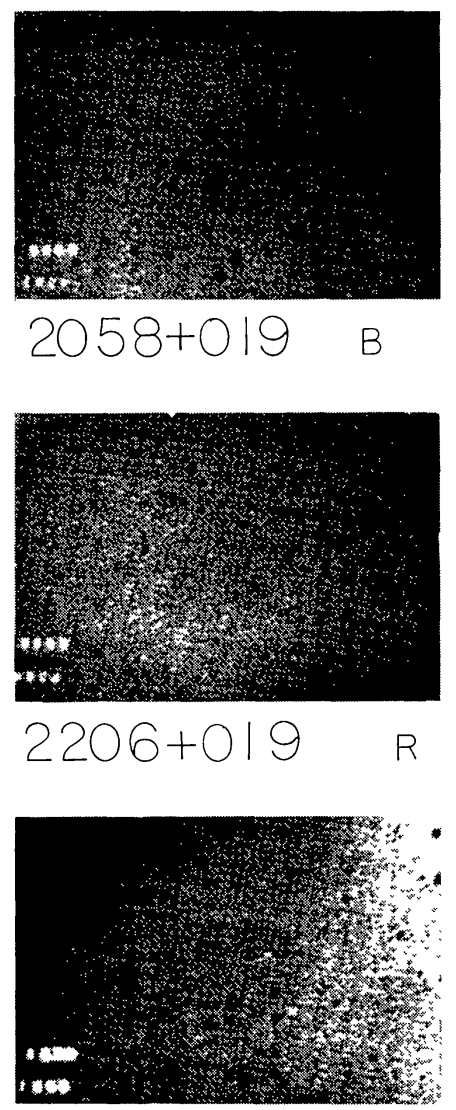

$2238+033 \quad R$

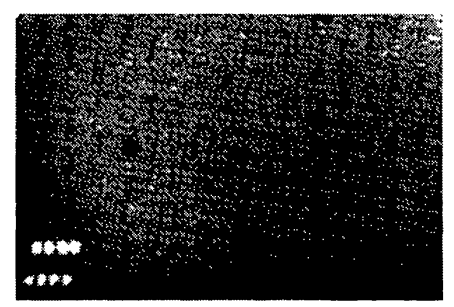

$2318+081 R$

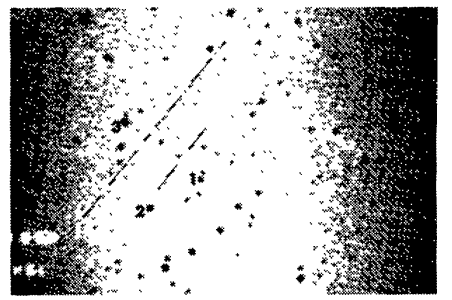

$2|2|+028 \quad B$

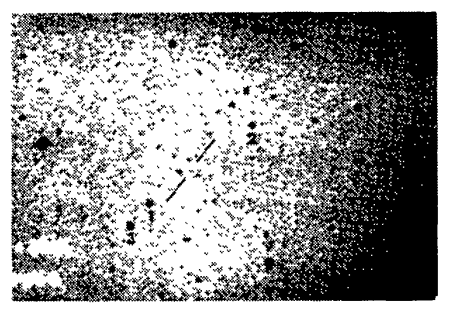

2215-000 R

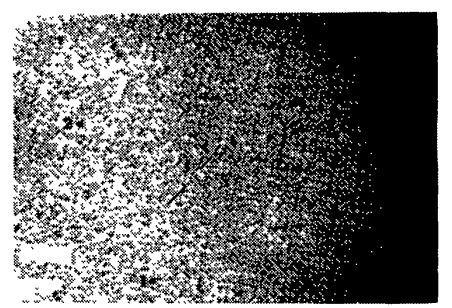

$2259+083 \quad R$

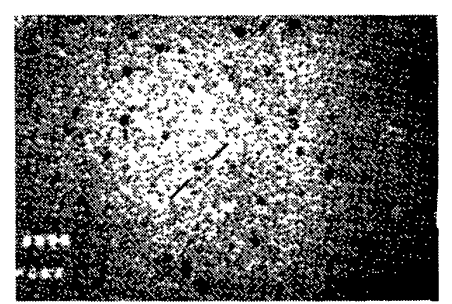

$2330+015 \quad R$

FIG. 2.--Continued

GHIGo (see page 384) 